\title{
Nickel-Catalyzed Carboxylation of Aryl and Heteroaryl Fluorosulfates Using Carbon Dioxide
}

\section{(Supporting Information)}

Cong Ma, ${ }^{\dagger}$ Chuan-Qi Zhao, ${ }^{\dagger}$ Xue-Tao Xu, ${ }^{\ddagger}$ Zhao-Ming Li, ${ }^{\dagger}$ Xiang-Yang Wang, ${ }^{\ddagger}$ Kun Zhang, ${ }^{\ddagger}$ Tian-Sheng Mei ${ }^{*} \dagger$

${ }^{\dagger}$ State Key Laboratory of Organometallic Chemistry, Center for Excellence in Molecular Synthesis, Shanghai Institute of Organic Chemistry, Chinese Academy of Sciences, 345 Lingling Lu, Shanghai 200032, China

${ }^{\ddagger}$ School of Biotechnology and Health Sciences, Wuyi University, Jiangmen 529020, China.

\section{Table of Contents}

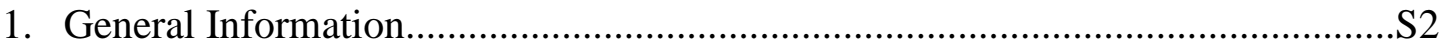

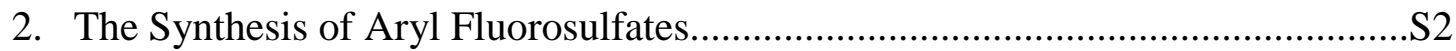

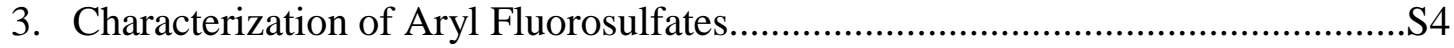

4. The Synthesis of Pyridinyl Fluorosulfates............................................................

5. Characterization of Pyridinyl Fluorosulfates.................................S11

6. General Procedure for the Carboxylation of Aryl Fluorosulfates................S13

7. Characterization Data for the Carboxylative Products of Aryl Fluorosulfates...S14

8. General Procedure for the Direct Carboxylation of Aryl Phenols...............S20

9. General Procedure for the Carboxylation of Pyridinyl Fluorosulfates..........S23

10. Characterization Data for the Carboxylative Products of Pyridinyl Fluorosulfates

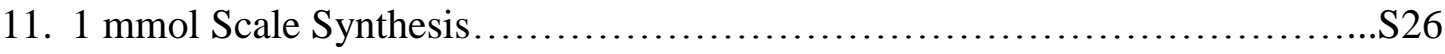

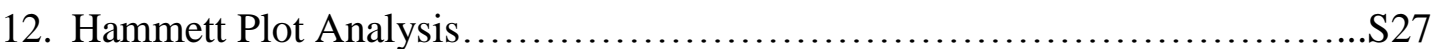

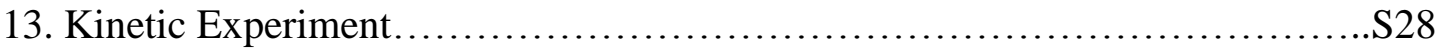

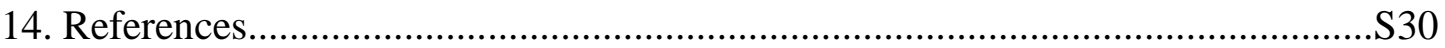

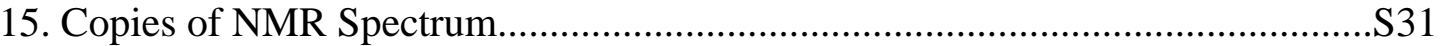




\section{General Information}

All commercial reagents were purchased from TCI, Sigma-Aldrich, Adamas-beta, Bide Pharmatech Ltd. of the highest purity grade. They were used without further purification unless specified. Solvents and commercially available reagents were used without purification. Column chromatography was performed using either 100-200 Mesh or 300-400 Mesh silica gel. Visualization of spots on TLC plate was accomplished with UV light (254 nm) and staining over $\mathrm{I}_{2}$ chamber. ${ }^{1} \mathrm{H}$ NMR and ${ }^{13} \mathrm{C}$ NMR spectra were recorded on Agilent AV 400, Varian Inova 400 (400 MHz and 100 $\mathrm{MHz}$, respectively). The peaks were internally referenced to TMS $(0.00 \mathrm{ppm})$ or residual undeuterated solvent signal. The following abbreviations were used to explain multiplicities: $\mathrm{s}=$ singlet, $\mathrm{d}=$ doublet, $\mathrm{t}=$ triplet, $\mathrm{q}=$ quartet, $\mathrm{m}=$ multiplet, and $\mathrm{br}=$ broad. High resolution mass spectra were recorded at the Center for Mass Spectrometry, Shanghai Institute of Organic Chemistry. Analytical and spectral data of all those known compounds are exactly matching with the reported values.

\section{The Synthesis of Aryl Fluorosulfates}

\subsection{Scope of aryl fluorosulfates}


<smiles>CC(C)(C)c1ccc(OC(F)(F)F)cc1</smiles>

1a<smiles>Cc1ccc(OC(F)(F)F)cc1</smiles>

$1 e$<smiles>Fc1cccc(O[S-])c1</smiles>

$1 i$<smiles>CC(=O)c1ccc(OC(F)(F)F)cc1</smiles>

$1 \mathrm{~m}$<smiles>CS(=O)(=O)c1ccc(OC(F)(F)F)cc1</smiles>

$1 q$<smiles>CCOC(=O)c1c(C)n(C)c2ccc(OS(F)(F)F)cc12</smiles><smiles></smiles>

1b<smiles>Cc1cc(C)cc(OC(F)(F)F)c1</smiles>

1f<smiles>Fc1cc(F)cc(O[S-](F)(F)F)c1</smiles>

1j<smiles>FC(F)(F)Oc1ccc(C(F)(F)F)cc1</smiles>

$1 \mathrm{n}$<smiles>FC(F)(F)Oc1ccc2ccccc2c1</smiles>

$1 \mathrm{r}$<smiles>Cc1ccccc1OC(F)(F)F</smiles>

1v<smiles></smiles>

$1 c$<smiles>[O-]c1ccccc1</smiles>

19<smiles>[O-]c1ccc(Cl)cc1</smiles>

$1 k$<smiles>N#Cc1ccc(OC(F)(F)F)cc1</smiles>

10<smiles>O=C1CCCc2cc(OC(F)(F)F)ccc21</smiles>

$1 \mathrm{~s}$<smiles>O=c1c2ccccc2oc2cc(OC(F)(F)F)ccc12</smiles><smiles>Fc1ccccc1OC(F)(F)F</smiles><smiles>[X]c1ccccc1-c1ccccc1</smiles><smiles>CCC(=O)OC1CCC2[C@@H]3CCc4cc([O-])ccc4[C@H]3CC[C@]12C</smiles>

$1 y$

\subsection{General Procedure I for the Synthesis of Aryl Fluorosulfates}

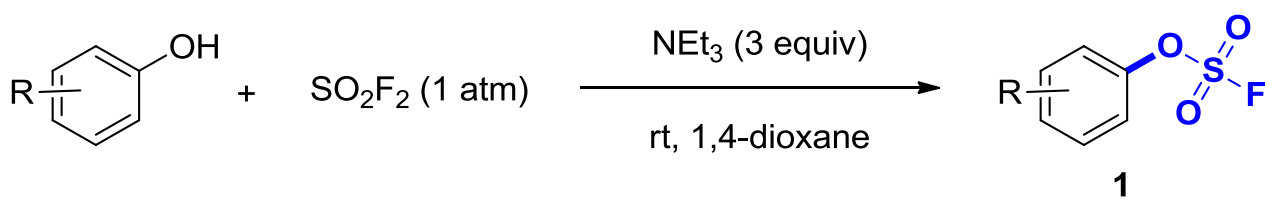

All the aryl fluorosulfates were synthesized according to literature ${ }^{1}$. To a $100 \mathrm{~mL}$ round bottom flask with a stir bar was added the corresponding phenol (1.0 equiv), $\mathrm{NEt}_{3}$ (3 equiv) and 1,4-dioxane $(50 \mathrm{~mL})$. The flask was capped with a rubber septum. 
Then $\mathrm{SO}_{2} \mathrm{~F}_{2}$ gas was introduced into the stirring reaction mixture by slow bubbling through a $\mathrm{SO}_{2} \mathrm{~F}_{2}$ balloon at the room temperature. The resulting solution was stirred at room temperature for $24 \mathrm{~h}$. The reaction mixture was then purged with $\mathrm{N}_{2}$ for $30 \mathrm{~min}$ to remove most of the residual $\mathrm{SO}_{2} \mathrm{~F}_{2}$, and water was added. The mixture was acidified with concentrated $\mathrm{HCl}$, and the product was extracted into dichloromethane. The organic layer was collected, dried over $\mathrm{Na}_{2} \mathrm{SO}_{4}$, filtered and concentrated under reduced pressure. The products were purified by column chromatography on silica gel using hexanes/ethyl acetate as the eluent.

\section{Characterization of Aryl Fluorosulfates}<smiles>CC(C)(C)c1ccc(OS(=O)(=O)F)cc1</smiles>

1a was obtained as a colorless oil starting from 4-(tert-butyl)phenol (3.0 g, $20 \mathrm{mmol})$ according to the General Procedure I in 93\% yield (4.31 g). ${ }^{1} \mathrm{H}$ NMR (400 MHz, $\left.\mathrm{CDCl}_{3}\right) \delta 7.48(\mathrm{~d}, J=9.0 \mathrm{~Hz}, 2 \mathrm{H}), 7.29-7.22(\mathrm{~m}, 2 \mathrm{H}), 1.34(\mathrm{~s}, 9 \mathrm{H}) .{ }^{19} \mathrm{~F}$ NMR $(376$ $\left.\mathrm{MHz}, \mathrm{CDCl}_{3}\right) \delta 37.12 .{ }^{13} \mathrm{C} \mathrm{NMR}\left(101 \mathrm{MHz}, \mathrm{CDCl}_{3}\right) \delta 151.92,147.85,127.30,120.24$, 34.75, 31.26. HRMS (ESI-TOF) $\mathrm{m} / \mathrm{z}$ Calcd for $\mathrm{C}_{10} \mathrm{H}_{14} \mathrm{FO}_{3} \mathrm{~S}[\mathrm{M}+\mathrm{H}]^{+} 233.0642$, found 233.0644 .<smiles>COc1ccc(OS(=O)(=O)F)cc1</smiles>

1b was obtained as a colorless oil starting from 4-methoxyphenol (2.48 g, $20 \mathrm{mmol})$ according to the General Procedure I in 93\% yield (3.85 g). ${ }^{1} \mathrm{H}$ NMR (400 MHz, $\left.\mathrm{CDCl}_{3}\right) \delta 7.24(\mathrm{~d}, J=8.7 \mathrm{~Hz}, 2 \mathrm{H}), 6.92(\mathrm{~d}, J=9.0 \mathrm{~Hz}, 2 \mathrm{H}), 3.81(\mathrm{~s}, 3 \mathrm{H})$. Spectral data matched those previously reported. ${ }^{1}$<smiles>COc1cccc(OS(=O)(=O)F)c1</smiles>

1c was obtained as a colorless oil starting from methyl 3-methoxyphenol (2.48 g, 20 mmol) according to the General Procedure I in $89 \%$ yield $(4.18 \mathrm{~g}) .{ }^{1} \mathrm{H}$ NMR (400 $\left.\mathrm{MHz}, \mathrm{CDCl}_{3}\right) \delta 7.37(\mathrm{t}, J=8.3 \mathrm{~Hz}, 1 \mathrm{H}), 6.98-6.91(\mathrm{~m}, 2 \mathrm{H}), 6.87(\mathrm{~d}, J=2.1 \mathrm{~Hz}, 1 \mathrm{H})$, $3.83(\mathrm{~s}, 3 \mathrm{H})$. Spectral data matched those previously reported. ${ }^{1}$<smiles>CC(=O)Nc1ccc(OS(=O)(=O)F)cc1</smiles>

1d was obtained as a white solid starting from methyl $N$-(4-hydroxyphenyl)acetamide ( $3.02 \mathrm{~g}, 20 \mathrm{mmol}$ ) according to the General Procedure I in $76 \%$ yield $(3.55 \mathrm{~g}) .{ }^{1} \mathrm{H}$ NMR (400 MHz, $\left.\mathrm{CDCl}_{3}\right) \delta 7.62(\mathrm{~d}, J=9.0 \mathrm{~Hz}, 2 \mathrm{H}), 7.39$ (s, 1H), 7.29 (d, $J=8.8 \mathrm{~Hz}$, $2 \mathrm{H}), 2.20$ (s, 3H).Spectral data matched those previously reported. ${ }^{4}$ 
<smiles>Cc1ccc(OS(=O)(=O)F)cc1</smiles>

1e was obtained as a colorless oil starting from $p$-cresol (2.16 g, $20 \mathrm{mmol})$ according to the General Procedure I in 95\% yield (3.69 g). $\left.{ }^{1} \mathrm{H} \mathrm{NMR} \mathrm{(400} \mathrm{MHz}, \mathrm{CDCl}_{3}\right) \delta 7.24$ $(\mathrm{q}, J=8.6 \mathrm{~Hz}, 4 \mathrm{H}), 2.39(\mathrm{~s}, 3 \mathrm{H})$. Spectral data matched those previously reported. ${ }^{1}$<smiles>Cc1cc(C)cc(OS(=O)(=O)F)c1</smiles>

1f was obtained as a colorless oil starting from methyl 3,5-methylphenol (2.44 g, 20 mmol) according to the General Procedure I in $87 \%$ yield (3.56 g). ${ }^{1} \mathrm{H}$ NMR (400 $\left.\mathrm{MHz}, \mathrm{CDCl}_{3}\right) \delta 7.19(\mathrm{~d}, J=7.5 \mathrm{~Hz}, 1 \mathrm{H}), 7.11(\mathrm{~d}, J=8.9 \mathrm{~Hz}, 2 \mathrm{H}), 2.37(\mathrm{~s}, 3 \mathrm{H}), 2.34$ (s, 3H). ${ }^{19} \mathrm{~F}$ NMR $\left(376 \mathrm{MHz}, \mathrm{CDCl}_{3}\right) \delta 39.00 .{ }^{13} \mathrm{C} \mathrm{NMR}\left(101 \mathrm{MHz}, \mathrm{CDCl}_{3}\right) \delta 148.89$, 138.05, 131.84, 129.28, 127.11, 121.21, 20.81, 15.53. HRMS (ESI-TOF) m/z Calcd for $\mathrm{C}_{8} \mathrm{H}_{10} \mathrm{FO}_{3} \mathrm{~S}[\mathrm{M}+\mathrm{H}]^{+}$205.0329, found 205.0331.<smiles>O=S(=O)(F)Oc1ccccc1</smiles>

$1 \mathrm{~g}$

$1 \mathrm{~g}$ was obtained as a colorless oil starting from phenol $(1.88 \mathrm{~g}, 20 \mathrm{mmol})$ according to the General Procedure I in $75 \%$ yield $(2.69 \mathrm{~g}) .{ }^{1} \mathrm{H} \mathrm{NMR}\left(400 \mathrm{MHz}, \mathrm{CDCl}_{3}\right) \delta 7.49(\mathrm{t}$, $J=7.5 \mathrm{~Hz}, 2 \mathrm{H}), 7.42(\mathrm{t}, J=7.3 \mathrm{~Hz}, 1 \mathrm{H}), 7.35(\mathrm{~d}, J=8.2 \mathrm{~Hz}, 2 \mathrm{H})$. Spectral data matched those previously reported. ${ }^{1}$<smiles>O=S(=O)(F)Oc1ccc(-c2ccccc2)cc1</smiles>

1h was obtained as a white solid starting from [1,1'-biphenyl]-4-ol (3.4 g, $20 \mathrm{mmol})$ according to the General Procedure I in 93\% yield (4.74 g). ${ }^{1} \mathrm{H}$ NMR (400 MHz, $\left.\mathrm{CDCl}_{3}\right) \delta 7.67(\mathrm{~d}, J=8.8 \mathrm{~Hz}, 2 \mathrm{H}), 7.56(\mathrm{~d}, J=8.1 \mathrm{~Hz}, 2 \mathrm{H}), 7.48(\mathrm{t}, J=7.5 \mathrm{~Hz}, 2 \mathrm{H})$, $7.41(\mathrm{t}, J=7.6 \mathrm{~Hz}, 3 \mathrm{H})$. Spectral data matched those previously reported. ${ }^{1}$<smiles>O=S(=O)(F)Oc1cccc(F)c1</smiles>

$1 \mathbf{i}$

1i was obtained as a colorless oil starting from methyl 3-fluorophenol (2.24 g, 20 mmol) according to the General Procedure I in $84 \%$ yield $(3.27 \mathrm{~g}) .{ }^{1} \mathrm{H}$ NMR (400 $\left.\mathrm{MHz}, \mathrm{CDCl}_{3}\right) \delta 7.47(\mathrm{td}, J=8.3,6.3 \mathrm{~Hz}, 1 \mathrm{H}), 7.21-7.15(\mathrm{~m}, 2 \mathrm{H}), 7.15-7.08(\mathrm{~m}$, $1 \mathrm{H})$. Spectral data matched those previously reported. ${ }^{1}$ 
<smiles>O=S(=O)(F)Oc1cc(F)cc(F)c1</smiles>

1j was obtained as a colorless oil starting from methyl 3,5-difluorophenol (2.6 g, 20 mmol) according to the General Procedure I in $67 \%$ yield $(2.85 \mathrm{~g}) .{ }^{1} \mathrm{H}$ NMR (400 $\left.\mathrm{MHz} \mathrm{CDCl}_{3}\right) \delta 6.94(\mathrm{dd}, J=16.9,7.2 \mathrm{~Hz}, 3 \mathrm{H}) .{ }^{19} \mathrm{~F}$ NMR $\left(376 \mathrm{MHz}, \mathrm{CDCl}_{3}\right) \delta 38.52$, -105.10, -105.12, -105.12, -105.14. ${ }^{13} \mathrm{C}$ NMR $\left(101 \mathrm{MHz}, \mathrm{CDCl}_{3}\right) \delta 164.38,164.24$, $161.86,161.72,150.22,150.08,149.94,105.83,105.75,105.63,105.54,105.06$, 104.81, 104.56. HRMS (ESI-TOF) $\mathrm{m} / \mathrm{z}$ Calcd for $\mathrm{C}_{6} \mathrm{H}_{4} \mathrm{~F}_{3} \mathrm{O}_{3} \mathrm{~S}[\mathrm{M}+\mathrm{H}]^{+} 212.9828$, found 212.983 .<smiles>O=S(=O)(F)Oc1ccc(Cl)cc1</smiles>

1k was obtained as a colorless oil starting from 4-chlorophenol (2.56 g, $20 \mathrm{mmol})$ according to the General Procedure I in $86 \%$ yield $(3.61 \mathrm{~g}) .{ }^{1} \mathrm{H}$ NMR (400 MHz, $\left.\mathrm{CDCl}_{3}\right) \delta 7.41(\mathrm{dd}, J=15.4,9.0 \mathrm{~Hz}, 2 \mathrm{H}), 7.28(\mathrm{t}, J=9.2 \mathrm{~Hz}, 2 \mathrm{H})$. Spectral data matched those previously reported. ${ }^{1}$<smiles>CC(=O)c1ccc(OS(=O)(=O)F)cc1</smiles>

11 was obtained as a white solid starting from 1-(4-hydroxyphenyl)ethanone (2.72 g, $20 \mathrm{mmol}$ ) according to the General Procedure I in $85 \%$ yield (3.75 g). ${ }^{1} \mathrm{H}$ NMR (400 $\left.\mathrm{MHz} \mathrm{CDCl}_{3}\right) \delta 7.41(\mathrm{dd}, J=15.4,9.0 \mathrm{~Hz}, 2 \mathrm{H}), 7.28(\mathrm{t}, J=9.2 \mathrm{~Hz}, 2 \mathrm{H})$. Spectral data matched those previously reported. ${ }^{2}$<smiles>CC(=O)c1ccc(OS(=O)(=O)F)cc1</smiles>

1m was obtained as a colorless oil starting from methyl 4-hydroxybenzoate $(3.04 \mathrm{~g}$, $20 \mathrm{mmol}$ ) according to the General Procedure I in $89 \%$ yield $(4.18 \mathrm{~g}) .{ }^{1} \mathrm{H}$ NMR (400 $\left.\mathrm{MHz}, \mathrm{CDCl}_{3}\right) \delta 8.15(\mathrm{~d}, J=8.8 \mathrm{~Hz}, 2 \mathrm{H}), 7.41(\mathrm{~d}, J=8.5 \mathrm{~Hz}, 2 \mathrm{H}), 3.93(\mathrm{~s}, 3 \mathrm{H})$. Spectral data matched those previously reported. ${ }^{1}$<smiles>O=S(=O)(F)Oc1ccc(C(F)(F)F)cc1</smiles>

1n was obtained as a colorless oil starting from 4-hydroxybenzonitrile (3.24 g, 20 mmol) according to the General Procedure I in $83 \%$ yield (4.03 g). ${ }^{1} \mathrm{H}$ NMR (400 $\left.\mathrm{MHz}, \mathrm{CDCl}_{3}\right) \delta 7.82(\mathrm{~d}, J=8.2 \mathrm{~Hz}, 2 \mathrm{H}), 7.50(\mathrm{~d}, J=8.2 \mathrm{~Hz}, 2 \mathrm{H})$. Spectral data matched those previously reported. ${ }^{1}$ 
<smiles>N#Cc1ccc(OS(=O)(=O)F)cc1</smiles>

10 was obtained as a white solid starting from 4-hydroxybenzonitrile ( $2.38 \mathrm{~g}, 20$ mmol) according to the General Procedure I in $91 \%$ yield $(3.67 \mathrm{~g}) .{ }^{1} \mathrm{H}$ NMR (400 $\left.\mathrm{MHz}, \mathrm{CDCl}_{3}\right) \delta 7.82(\mathrm{~d}, J=8.2 \mathrm{~Hz}, 2 \mathrm{H}), 7.50(\mathrm{~d}, J=8.2 \mathrm{~Hz}, 2 \mathrm{H})$. Spectral data matched those previously reported. ${ }^{1}$<smiles>N#Cc1cccc(OS(=O)(=O)F)c1</smiles>

1p was obtained as a colorless oil starting from methyl 3-hydroxybenzonitrile (2.38 g, $20 \mathrm{mmol}$ ) according to the General Procedure I in 92\% yield (3.73 g). ${ }^{1} \mathrm{H}$ NMR (400 $\left.\mathrm{MHz}, \mathrm{CDCl}_{3}\right) \delta 7.75(\mathrm{~d}, J=6.9 \mathrm{~Hz}, 1 \mathrm{H}), 7.65(\mathrm{dd}, J=14.2,6.7 \mathrm{~Hz}, 3 \mathrm{H})$. Spectral data matched those previously reported. ${ }^{1}$<smiles>CS(=O)(=O)c1ccc(OS(=O)(=O)F)cc1</smiles>

1q was obtained as a white solid starting from methyl 4-(methylsulfonyl)phenol (3.44 g, $20 \mathrm{mmol}$ ) according to the General Procedure I in $85 \%$ yield (4.31 g). ${ }^{1} \mathrm{H}$ NMR $\left(400 \mathrm{MHz}, \mathrm{CDCl}_{3}\right) \delta 8.10(\mathrm{~d}, J=8.7 \mathrm{~Hz}, 2 \mathrm{H}), 7.57(\mathrm{~d}, J=8.6 \mathrm{~Hz}, 2 \mathrm{H}), 3.10(\mathrm{~s}, 3 \mathrm{H})$. Spectral data matched those previously reported. ${ }^{3}$<smiles>O=S(=O)(F)Oc1ccc2ccccc2c1</smiles>

1r was obtained as a colorless oil starting from naphthalen-2-ol (2.88 g, $20 \mathrm{mmol})$ according to the General Procedure I in $81 \%$ yield $(3.66 \mathrm{~g}) .{ }^{1} \mathrm{H}$ NMR (400 MHz, $\left.\mathrm{CDCl}_{3}\right) \delta 7.94(\mathrm{~d}, J=9.0 \mathrm{~Hz}, 1 \mathrm{H}), 7.92-7.85(\mathrm{~m}, 2 \mathrm{H}), 7.82(\mathrm{~s}, 1 \mathrm{H}), 7.63-7.55(\mathrm{~m}$, $2 \mathrm{H}), 7.44(\mathrm{dd}, J=9.0,2.2 \mathrm{~Hz}, 1 \mathrm{H})$. Spectral data matched those previously reported. ${ }^{1}$<smiles>O=C1CCCc2cc(OS(=O)(=O)F)ccc21</smiles>

1s was obtained as a colorless oil starting from methyl 6-hydroxy-3,4-dihydronaphthalen-1(2H)-one (3.24 g, $20 \mathrm{mmol})$ according to the General Procedure I in $87 \%$ yield $(3.56 \mathrm{~g}) .{ }^{1} \mathrm{H}$ NMR $\left(400 \mathrm{MHz}, \mathrm{CDCl}_{3}\right) \delta 8.07(\mathrm{~d}, J=$ $7.7 \mathrm{~Hz}, 1 \mathrm{H}), 7.52(\mathrm{~d}, J=8.0 \mathrm{~Hz}, 1 \mathrm{H}), 7.41(\mathrm{t}, J=7.9 \mathrm{~Hz}, 1 \mathrm{H}), 3.02(\mathrm{t}, J=5.6 \mathrm{~Hz}, 2 \mathrm{H})$, $2.73-2.61(\mathrm{~m}, 2 \mathrm{H}), 2.24-2.10(\mathrm{~m}, 2 \mathrm{H}) .{ }^{19} \mathrm{~F}$ NMR $\left(376 \mathrm{MHz}, \mathrm{CDCl}_{3}\right) \delta 39.42 .{ }^{13} \mathrm{C}$ NMR $\left(101 \mathrm{MHz}, \mathrm{cdcl}_{3}\right) \delta 196.18,147.73,136.65,134.90,127.77,127.56,125.70$, 
38.36, 23.19, 22.04. HRMS (ESI-TOF) m/z Calcd for $\mathrm{C}_{10} \mathrm{H}_{10} \mathrm{FO}_{4} \mathrm{~S}[\mathrm{M}+\mathrm{H}]^{+}$245.0278, found 245.028 .<smiles>O=c1c2ccccc2oc2cc(OS(=O)(=O)F)ccc12</smiles>

1t was obtained as a white solid starting from methyl 3-hydroxy-9H-xanthen-9-one (4.24 g, $20 \mathrm{mmol}$ ) according to the General Procedure I in $50 \%$ yield $(2.93 \mathrm{~g}) .{ }^{1} \mathrm{H}$ NMR $\left(400 \mathrm{MHz}, \mathrm{CDCl}_{3}\right) \delta 8.47(\mathrm{~d}, J=8.8 \mathrm{~Hz}, 1 \mathrm{H}), 8.34(\mathrm{~d}, J=7.8 \mathrm{~Hz}, 1 \mathrm{H}), 7.78(\mathrm{t}$, $J=7.3 \mathrm{~Hz}, 1 \mathrm{H}), 7.52(\mathrm{~d}, J=8.4 \mathrm{~Hz}, 2 \mathrm{H}), 7.44(\mathrm{t}, J=7.5 \mathrm{~Hz}, 1 \mathrm{H}), 7.36(\mathrm{~d}, J=7.8 \mathrm{~Hz}$, 1H). ${ }^{19} \mathrm{~F}$ NMR $\left(376 \mathrm{MHz}, \mathrm{CDCl}_{3}\right) \delta 39.38 .{ }^{13} \mathrm{C} \mathrm{NMR}\left(101 \mathrm{MHz}, \mathrm{CDCl}_{3}\right) \delta 175.80$, 156.56, 156.18, 153.26, 135.51, 129.70, 126.84, 124.79, 121.73, 121.62, 117.98, 116.70, 110.90. HRMS (ESI-TOF) $\mathrm{m} / \mathrm{z}$ Calcd for $\mathrm{C}_{13} \mathrm{H}_{7} \mathrm{FO}_{5} \mathrm{~S}[\mathrm{M}+\mathrm{H}]^{+}$295.0071, found 295.0073 .<smiles>CCOC(=O)c1c(C)n(C)c2ccc(OS(=O)(=O)F)cc12</smiles>

1u was obtained as a colorless oil starting from methyl 3-hydroxy-9H-xanthen-9-one (4.66 g, $20 \mathrm{mmol}$ ) according to the General Procedure I in $57 \%$ yield $(3.59 \mathrm{~g}) .{ }^{1} \mathrm{H}$ NMR (400 MHz, $\left.\mathrm{CDCl}_{3}\right) \delta 8.08(\mathrm{~s}, 1 \mathrm{H}), 7.30(\mathrm{~d}, J=8.8 \mathrm{~Hz}, 1 \mathrm{H}), 7.17(\mathrm{~d}, J=8.1 \mathrm{~Hz}$, $1 \mathrm{H}), 4.40(\mathrm{dd}, J=14.1,7.0 \mathrm{~Hz}, 2 \mathrm{H}), 3.70(\mathrm{~s}, 3 \mathrm{H}), 2.77(\mathrm{~s}, 3 \mathrm{H}), 1.45$ (t, $J=7.0 \mathrm{~Hz}$, 3H). ${ }^{19} \mathrm{~F} \mathrm{NMR}\left(376 \mathrm{MHz}, \mathrm{CDCl}_{3}\right) \delta 36.56 .{ }^{13} \mathrm{C} \mathrm{NMR}\left(101 \mathrm{MHz}, \mathrm{CDCl}_{3}\right) \delta 165.26$, 147.52, 145.61, 135.33, 127.06, 114.53, 113.63, 110.14, 104.73, 59.77, 29.93, 14.49, 12.01. HRMS (ESI-TOF) $\mathrm{m} / \mathrm{z}$ Calcd for $\mathrm{C}_{13} \mathrm{H}_{18} \mathrm{FN}_{2} \mathrm{O}_{5} \mathrm{~S}\left[\mathrm{M}+\mathrm{NH}_{4}\right]^{+} 333.0915$, found 333.091 .<smiles>Cc1ccccc1OS(=O)(=O)F</smiles>

1v was obtained as a colorless oil starting from $o$-cresol $(2.16 \mathrm{~g}, 20 \mathrm{mmol})$ according to the General Procedure I in 52\% yield (1.97 g). ${ }^{1} \mathrm{H}$ NMR $\left(400 \mathrm{MHz}, \mathrm{CDCl}_{3}\right) \delta 7.31$ $-7.26(\mathrm{~m}, 4 \mathrm{H}), 2.36(\mathrm{~s}, 3 \mathrm{H})$. Spectral data matched those previously reported. ${ }^{1}$<smiles>O=S(=O)(F)Oc1ccccc1F</smiles>

1w was obtained as a colorless oil starting from 2-fluorophenol (2.24 g, $20 \mathrm{mmol})$ according to the General Procedure I in $71 \%$ yield $(2.77 \mathrm{~g}) .{ }^{1} \mathrm{H}$ NMR (400 MHz, 
$\left.\mathrm{CDCl}_{3}\right) \delta 7.47-7.35(\mathrm{~m}, 2 \mathrm{H}), 7.33-7.20(\mathrm{~m}, 2 \mathrm{H}) .{ }^{1} \mathrm{H}$ NMR $\left(400 \mathrm{MHz}, \mathrm{CDCl}_{3}\right) \delta$ $7.46-7.36(\mathrm{~m}, 2 \mathrm{H}), 7.31-7.22(\mathrm{~m}, 2 \mathrm{H}) .{ }^{19} \mathrm{~F} \mathrm{NMR}\left(376 \mathrm{MHz}, \mathrm{CDCl}_{3}\right) \delta 39.03(\mathrm{~d}, J=$ $10.3 \mathrm{~Hz}),-127.81--128.09(\mathrm{~m}) .{ }^{13} \mathrm{C} \mathrm{NMR}\left(101 \mathrm{MHz}, \mathrm{CDCl}_{3}\right) \delta 154.83,152.30$, 130.12, 130.05, 125.16, 125.11, 123.23, 117.99, 117.82. HRMS (ESI-TOF) m/z Calcd for $\mathrm{C}_{6} \mathrm{H}_{5} \mathrm{~F}_{2} \mathrm{O}_{3} \mathrm{~S}[\mathrm{M}+\mathrm{H}]^{+}$194.9922, found 194.9923 .<smiles>O=S(=O)(F)Oc1ccccc1-c1ccccc1</smiles>

1x was obtained as a white solid starting from [1,1'-biphenyl]-2-ol (2.16 g, $20 \mathrm{mmol})$ according to the General Procedure I in $62 \%$ yield $(3.12 \mathrm{~g}) .{ }^{1} \mathrm{H}$ NMR (400 MHz, $\left.\mathrm{CDCl}_{3}\right) \delta 7.51-7.40(\mathrm{~m}, 9 \mathrm{H})$. Spectral data matched those previously reported. ${ }^{1}$<smiles>CCCCC(=O)OC1CCC2C3CCc4cc(OS(=O)(=O)F)ccc4C3CCC12C</smiles>

$1 y$

$1 \mathbf{y}$ was obtained as a colorless oil starting from oestradiol 17-heptanoate (3.84 g, 10 mmol) according to the General Procedure I in $86 \%$ yield $(4.0 \mathrm{~g}) .{ }^{1} \mathrm{H} \mathrm{NMR}(400 \mathrm{MHz}$, $\left.\mathrm{CDCl}_{3}\right) \delta 7.35(\mathrm{~d}, J=8.7 \mathrm{~Hz}, 1 \mathrm{H}), 7.08(\mathrm{~d}, J=8.6 \mathrm{~Hz}, 1 \mathrm{H}), 7.03(\mathrm{~s}, 1 \mathrm{H}), 4.71(\mathrm{t}, J=$ $8.5 \mathrm{~Hz}, 1 \mathrm{H}), 2.90(\mathrm{dd}, J=8.5,4.0 \mathrm{~Hz}, 2 \mathrm{H}), 2.34-2.16(\mathrm{~m}, 5 \mathrm{H}), 1.96-1.86(\mathrm{~m}, 2 \mathrm{H})$, $1.81-1.70(\mathrm{~m}, 1 \mathrm{H}), 1.63(\mathrm{dt}, J=11.2,5.6 \mathrm{~Hz}, 2 \mathrm{H}), 1.59-1.22(\mathrm{~m}, 13 \mathrm{H}), 0.89(\mathrm{t}, J=$ $6.7 \mathrm{~Hz}, 3 \mathrm{H}), 0.83(\mathrm{~s}, 3 \mathrm{H}) .{ }^{19} \mathrm{~F} \mathrm{NMR}\left(376 \mathrm{MHz}, \mathrm{CDCl}_{3}\right) \delta 37.28 .{ }^{13} \mathrm{C} \mathrm{NMR}(101 \mathrm{MHz}$, $\left.\mathrm{cdcl}_{3}\right) \delta 173.93,147.98,141.02,139.58,127.33,120.61,117.63,82.18,49.77,43.93$, $42.85,37.94,36.78,34.58,31.47,29.49,28.82,27.54,26.74,25.98,25.08,23.24$, $22.52,14.05,12.04$. HRMS (ESI-TOF) $\mathrm{m} / \mathrm{z}$ Calcd for $\mathrm{C}_{25} \mathrm{H}_{39} \mathrm{FNO}_{5} \mathrm{~S}\left[\mathrm{M}+\mathrm{NH}_{4}\right]^{+}$ 484.2527, found 484.2527 .

\section{The Synthesis of Pyridinyl Fluorosulfates}

\subsection{Scope of Pyridinyl Fluorosulfates}


<smiles>COc1ccnc(OC)c1</smiles>

$4 a$<smiles>Cc1ncccc1OC(F)(F)F</smiles>

$4 e$<smiles>[O-]c1cccnc1</smiles>

$4 i$<smiles>COc1ccc(OC(F)(F)F)cn1</smiles>

4b<smiles>COc1ccc(C)nc1C</smiles>

$4 f$<smiles>COc1cc(C)nc(C)c1</smiles>

4j<smiles>COc1cncc(OC(F)(F)F)c1</smiles>

4c<smiles>CCc1nc(C)ccc1OC(F)(F)F</smiles>

$4 g$<smiles>Cc1cccc(O[Se-])n1</smiles>

4k<smiles>Cc1ccc(OC(F)(F)F)cn1</smiles>

4d

4h<smiles>[O-]c1ccccn1</smiles>

41

\subsection{General Procedure II for the Synthesis of Pyridinyl Fluorosulfates}

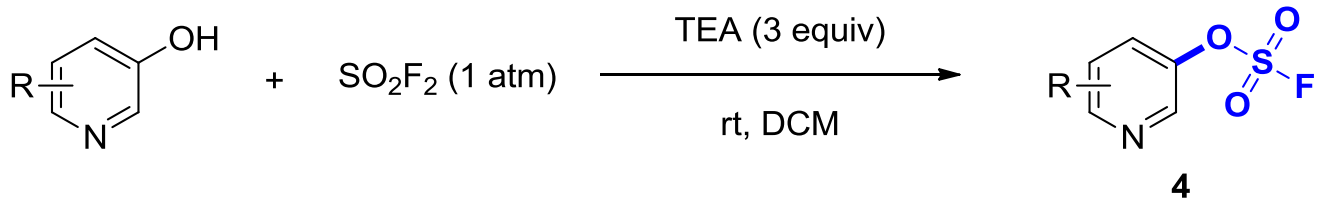

All the aryl fluorosulfates were synthesized according to literature ${ }^{2}$. A three-neck round-bottom flask equipped with a thermometer was charged with the corresponding pyridinol, TEA (3.03 g, 1.5 equiv, $30 \mathrm{mmol})$, DCM $(50 \mathrm{~mL})$. The flask was capped with a rubber septum. Then $\mathrm{SO}_{2} \mathrm{~F}_{2}$ gas was introduced into the stirring reaction mixture by slow bubbling through a $\mathrm{SO}_{2} \mathrm{~F}_{2}$ balloon at the room temperature. The reaction mixture was vigorously stirred at room temperature for $24 \mathrm{~h}$, monitoring by TLC. After completion, the solvent was removed by rotary evaporation. The residue was dissolved in EtOAc, washed with saturated sodium bicarbonate and brine, dried over $\mathrm{Na}_{2} \mathrm{SO}_{4}$, filtered and concentrated under reduced pressure. The residue was purified by column chromatography on silica gel using hexanes/ethyl acetate as the eluent.

\subsection{Synthesis of $4 h$}
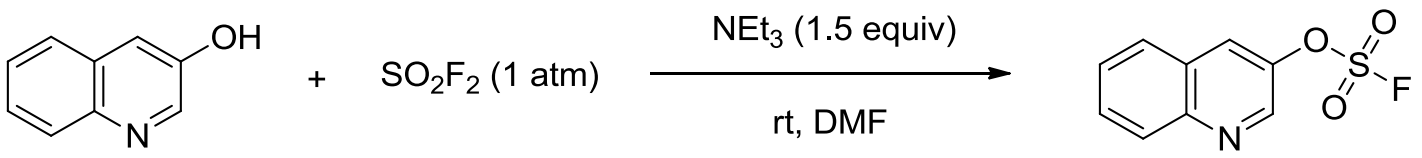

4h

A three-neck round-bottom flask equipped with a thermometer was charged with the quinolin-3-ol (2.9 g, $20 \mathrm{mmol})$, TEA (3.03 g, 1.5 equiv, $30 \mathrm{mmol})$, DMF (20 mL). The flask was capped with a rubber septum. Then $\mathrm{SO}_{2} \mathrm{~F}_{2}$ gas was introduced into the stirring reaction mixture by slow bubbling through a $\mathrm{SO}_{2} \mathrm{~F}_{2}$ balloon at the room 
temperature. The reaction mixture was vigorously stirred at room temperature for 24 hours, monitoring by TLC. After completion, the mixture was extracted with DCM (50 $\mathrm{mL} \times 3)$, and washed with saturated sodium bicarbonate and brine, dried over $\mathrm{Na}_{2} \mathrm{SO}_{4}$, filtered and concentrated under reduced pressure. The residue was purified by column chromatography on silica gel using hexanes/ethyl acetate as the eluent to give the product as a white solid in $62 \%$ yield $(2.77 \mathrm{~g})$.

\section{Characterization of Pyridinyl Fluorosulfates}<smiles>COc1cc(OS(=O)(=O)F)ccn1</smiles>

4a was obtained as a colorless oil starting from 6-methoxypyridin-3-ol (2.5 g, 20 mmol) according to the General Procedure II in 57\% yield $(2.35 \mathrm{~g}) .{ }^{1} \mathrm{H}$ NMR (400 $\left.\mathrm{MHz}, \mathrm{CDCl}_{3}\right) \delta 8.26(\mathrm{~d}, J=5.7 \mathrm{~Hz}, 1 \mathrm{H}), 6.88(\mathrm{~d}, J=5.5 \mathrm{~Hz}, 1 \mathrm{H}), 6.71(\mathrm{~s}, 1 \mathrm{H}), 3.97$ (s, 3H). ${ }^{19} \mathrm{~F}$ NMR $\left(376 \mathrm{MHz}, \mathrm{CDCl}_{3}\right) \delta 40.12 .{ }^{13} \mathrm{C} \mathrm{NMR}\left(101 \mathrm{MHz}, \mathrm{CDCl}_{3}\right) \delta 165.91$, 157.79, 149.43, 108.92, 102.83, 77.34, 77.02, 76.70, 54.34. HRMS (ESI-TOF) m/z Calcd for $\mathrm{C}_{6} \mathrm{H}_{7} \mathrm{FNO}_{4} \mathrm{~S}[\mathrm{M}+\mathrm{H}]^{+} 208.0074$, found 208.0077.<smiles>COc1ccc(OS(=O)(=O)F)cn1</smiles>

4b was obtained as a white solid starting from 6-methoxypyridin-3-ol (2.5 g, 20 mmol) according to the General Procedure II in $73 \%$ yield $(3.02 \mathrm{~g}) .{ }^{1} \mathrm{H}$ NMR (400 MHz, $\left.\mathrm{CDCl}_{3}\right) \delta 8.20(\mathrm{~s}, 1 \mathrm{H}), 7.56(\mathrm{~d}, J=8.9 \mathrm{~Hz}, 1 \mathrm{H}), 6.82(\mathrm{~d}, J=9.0 \mathrm{~Hz}, 1 \mathrm{H}), 3.95(\mathrm{~s}, 3 \mathrm{H})$. ${ }^{19} \mathrm{~F}$ NMR $\left(376 \mathrm{MHz}, \mathrm{CDCl}_{3}\right) \delta 36.75 .{ }^{13} \mathrm{C} \mathrm{NMR}\left(101 \mathrm{MHz}, \mathrm{CDCl}_{3}\right) \delta 163.32,141.94$, 139.35, 131.59, 112.32, 54.20. HRMS (ESI-TOF) m/z Calcd for $\mathrm{C}_{6} \mathrm{H}_{7} \mathrm{FNO}_{4} \mathrm{~S}[\mathrm{M}+\mathrm{H}]^{+}$ 208.0074, found 208.0077 .<smiles>COc1cncc(OS(=O)(=O)F)c1</smiles>

4c was obtained as a white solid starting from 5-methoxypyridin-3-ol (2.5 g, 20 mmol) according to the General Procedure II in $78 \%$ yield $(3.23 \mathrm{~g}) .{ }^{1} \mathrm{H}$ NMR (400 MHz, $\left.\mathrm{CDCl}_{3}\right) \delta 8.39(\mathrm{~s}, 1 \mathrm{H}), 8.29(\mathrm{~s}, 1 \mathrm{H}), 7.19(\mathrm{~s}, 1 \mathrm{H}), 3.91(\mathrm{~s}, 3 \mathrm{H}) .{ }^{19} \mathrm{~F}$ NMR $(376 \mathrm{MHz}$, $\left.\mathrm{CDCl}_{3}\right) \delta 38.45 .{ }^{13} \mathrm{C} \mathrm{NMR}\left(101 \mathrm{MHz}, \mathrm{CDCl}_{3}\right) \delta 156.45,147.09,138.15,133.98$, 113.33, 56.20. HRMS (ESI-TOF) $\mathrm{m} / \mathrm{z}$ Calcd for $\mathrm{C}_{6} \mathrm{H}_{7} \mathrm{FNO}_{4} \mathrm{~S}[\mathrm{M}+\mathrm{H}]^{+}$208.0074, found 208.0072 . 
<smiles>Cc1ccc(OS(=O)(=O)F)cn1</smiles>

4d was obtained as a colorless oil starting from 6-methylpyridin-3-ol (2.18 g, 20 mmol) according to the General Procedure II in $85 \%$ yield (3.24 g). ${ }^{1} \mathrm{H}$ NMR (400 $\left.\mathrm{MHz} \mathrm{CDCl}_{3}\right) \delta 8.54(\mathrm{~s}, 1 \mathrm{H}), 7.59(\mathrm{dd}, J=8.3,1.8 \mathrm{~Hz}, 1 \mathrm{H}), 7.29(\mathrm{~d}, J=8.7 \mathrm{~Hz}, 1 \mathrm{H})$, $2.62(\mathrm{~s}, 3 \mathrm{H})$. Spectral data matched those previously reported. ${ }^{5}$<smiles>Cc1ncccc1OS(=O)(=O)F</smiles>

4e was obtained as a colorless oil starting from 2-methylpyridin-3-ol (2.18 g, 20 mmol) according to the General Procedure II in $76 \%$ yield $(3.14 \mathrm{~g}) .{ }^{1} \mathrm{H}$ NMR (400 $\left.\mathrm{MHz}, \mathrm{CDCl}_{3}\right) \delta 8.56(\mathrm{~d}, J=4.4 \mathrm{~Hz}, 1 \mathrm{H}), 7.67(\mathrm{~d}, J=8.3 \mathrm{~Hz}, 1 \mathrm{H}), 7.29(\mathrm{dd}, J=8.1$, $4.9 \mathrm{~Hz}, 1 \mathrm{H}), 2.65(\mathrm{~s}, 3 \mathrm{H}) .{ }^{19} \mathrm{~F}$ NMR $\left(376 \mathrm{MHz}, \mathrm{CDCl}_{3}\right) \delta 39.86 .{ }^{13} \mathrm{C} \mathrm{NMR}(101 \mathrm{MHz}$, $\left.\mathrm{CDCl}_{3}\right) \delta 151.59,149.12,145.78,128.75,122.64,19.37$. HRMS (ESI-TOF) $\mathrm{m} / \mathrm{z}$ Calcd for $\mathrm{C}_{6} \mathrm{H}_{7} \mathrm{FNO}_{3} \mathrm{~S}[\mathrm{M}+\mathrm{H}]^{+}$192.0125, found 192.0123.<smiles>Cc1ccc(OS(=O)(=O)F)c(C)n1</smiles>

4f was obtained as a colorless oil starting from 2,6-dimethylpyridin-3-ol (2.46 g, 20 mmol) according to the General Procedure II in $67 \%$ yield $(2.74 \mathrm{~g}) .{ }^{1} \mathrm{H}$ NMR (400 $\left.\mathrm{MHz}, \mathrm{CDCl}_{3}\right) \delta 7.49(\mathrm{~d}, J=8.1 \mathrm{~Hz}, 1 \mathrm{H}), 7.07(\mathrm{~d}, J=8.1 \mathrm{~Hz}, 1 \mathrm{H}), 2.55(\mathrm{~d}, J=6.1 \mathrm{~Hz}$, $6 \mathrm{H}) .{ }^{19} \mathrm{~F} \mathrm{NMR}\left(376 \mathrm{MHz}, \mathrm{CDCl}_{3}\right) \delta 39.29 .{ }^{13} \mathrm{C} \mathrm{NMR}\left(101 \mathrm{MHz}, \mathrm{CDCl}_{3}\right) \delta 158.43$, 150.34, 143.91, 128.85, 122.14, 23.98, 19.22. HRMS (ESI-TOF) $\mathrm{m} / \mathrm{z}$ Calcd for $\mathrm{C}_{7} \mathrm{H}_{9} \mathrm{FNO}_{3} \mathrm{~S}[\mathrm{M}+\mathrm{H}]^{+}$206.0282, found 206.0279.<smiles>CCc1nc(C)ccc1OS(=O)(=O)F</smiles>

4g was obtained as a colorless oil starting from 2-ethyl-6-methylpyridin-3-ol (2.74 g, $20 \mathrm{mmol}$ ) according to the General Procedure II in $71 \%$ yield (3.10 g). ${ }^{1} \mathrm{H}$ NMR (400 $\left.\mathrm{MHz}, \mathrm{CDCl}_{3}\right) \delta 7.49(\mathrm{~d}, J=8.4 \mathrm{~Hz}, 1 \mathrm{H}), 7.06(\mathrm{~d}, J=8.4 \mathrm{~Hz}, 1 \mathrm{H}), 2.85(\mathrm{q}, J=7.6 \mathrm{~Hz}$, $2 \mathrm{H}), 2.54(\mathrm{~s}, 3 \mathrm{H}), 1.28(\mathrm{t}, J=7.6 \mathrm{~Hz}, 3 \mathrm{H}) .{ }^{19} \mathrm{~F} \mathrm{NMR}\left(376 \mathrm{MHz}, \mathrm{CDCl}_{3}\right) \delta 39.44 .{ }^{13} \mathrm{C}$ NMR $\left(101 \mathrm{MHz}, \mathrm{CDCl}_{3}\right) \delta 158.54,154.81,143.48,128.67,122.02,25.57,24.05$. HRMS (ESI-TOF) m/z Calcd for $\mathrm{C}_{8} \mathrm{H}_{11} \mathrm{FNO}_{3} \mathrm{~S}[\mathrm{M}+\mathrm{H}]^{+} 220.0438$, found 220.0436. 


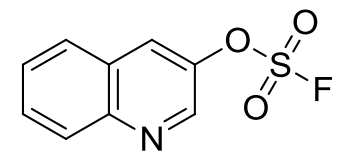

$4 \mathrm{~h}$

4h was obtained as a white solid. ${ }^{1} \mathrm{H}$ NMR $\left(400 \mathrm{MHz}, \mathrm{CDCl}_{3}\right) \delta 8.91(\mathrm{~s}, 1 \mathrm{H}), 8.18$ $(\mathrm{d}, J=13.8 \mathrm{~Hz}, 2 \mathrm{H}), 7.94-7.77(\mathrm{~m}, 2 \mathrm{H}), 7.67(\mathrm{t}, J=7.0 \mathrm{~Hz}, 1 \mathrm{H}) .{ }^{19} \mathrm{~F}$ NMR $(376$ $\left.\mathrm{MHz}, \mathrm{CDCl}_{3}\right) \delta 38.45 .{ }^{13} \mathrm{C} \mathrm{NMR}\left(101 \mathrm{MHz}, \mathrm{CDCl}_{3}\right) \delta 147.30,143.47,143.37,130.86$, 129.68, 128.45, 128.01, 127.51, 126.58. HRMS (ESI-TOF) $\mathrm{m} / \mathrm{z}$ Calcd for $\mathrm{C}_{9} \mathrm{H}_{7} \mathrm{FNO}_{3} \mathrm{~S}[\mathrm{M}+\mathrm{H}]^{+} 228.0125$, found 228.0123.<smiles>O=S(=O)(F)Oc1cccnc1</smiles>

4i was obtained as a colorless oil starting from pyridin-3-ol (1.9 g, $20 \mathrm{mmol})$ according to the General Procedure II in $80 \%$ yield (2.82 g). ${ }^{1} \mathrm{H}$ NMR (400 MHz, $\left.\mathrm{CDCl}_{3}\right) \delta 8.71-8.62(\mathrm{~m}, 2 \mathrm{H}), 7.74-7.65(\mathrm{~m}, 1 \mathrm{H}), 7.45(\mathrm{dd}, J=8.5,4.8 \mathrm{~Hz}, 1 \mathrm{H})$. Spectral data matched those previously reported. ${ }^{5}$<smiles>Cc1cc(OS(=O)(=O)F)cc(C)n1</smiles>

4j was obtained as a colorless oil starting from 2,6-dimethylpyridin-3-ol (2.46 g, 20 mmol) according to the General Procedure II in 53\% yield $(2.17 \mathrm{~g}) .{ }^{1} \mathrm{H}$ NMR (400 $\left.\mathrm{MHz}, \mathrm{CDCl}_{3}\right) \delta 6.93(\mathrm{~s}, 2 \mathrm{H}), 2.56(\mathrm{~s}, 6 \mathrm{H}) .{ }^{19} \mathrm{~F} \mathrm{NMR}\left(376 \mathrm{MHz}, \mathrm{CDCl}_{3}\right) \delta 40.05 .{ }^{13} \mathrm{C}$ NMR $\left(101 \mathrm{MHz}, \mathrm{CDCl}_{3}\right) \delta 161.38,157.00,111.81,24.60$. HRMS (ESI-TOF) m/z Calcd for $\mathrm{C}_{7} \mathrm{H}_{9} \mathrm{FNO}_{3} \mathrm{~S}[\mathrm{M}+\mathrm{H}]^{+}$206.0282, found 206.028.<smiles>Cc1cccc(OS(=O)(=O)F)n1</smiles>

4k was obtained as a colorless oil starting from 6-methylpyridin-2-ol (2.18 g, 20 mmol) according to the General Procedure II in $89 \%$ yield (3.39 g). ${ }^{1} \mathrm{H}$ NMR (400 $\left.\mathrm{MHz}, \mathrm{CDCl}_{3}\right) \delta 7.78(\mathrm{t}, J=7.8 \mathrm{~Hz}, 1 \mathrm{H}), 7.25(\mathrm{t}, J=6.0 \mathrm{~Hz}, 1 \mathrm{H}), 6.99(\mathrm{~d}, J=8.1 \mathrm{~Hz}$, $1 \mathrm{H}), 2.56$ (s, 3H). Spectral data matched those previously reported. ${ }^{5}$<smiles>O=S(=O)(F)Oc1ccccn1</smiles>

4l was obtained as a colorless oil starting from pyridin-2-ol (1.9 g, $20 \mathrm{mmol})$ according to the General Procedure II in $84 \%$ yield (2.97 g). ${ }^{1} \mathrm{H}$ NMR (400 MHz, 
$\left.\mathrm{CDCl}_{3}\right) \delta 8.39(\mathrm{dd}, J=4.6,1.5 \mathrm{~Hz}, 1 \mathrm{H}), 7.91(\mathrm{td}, J=8.1,1.9 \mathrm{~Hz}, 1 \mathrm{H}), 7.40(\mathrm{dd}, J=$ 7.3, $4.9 \mathrm{~Hz}, 1 \mathrm{H}), 7.18(\mathrm{~d}, J=8.2 \mathrm{~Hz}, 1 \mathrm{H})$. Spectral data matched those previously reported. $^{5}$

\section{General Procedure for the Carboxylation of Aryl Fluorosulfates}

General Procedure III: All operations were carried out under standard Schlenk and glovebox techniques. In a $\mathrm{N}_{2}$-filled glovebox, to a $50 \mathrm{~mL}$ Schlenk tube with a stir bar was added the $\mathrm{Ni}\left(\mathrm{PPh}_{3}\right)_{2} \mathrm{Cl}_{2}$ (16.3 mg, $0.025 \mathrm{mmol}, 0.05$ equiv), Neocuproine (10.4 $\mathrm{mg}, 0.05 \mathrm{mmol}, 0.1$ equiv), and $\mathrm{Mn}$ powder ( $82.3 \mathrm{mg}, 1.5 \mathrm{mmol}, 3$ equiv). Then the Schlenk tube was stirred and evacuated under the vacuum pump for 30 minutes. Then $\mathrm{CO}_{2}$ gas was introduced into the stirring reaction mixture for three times. After that DMF $(0.5 \mathrm{~mL})$ was injected into the tube under the $\mathrm{CO}_{2}$ atmosphere. Then the reaction mixture was stirred for 10 minutes and the mixture was gradually turned into dark blue. Then aryl fluorosulfates $(0.5 \mathrm{mmol})$ and another DMF $(0.5 \mathrm{~mL})$ were introduced into the Schlenk tube under the $\mathrm{CO}_{2}$ atmosphere. Then the resulting solution was stirred at room temperature for $20 \mathrm{~h}$. After completion of the reaction, the reaction mixture was quenched with $6 \mathrm{M} \mathrm{HCl}$ and extracted with EtOAc $(30 \mathrm{~mL} \mathrm{x}$ 5). The organic layer was collected, dried over $\mathrm{Na}_{2} \mathrm{SO}_{4}$, filtered and concentrated under reduced pressure. The products were purified by column chromatography on silica gel using hexanes/ethyl acetate as the eluent.

\section{Characterization Data for the Carboxylative Products of Aryl}

\section{Fluorosulfates}<smiles>CC(C)(C)c1ccc(C(=O)O)cc1</smiles>

2a

2a was obtained as a white solid starting from $1 \mathbf{a}(116 \mathrm{mg}, 0.5 \mathrm{mmol})$ according to the General Procedure III in $96 \%$ yield $(85.4 \mathrm{mg}) .{ }^{1} \mathrm{H}$ NMR $\left(400 \mathrm{MHz}, \mathrm{CDCl}_{3}\right) \delta 12.63$ (brs, 1H), $8.04(\mathrm{~d}, J=7.8 \mathrm{~Hz}, 2 \mathrm{H}), 7.48(\mathrm{~d}, J=7.9 \mathrm{~Hz}, 2 \mathrm{H}), 1.34(\mathrm{~s}, 9 \mathrm{H}) .{ }^{13} \mathrm{C} \mathrm{NMR}$ $\left(101 \mathrm{MHz}, \mathrm{CDCl}_{3}\right) \delta 172.43,157.57,130.10,126.50,125.46,35.18,31.08$. Spectral data matched those previously reported. ${ }^{6}$<smiles>COc1ccc(C(=O)O)cc1</smiles>

2b

2b was obtained as a white solid starting from $\mathbf{1 b}(103 \mathrm{mg}, 0.5 \mathrm{mmol})$ according to the General Procedure III in 94\% yield $(71.3 \mathrm{mg}) .{ }^{1} \mathrm{H}$ NMR $\left(400 \mathrm{MHz}, \mathrm{DMSO}-\mathrm{d}_{6}\right) \delta$ $12.64(\mathrm{~s}, 1 \mathrm{H}), 7.90(\mathrm{~d}, J=8.7 \mathrm{~Hz}, 2 \mathrm{H}), 7.01(\mathrm{~d}, J=8.7 \mathrm{~Hz}, 2 \mathrm{H}), 3.82(\mathrm{~s}, 3 \mathrm{H}) .{ }^{13} \mathrm{C}$ NMR (101 MHz, DMSO-d $\left.{ }_{6}\right) \delta$ 167.03, 162.84, 131.37, 122.97, 113.81, 55.44. Spectral data matched those previously reported. ${ }^{6}$ 
<smiles>COc1cccc(C(=O)O)c1</smiles>

2c

2c was obtained as a white solid starting from 1c (103 mg, $0.5 \mathrm{mmol})$ according to the General Procedure III in $89 \%$ yield $(67.5 \mathrm{mg}) .{ }^{1} \mathrm{H}$ NMR $\left(400 \mathrm{MHz}, \mathrm{DMSO}-\mathrm{d}_{6}\right) \delta 7.57$ $(\mathrm{d}, J=7.3 \mathrm{~Hz}, 1 \mathrm{H}), 7.48(\mathrm{~s}, 1 \mathrm{H}), 7.44(\mathrm{t}, J=7.9 \mathrm{~Hz}, 1 \mathrm{H}), 7.22(\mathrm{~d}, J=8.0 \mathrm{~Hz}, 1 \mathrm{H})$, $3.84(\mathrm{~s}, 3 \mathrm{H}) .{ }^{13} \mathrm{C}$ NMR (101 MHz, DMSO-d 6 ) $\delta 167.17,159.24,132.20,129.73$, $121.58,118.91,113.91,55.25$. Spectral data matched those previously reported. ${ }^{6}$<smiles>CC(=O)Nc1ccc(C(=O)O)cc1</smiles>

2d was obtained as a white solid starting from $1 \mathbf{1 d}(117 \mathrm{mg}, 0.5 \mathrm{mmol})$ according to the General Procedure III in $83 \%$ yield $(74.1 \mathrm{mg}) .{ }^{1} \mathrm{H}$ NMR $\left(400 \mathrm{MHz}, \mathrm{DMSO}-\mathrm{d}_{6}\right) \delta$ 12.75 (brs, 1H), 10.29 (s, 1H), 7.92 (d, $J=8.4 \mathrm{~Hz}, 2 \mathrm{H}), 7.73$ (d, $J=8.4 \mathrm{~Hz}, 2 \mathrm{H}), 2.11$ (s, 3H). ${ }^{13} \mathrm{C}$ NMR (101 MHz, DMSO-d 6 ) $\delta$ 168.93, 167.02, 143.40, 130.45, 124.91, $118.20,24.23$. Spectral data matched those previously reported. ${ }^{8}$

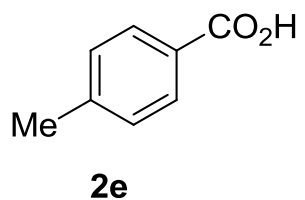

2e was obtained as a white solid starting from 1e $(95 \mathrm{mg}, 0.5 \mathrm{mmol})$ according to the General Procedure III in $96 \%$ yield $(65.2 \mathrm{mg}) .{ }^{1} \mathrm{H}$ NMR $\left(400 \mathrm{MHz}, \mathrm{CDCl}_{3}\right) \delta 8.01(\mathrm{~d}$, $J=7.8 \mathrm{~Hz}, 2 \mathrm{H}), 7.27(\mathrm{~d}, J=7.7 \mathrm{~Hz}, 2 \mathrm{H}), 2.43(\mathrm{~s}, 3 \mathrm{H}) .{ }^{13} \mathrm{C} \mathrm{NMR}\left(101 \mathrm{MHz}, \mathrm{CDCl}_{3}\right) \delta$ $172.57,144.65,130.24,129.19,126.57,21.76$. Spectral data matched those previously reported. ${ }^{6}$<smiles>CCCCCCC</smiles>

2f was obtained as a white solid starting from $\mathbf{1 f}(102 \mathrm{mg}, 0.5 \mathrm{mmol})$ according to the General Procedure III in 92\% yield $(69 \mathrm{mg}) .{ }^{1} \mathrm{H}$ NMR $\left(400 \mathrm{MHz}, \mathrm{CDCl}_{3}\right) \delta 12.72(\mathrm{~s}$, 1H), $7.88(\mathrm{~s}, 1 \mathrm{H}), 7.25(\mathrm{~d}, J=7.9 \mathrm{~Hz}, 1 \mathrm{H}), 7.15(\mathrm{~d}, J=7.5 \mathrm{~Hz}, 1 \mathrm{H}), 2.60$ (s, 3H), 2.35 $(\mathrm{s}, 3 \mathrm{H}) .{ }^{13} \mathrm{C} \mathrm{NMR}\left(101 \mathrm{MHz}, \mathrm{CDCl}_{3}\right) \delta 173.78,138.23,135.39,133.75,131.98$, $131.84,128.03,21.67,20.75$. Spectral data matched those previously reported. ${ }^{11}$<smiles>O=C(O)c1ccccc1</smiles>

$2 \mathrm{~g}$

$\mathbf{2 g}$ was obtained as a white solid starting from $1 \mathrm{~g}(88 \mathrm{mg}, 0.5 \mathrm{mmol})$ according to the General Procedure III in 95\% yield (58.9 mg). ${ }^{1} \mathrm{H}$ NMR (400 MHz, $\left.\mathrm{CDCl}_{3}\right) \delta 12.76$ 
(brs, $1 \mathrm{H}), 8.15(\mathrm{~d}, J=8.0 \mathrm{~Hz}, 2 \mathrm{H}), 7.63(\mathrm{t}, J=6.9 \mathrm{~Hz}, 1 \mathrm{H}), 7.49(\mathrm{t}, J=7.5 \mathrm{~Hz}, 2 \mathrm{H})$. ${ }^{13} \mathrm{C} \mathrm{NMR}\left(101 \mathrm{MHz}, \mathrm{CDCl}_{3}\right) \delta 172.64,133.86,130.23,129.31,128.50$. Spectral data matched those previously reported. ${ }^{6}$<smiles>O=C(O)c1ccc(-c2ccccc2)cc1</smiles>

2h

2h was obtained as a white solid starting from $\mathbf{1 h}(126 \mathrm{mg}, 0.5 \mathrm{mmol})$ according to the General Procedure III in 97\% yield $(95.7 \mathrm{mg}) .{ }^{1} \mathrm{H}$ NMR $\left(400 \mathrm{MHz}, \mathrm{DMSO}-\mathrm{d}_{6}\right) \delta$ $13.06(\mathrm{~s}, 1 \mathrm{H}), 8.06(\mathrm{~d}, J=8.1 \mathrm{~Hz}, 2 \mathrm{H}), 7.84(\mathrm{~d}, J=8.0 \mathrm{~Hz}, 2 \mathrm{H}), 7.77(\mathrm{~d}, J=7.4 \mathrm{~Hz}$, 2H), 7.54 (t, $J=7.4 \mathrm{~Hz}, 2 \mathrm{H}), 7.47$ (d, $J=7.2 \mathrm{~Hz}, 1 \mathrm{H}) .{ }^{13} \mathrm{C}$ NMR $(101 \mathrm{MHz}$, DMSO-d $\left._{6}\right) \delta 167.18,144.33,139.04,130.00,129.62,129.12,128.33,127.00,126.85$. Spectral data matched those previously reported. ${ }^{6}$<smiles>O=C(O)c1cccc(F)c1</smiles>

2i

$2 \mathbf{i}$ was obtained as a white solid starting from $1 \mathbf{i}(97 \mathrm{mg}, 0.5 \mathrm{mmol})$ according to the General Procedure III in $87 \%$ yield $(60.8 \mathrm{mg}) .{ }^{1} \mathrm{H} \mathrm{NMR}\left(400 \mathrm{MHz}, \mathrm{CDCl}_{3}\right) \delta 11.90(\mathrm{~s}$, $1 \mathrm{H}), 7.92(\mathrm{~d}, J=7.5 \mathrm{~Hz}, 1 \mathrm{H}), 7.80(\mathrm{~d}, J=8.9 \mathrm{~Hz}, 1 \mathrm{H}), 7.47(\mathrm{dd}, J=13.4,7.6 \mathrm{~Hz}, 1 \mathrm{H})$, $7.33(\mathrm{t}, J=7.3 \mathrm{~Hz}, 1 \mathrm{H}) .{ }^{19} \mathrm{~F}$ NMR $\left(376 \mathrm{MHz}, \mathrm{CDCl}_{3}\right) \delta-112.00,-112.02,-112.04$, -112.06. ${ }^{13} \mathrm{C}$ NMR $\left(101 \mathrm{MHz}, \mathrm{CDCl}_{3}\right) \delta 171.20,163.75,161.29,130.21,130.14$, $125.97,125.94,121.10,120.89,117.16,116.93$. Spectral data matched those previously reported. ${ }^{9}$<smiles>O=C(O)c1cc(F)cc(P)c1</smiles>

$\mathbf{2} \mathbf{j}$ was obtained as a white solid starting from $\mathbf{1 j}$ (106 $\mathrm{mg}, 0.5 \mathrm{mmol})$ according to the General Procedure III in $87 \%$ yield $(68.6 \mathrm{mg}) .{ }^{1} \mathrm{H} \mathrm{NMR}\left(400 \mathrm{MHz}, \mathrm{CDCl}_{3}\right) \delta 12.54(\mathrm{~s}$, $1 \mathrm{H}), 7.62(\mathrm{~d}, J=4.7 \mathrm{~Hz}, 2 \mathrm{H}), 7.08(\mathrm{t}, J=7.8 \mathrm{~Hz}, 1 \mathrm{H}) .{ }^{19} \mathrm{~F} \mathrm{NMR}\left(376 \mathrm{MHz}, \mathrm{CDCl}_{3}\right) \delta$ -108.03, -108.05, -108.07. ${ }^{13} \mathrm{C}$ NMR (101 MHz, $\left.\mathrm{CDCl}_{3}\right) \delta 170.21,164.06,163.94$, 161.57, 161.45, 132.37, 132.27, 132.18, 113.37, 113.29, 113.17, 113.10, 109.68, $109.43,109.18$. Spectral data matched those previously reported. ${ }^{10}$<smiles>O=C(O)c1ccc(Cl)cc1</smiles>

$2 \mathrm{k}$

2k was obtained as a white solid starting from $1 \mathbf{k}(105 \mathrm{mg}, 0.5 \mathrm{mmol})$ according to the General Procedure III in $83 \%$ yield $(64.9 \mathrm{mg}) .{ }^{1} \mathrm{H}$ NMR $\left(400 \mathrm{MHz}, \mathrm{DMSO}-\mathrm{d}_{6}\right) \delta$ $13.23(\mathrm{~s}, 1 \mathrm{H}), 7.98(\mathrm{~d}, J=8.0 \mathrm{~Hz}, 2 \mathrm{H}), 7.61(\mathrm{~d}, J=8.4 \mathrm{~Hz}, 2 \mathrm{H}) .{ }^{13} \mathrm{C}$ NMR $(101 \mathrm{MHz}$, DMSO- $\left.\mathrm{d}_{6}\right) \delta 166.48,137.81,131.16,129.63,128.80$. Spectral data matched those 
previously reported. $^{6}$<smiles>CC(=O)c1ccc(C(=O)O)cc1</smiles>

2I

2l was obtained as a white solid starting from 11 (109 $\mathrm{mg}, 0.5 \mathrm{mmol})$ according to the General Procedure III in $87 \%$ yield $(71.2 \mathrm{mg}) .{ }^{1} \mathrm{H}$ NMR (400 MHz, DMSO-d 6 ) $\delta$ $13.34(\mathrm{~s}, 1 \mathrm{H}), 8.09$ (s, 4H), $2.66(\mathrm{~s}, 3 \mathrm{H}) .{ }^{13} \mathrm{C}$ NMR (101 MHz, DMSO-d 6 ) $\delta 197.74$, $166.67,139.83,134.51,129.57,128.35,27.04$. Spectral data matched those previously reported. ${ }^{6}$<smiles>CC(=O)c1ccc(C(=O)O)cc1</smiles>

$2 m$

$\mathbf{2 m}$ was obtained as a white solid starting from $\mathbf{1 m}(117 \mathrm{mg}, 0.5 \mathrm{mmol})$ according to the General Procedure III in 93\% yield $(83.3 \mathrm{mg}) .{ }^{1} \mathrm{H}$ NMR $\left(400 \mathrm{MHz}, \mathrm{DMSO}-\mathrm{d}_{6}\right) \delta$ $13.43(\mathrm{~s}, 1 \mathrm{H}), 8.09$ (s, 4H), 3.92 (s, 3H). ${ }^{13} \mathrm{C}$ NMR (101 MHz, DMSO-d 6 ) $\delta 166.57$, $165.61,134.83,133.14,129.60,129.36,52.49$. Spectral data matched those previously reported. ${ }^{6}$<smiles>O=C(O)c1ccc(C(F)(F)F)cc1</smiles>

2n

2n was obtained as a white solid starting from 1 n $(112 \mathrm{mg}, 0.5 \mathrm{mmol})$ according to the General Procedure III in $91 \%$ yield $(86.4 \mathrm{mg}) .{ }^{1} \mathrm{H}$ NMR $\left(400 \mathrm{MHz}, \mathrm{DMSO}-\mathrm{d}_{6}\right) \delta$ $13.52(\mathrm{~s}, 1 \mathrm{H}), 8.16(\mathrm{~d}, J=5.8 \mathrm{~Hz}, 2 \mathrm{H}), 7.88(\mathrm{t}, J=6.6 \mathrm{~Hz}, 2 \mathrm{H}) .{ }^{13} \mathrm{C} \mathrm{NMR}(101 \mathrm{MHz}$, DMSO-d $\left._{6}\right) \delta 166.80(\mathrm{~s}), 135.18(\mathrm{~s}), 133.10(\mathrm{q}, J=31.6 \mathrm{~Hz}), 131.27-130.22(\mathrm{~m})$, 126.08 (s), 125.73 (s), 123.02 (s). Spectral data matched those previously reported. ${ }^{6}$<smiles>N#Cc1ccc(C(=O)O)cc1</smiles>

20

2o was obtained as a white solid starting from $10(101 \mathrm{mg}, 0.5 \mathrm{mmol})$ according to the General Procedure III in 97\% yield $(71.2 \mathrm{mg}) .{ }^{1} \mathrm{H}$ NMR (400 MHz, DMSO-d 6 ) $\delta$ $13.62(\mathrm{~s}, 1 \mathrm{H}), 8.12(\mathrm{~d}, J=8.2 \mathrm{~Hz}, 2 \mathrm{H}), 8.02(\mathrm{~d}, J=8.1 \mathrm{~Hz}, 2 \mathrm{H}) .{ }^{13} \mathrm{C}$ NMR $(101 \mathrm{MHz}$, DMSO-d $\left.{ }_{6}\right) \delta 165.97,134.78,132.69,129.96,118.20,115.05$. Spectral data matched those previously reported. ${ }^{6}$<smiles>N#Cc1cccc(C(=O)O)c1</smiles> 
2p was obtained as a white solid starting from $1 \mathbf{p}(100.5 \mathrm{mg}, 0.5 \mathrm{mmol})$ according to the General Procedure III in $89 \%$ yield $(65.4 \mathrm{mg}) .{ }^{1} \mathrm{H}$ NMR (400 MHz, DMSO) $\delta 8.31$ $-8.26(\mathrm{~m}, 1 \mathrm{H}), 8.26-8.21(\mathrm{~m}, 1 \mathrm{H}), 8.12-8.06(\mathrm{~m}, 1 \mathrm{H}), 7.73(\mathrm{t}, J=7.8 \mathrm{~Hz}, 1 \mathrm{H}) .{ }^{13} \mathrm{C}$ NMR (101 MHz, DMSO) $\delta 166.31,136.80,134.34,133.35,132.70,130.64,118.67$, 112.50. Spectral data matched those previously reported. ${ }^{9}$<smiles>CS(=O)(=O)c1ccc(C(=O)O)cc1</smiles>

$2 q$

2q was obtained as a white solid starting from $1 \mathbf{1 q}(127 \mathrm{mg}, 0.5 \mathrm{mmol})$ according to the General Procedure III in $81 \%$ yield $(80.8 \mathrm{mg}) .{ }^{1} \mathrm{H}$ NMR $\left(400 \mathrm{MHz}, \mathrm{DMSO}-\mathrm{d}_{6}\right) \delta$ $8.20(\mathrm{~d}, J=7.7 \mathrm{~Hz}, 2 \mathrm{H}), 8.08(\mathrm{~d}, J=8.0 \mathrm{~Hz}, 2 \mathrm{H}), 3.30(\mathrm{~s}, 3 \mathrm{H}) .{ }^{13} \mathrm{C}$ NMR $(101 \mathrm{MHz}$, DMSO-d 6 ) $\delta 166.40,144.39,135.43,130.44,127.53$. Spectral data matched those previously reported. ${ }^{7}$<smiles>O=C(O)c1ccc2ccccc2c1</smiles>

$2 r$

2r was obtained as a white solid starting from $1 \mathbf{r}(113 \mathrm{mg}, 0.5 \mathrm{mmol})$ according to the General Procedure III in 91\% yield (78.4 mg). ${ }^{1} \mathrm{H}$ NMR (400 MHz, DMSO) $\delta 13.12$ (s, $1 \mathrm{H}), 8.63(\mathrm{~s}, 1 \mathrm{H}), 8.14(\mathrm{~d}, J=7.9 \mathrm{~Hz}, 1 \mathrm{H}), 8.02(\mathrm{~d}, J=5.3 \mathrm{~Hz}, 3 \mathrm{H}), 7.65(\mathrm{dd}, J=13.3$, $7.7 \mathrm{~Hz}, 2 \mathrm{H}) .{ }^{13} \mathrm{C}$ NMR (101 MHz, DMSO) $\delta 167.98,135.46,132.68,131.06,129.82$, $128.87,128.71,128.19,127.35,125.69$. Spectral data matched those previously reported. ${ }^{6}$<smiles>O=C(O)c1ccc2c(c1)CCCC2=O</smiles>

2s

$2 \mathrm{~s}$ was obtained as a white solid starting from $1 \mathrm{~s}(122 \mathrm{mg}, 0.5 \mathrm{mmol})$ according to the General Procedure III in 86\% yield (81.5 mg). ${ }^{1} \mathrm{H}$ NMR (400 MHz, DMSO) $\delta 13.31$ (s, $1 \mathrm{H}), 8.19(\mathrm{~d}, J=7.7 \mathrm{~Hz}, 1 \mathrm{H}), 8.12(\mathrm{~d}, J=7.6 \mathrm{~Hz}, 1 \mathrm{H}), 7.55(\mathrm{t}, J=7.7 \mathrm{~Hz}, 1 \mathrm{H}), 3.33$ $(\mathrm{t}, J=5.6 \mathrm{~Hz}, 2 \mathrm{H}), 2.73(\mathrm{t}, J=6.1 \mathrm{~Hz}, 2 \mathrm{H}), 2.20-2.07(\mathrm{~m}, 2 \mathrm{H}) .{ }^{13} \mathrm{C} \mathrm{NMR}(101 \mathrm{MHz}$, DMSO) $\delta 197.68,168.85,145.39,135.27,133.76,132.04,130.43,126.80,38.61$, $27.73,22.80$. Spectral data matched those previously reported. ${ }^{12}$<smiles></smiles>

2t was obtained as a white solid starting from $1 \mathrm{t}$ (147 $\mathrm{mg}, 0.5 \mathrm{mmol})$ according to the General Procedure III in 90\% yield (107.7 mg). ${ }^{1} \mathrm{H}$ NMR (400 MHz, DMSO) $\delta 11.00$ 
(s, 1H), $8.15(\mathrm{~d}, J=7.6 \mathrm{~Hz}, 1 \mathrm{H}), 8.04(\mathrm{~d}, J=8.7 \mathrm{~Hz}, 1 \mathrm{H}), 7.81(\mathrm{t}, J=7.5 \mathrm{~Hz}, 1 \mathrm{H})$, $7.59(\mathrm{~d}, J=8.3 \mathrm{~Hz}, 1 \mathrm{H}), 7.43(\mathrm{t}, J=7.2 \mathrm{~Hz}, 1 \mathrm{H}), 6.92(\mathrm{~d}, J=8.7 \mathrm{~Hz}, 1 \mathrm{H}), 6.88(\mathrm{~s}$, 1H). ${ }^{13} \mathrm{C}$ NMR (101 MHz, DMSO) $\delta 175.30,164.56,158.08,156.08,135.33,128.54$, $126.39,124.65,121.74,118.41,114.68,114.53,102.68$. Spectral data matched those previously reported. ${ }^{14}$<smiles>CCOC(=O)c1c(C)n(C)c2ccc(C(=O)O)cc12</smiles>

$\mathbf{2 u}$ was obtained as a white solid starting from $1 \mathbf{u}(158 \mathrm{mg}, 0.5 \mathrm{mmol})$ according to the General Procedure III in $84 \%$ yield $(109 \mathrm{mg}) .{ }^{1} \mathrm{H}$ NMR (400 MHz, DMSO) $\delta$ $12.63(\mathrm{~s}, 1 \mathrm{H}), 8.67(\mathrm{~s}, 1 \mathrm{H}), 7.81(\mathrm{~d}, J=8.4 \mathrm{~Hz}, 1 \mathrm{H}), 7.58(\mathrm{~d}, J=8.3 \mathrm{~Hz}, 1 \mathrm{H}), 4.33(\mathrm{dd}$, $J=13.0,6.5 \mathrm{~Hz}, 2 \mathrm{H}), 3.74(\mathrm{~s}, 3 \mathrm{H}), 2.73(\mathrm{~s}, 3 \mathrm{H}), 1.38(\mathrm{t}, J=6.4 \mathrm{~Hz}, 3 \mathrm{H}) .{ }^{13} \mathrm{C} \mathrm{NMR}$ (101 MHz, DMSO) $\delta 168.73,165.18,147.85,139.17,126.01,124.19,123.47,110.39$, 104.05, 59.68, 30.46, 15.01, 12.28. HRMS (ESI-TOF) $\mathrm{m} / \mathrm{z}$ Calcd for $\mathrm{C}_{14} \mathrm{H}_{16} \mathrm{NO}_{4}[\mathrm{M}+\mathrm{H}]^{+}$262.1074, found 262.1074.<smiles>Cc1ccccc1C(=O)O</smiles>

$2 \mathbf{v}$ was obtained as a white solid starting from $1 \mathbf{v}(95 \mathrm{mg}, 0.5 \mathrm{mmol})$ according to the General Procedure III in $92 \%$ yield $(62.5 \mathrm{mg}) .{ }^{1} \mathrm{H}$ NMR $\left(400 \mathrm{MHz}, \mathrm{CDCl}_{3}\right) \delta 8.09(\mathrm{~d}$, $J=7.9 \mathrm{~Hz}, 1 \mathrm{H}), 7.47(\mathrm{t}, J=7.4 \mathrm{~Hz}, 1 \mathrm{H}), 7.30(\mathrm{t}, J=7.4 \mathrm{~Hz}, 2 \mathrm{H}), 2.68(\mathrm{~s}, 3 \mathrm{H}) .{ }^{13} \mathrm{C}$ NMR $\left(101 \mathrm{MHz}, \mathrm{CDCl}_{3}\right) \delta 173.87,141.74,133.33,132.28,131.94,128.61,126.21$, 22.52. Spectral data matched those previously reported. ${ }^{11}$<smiles>O=C(O)c1ccccc1F</smiles>

2w was obtained as a white solid starting from $\mathbf{1 w}(97 \mathrm{mg}, 0.5 \mathrm{mmol})$ according to the General Procedure III in $87 \%$ yield $(61 \mathrm{mg}) .{ }^{1} \mathrm{H}$ NMR $\left(400 \mathrm{MHz}, \mathrm{CDCl}_{3}\right) \delta 11.95(\mathrm{~s}$, $1 \mathrm{H}), 8.05(\mathrm{t}, J=7.6 \mathrm{~Hz}, 1 \mathrm{H}), 7.60(\mathrm{dd}, J=12.7,7.4 \mathrm{~Hz}, 1 \mathrm{H}), 7.25(\mathrm{t}, J=7.5 \mathrm{~Hz}, 1 \mathrm{H})$, $7.22-7.15(\mathrm{~m}, 1 \mathrm{H}) .{ }^{19} \mathrm{~F}$ NMR $\left(376 \mathrm{MHz}, \mathrm{CDCl}_{3}\right) \delta-108.05--108.26(\mathrm{~m}) .{ }^{13} \mathrm{C} \mathrm{NMR}$ $\left(101 \mathrm{MHz}, \mathrm{cdcl}_{3}\right) \delta 169.88,163.94,161.33,135.71,135.62,132.77,124.14,124.10$, $117.56,117.47,117.29,117.07$. Spectral data matched those previously reported. ${ }^{9}$<smiles>O=C(O)c1ccccc1-c1ccccc1</smiles>

2x 
2x was obtained as a white solid starting from $\mathbf{1 x}(95 \mathrm{mg}, 0.5 \mathrm{mmol})$ according to the General Procedure III in $87 \%$ yield $(86.1 \mathrm{mg}) .{ }^{1} \mathrm{H}$ NMR $\left(400 \mathrm{MHz}, \mathrm{CDCl}_{3}\right) \delta 7.96(\mathrm{~d}$, $J=7.7 \mathrm{~Hz}, 1 \mathrm{H}), 7.57(\mathrm{t}, J=7.5 \mathrm{~Hz}, 1 \mathrm{H}), 7.47-7.30(\mathrm{~m}, 7 \mathrm{H}) .{ }^{13} \mathrm{C} \mathrm{NMR}(101 \mathrm{MHz}$, $\left.\mathrm{CDCl}_{3}\right) \delta 173.86,143.37,140.98,132.14,131.23,130.72,129.28,128.46,128.09$, 127.37, 127.20. Spectral data matched those previously reported. ${ }^{13}$

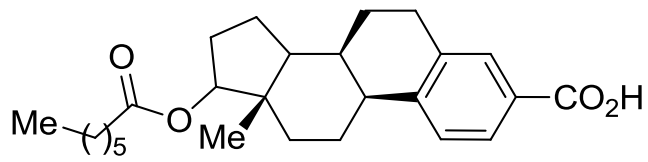

$2 y$

$\mathbf{2 y}$ was obtained as a white solid starting from $\mathbf{1 y}(233.3 \mathrm{mg}, 0.5 \mathrm{mmol})$ according to the General Procedure III in $89 \%$ yield $(365.7 \mathrm{mg}) .{ }^{1} \mathrm{H}$ NMR $\left(400 \mathrm{MHz}, \mathrm{CDCl}_{3}\right)$ $\delta 12.25(\mathrm{~s}, 1 \mathrm{H}), 7.86(\mathrm{~d}, J=8.1 \mathrm{~Hz}, 1 \mathrm{H}), 7.83(\mathrm{~s}, 1 \mathrm{H}), 7.39(\mathrm{~d}, J=8.2 \mathrm{~Hz}, 1 \mathrm{H}), 4.72(\mathrm{t}$, $J=8.4 \mathrm{~Hz}, 1 \mathrm{H}), 2.93(\mathrm{dd}, J=9.2,6.4 \mathrm{~Hz}, 2 \mathrm{H}), 2.40-2.17(\mathrm{~m}, 5 \mathrm{H}), 1.93(\mathrm{dd}, J=15.8$, $7.2 \mathrm{~Hz}, 2 \mathrm{H}), 1.83-1.72(\mathrm{~m}, 1 \mathrm{H}), 1.69-1.23(\mathrm{~m}, 16 \mathrm{H}), 0.89(\mathrm{t}, J=6.5 \mathrm{~Hz}, 3 \mathrm{H}), 0.84$ $(\mathrm{s}, 3 \mathrm{H}) .{ }^{13} \mathrm{C}$ NMR $\left(101 \mathrm{MHz}, \mathrm{CDCl}_{3}\right) \delta 174.37,172.71,147.03,137.36,131.11$, 127.70, 126.88, 125.92, 82.62, 50.27, 44.93, 43.20, 38.30, 37.20, 34.95, 31.81, 29.65, 29.16, 27.89, 27.26, 26.17, 25.43, 23.62, 22.85, 14.39, 12.41. HRMS (ESI-TOF) m/z Calcd for $\mathrm{C}_{26} \mathrm{H}_{37} \mathrm{O}_{4}[\mathrm{M}+\mathrm{H}]^{+}$413.2686, found 413.2684.

\section{General Procedure for the Direct Carboxylation of Aryl Phenols}

General Procedure IV: To a solution of oxime aryl phenols ( $0.5 \mathrm{mmol}, 1.0$ equiv) in DMF (1 mL), NaH (20 mg, $0.5 \mathrm{mmol}, 1.0$ equiv) was added. After stirring for $30 \mathrm{~min}$ at room temperature, the solution was transferred to a $\mathrm{N}_{2}$-filled glovebox. Then the solution was added the $\mathrm{Ni}\left(\mathrm{PPh}_{3}\right)_{2} \mathrm{Cl}_{2}$ (16.3 mg, $0.025 \mathrm{mmol}, 0.05$ equiv), Neocuproine (10.4 mg, $0.05 \mathrm{mmol}, 0.1$ equiv), and $\mathrm{Mn}$ powder ( $82.3 \mathrm{mg}, 1.5 \mathrm{mmol}, 3$ equiv) at the same time. Then the Schlenk tube was transferred out immediately. The solution turned into dark blue. Then the resulting solution was changed into $\mathrm{CO}_{2}$ atmosphere using standard Schlenk techniques and stirred at room temperature for $20 \mathrm{~h}$. After completion of the reaction, the reaction mixture was quenched with $6 \mathrm{M} \mathrm{HCl}$ and extracted with EtOAc (30 mL x 5). The organic layer was collected, dried over $\mathrm{Na}_{2} \mathrm{SO}_{4}$, filtered and concentrated under reduced pressure. The products were purified by column chromatography on silica gel using hexanes/ethyl acetate as the eluent.<smiles>CC(C)(C)c1ccc(C(=O)O)cc1</smiles>

2a was obtained as a white solid starting from 4-(tert-butyl)phenol (116 mg, $0.5 \mathrm{mmol})$ according to the General Procedure IV in $76 \%$ yield $(67.5 \mathrm{mg}) .{ }^{1} \mathrm{H} \mathrm{NMR}$ (400 MHz, $\left.\mathrm{CDCl}_{3}\right) \delta 12.63$ (brs, $\left.1 \mathrm{H}\right), 8.04(\mathrm{~d}, J=7.8 \mathrm{~Hz}, 2 \mathrm{H}), 7.48(\mathrm{~d}, J=7.9 \mathrm{~Hz}, 2 \mathrm{H}), 1.34$ (s, $9 \mathrm{H})$. Spectral data matched those previously reported. ${ }^{6}$ 
<smiles>COc1ccc(C(=O)O)cc1</smiles>

2b

2b was obtained as a white solid starting from 4-methoxyphenol (103 mg, $0.5 \mathrm{mmol})$ according to the General Procedure IV in $79 \%$ yield $(60 \mathrm{mg}) .{ }^{1} \mathrm{H}$ NMR (400 MHz, DMSO-d $\left._{6}\right) \delta 12.64(\mathrm{~s}, 1 \mathrm{H}), 7.90(\mathrm{~d}, J=8.7 \mathrm{~Hz}, 2 \mathrm{H}), 7.01(\mathrm{~d}, J=8.7 \mathrm{~Hz}, 2 \mathrm{H}), 3.82$ (s, $3 \mathrm{H})$. Spectral data matched those previously reported. ${ }^{6}$<smiles>Cc1cc(C)cc(C(=O)O)c1</smiles>

$2 \mathrm{f}$

2f was obtained as a white solid starting from 3,5-dimethylphenol (102 mg, $0.5 \mathrm{mmol})$ according to the General Procedure IV in $77 \%$ yield $(57.3 \mathrm{mg}) .{ }^{1} \mathrm{H}$ NMR $(400 \mathrm{MHz}$, $\left.\mathrm{CDCl}_{3}\right) \delta 12.72(\mathrm{~s}, 1 \mathrm{H}), 7.88(\mathrm{~s}, 1 \mathrm{H}), 7.25(\mathrm{~d}, J=7.9 \mathrm{~Hz}, 1 \mathrm{H}), 7.15(\mathrm{~d}, J=7.5 \mathrm{~Hz}$, $1 \mathrm{H}), 2.60(\mathrm{~s}, 3 \mathrm{H}), 2.35$ (s, 3H). Spectral data matched those previously reported. ${ }^{11}$<smiles>O=C(O)c1ccccc1</smiles>

$2 \mathrm{~g}$

$2 \mathrm{~g}$ was obtained as a white solid starting from phenol ( $88 \mathrm{mg}, 0.5 \mathrm{mmol})$ according to the General Procedure IV in $77 \%$ yield $(46.9 \mathrm{mg}) .{ }^{1} \mathrm{H} \mathrm{NMR}\left(400 \mathrm{MHz}, \mathrm{CDCl}_{3}\right) \delta$ 12.76 (brs, 1H), 8.15 (d, $J=8.0 \mathrm{~Hz}, 2 \mathrm{H}), 7.63$ (t, $J=6.9 \mathrm{~Hz}, 1 \mathrm{H}), 7.49$ (t, $J=7.5 \mathrm{~Hz}$, $2 \mathrm{H})$. Spectral data matched those previously reported. ${ }^{6}$<smiles>O=C(O)c1ccc(-c2ccccc2)cc1</smiles>

$2 \mathrm{~h}$

2h was obtained as a white solid starting from [1,1'-biphenyl]-4-ol (126 mg, $0.5 \mathrm{mmol})$ according to the General Procedure IV in $73 \%$ yield $(72.1 \mathrm{mg}) .{ }^{1} \mathrm{H}$ NMR $(400 \mathrm{MHz}$, DMSO-d $\left._{6}\right) \delta 13.06(\mathrm{~s}, 1 \mathrm{H}), 8.06(\mathrm{~d}, J=8.1 \mathrm{~Hz}, 2 \mathrm{H}), 7.84(\mathrm{~d}, J=8.0 \mathrm{~Hz}, 2 \mathrm{H}), 7.77(\mathrm{~d}$, $J=7.4 \mathrm{~Hz}, 2 \mathrm{H}), 7.54(\mathrm{t}, J=7.4 \mathrm{~Hz}, 2 \mathrm{H}), 7.47(\mathrm{~d}, J=7.2 \mathrm{~Hz}, 1 \mathrm{H})$. Spectral data matched those previously reported. ${ }^{6}$<smiles>O=C(O)c1cccc(F)c1</smiles>

2i

2i was obtained as a white solid starting from 3-fluorophenol (97 $\mathrm{mg}, 0.5 \mathrm{mmol}$ ) according to the General Procedure IV in $73 \%$ yield $(51.2 \mathrm{mg}) .{ }^{1} \mathrm{H}$ NMR (400 MHz, $\left.\mathrm{CDCl}_{3}\right) \delta 11.90(\mathrm{~s}, 1 \mathrm{H}), 7.92(\mathrm{~d}, J=7.5 \mathrm{~Hz}, 1 \mathrm{H}), 7.80(\mathrm{~d}, J=8.9 \mathrm{~Hz}, 1 \mathrm{H}), 7.47(\mathrm{dd}, J$ $=13.4,7.6 \mathrm{~Hz}, 1 \mathrm{H}), 7.33(\mathrm{t}, J=7.3 \mathrm{~Hz}, 1 \mathrm{H})$. Spectral data matched those previously reported. ${ }^{9}$ 
<smiles>O=C(O)c1cc(F)cc(P)c1</smiles>

2j was obtained as a white solid starting from 3,5-difluorophenol (106 mg, $0.5 \mathrm{mmol}$ ) according to the General Procedure IV in $76 \%$ yield $(59.7 \mathrm{mg}) .{ }^{1} \mathrm{H}$ NMR (400 MHz, $\left.\mathrm{CDCl}_{3}\right) \delta 12.54(\mathrm{~s}, 1 \mathrm{H}), 7.62(\mathrm{~d}, J=4.7 \mathrm{~Hz}, 2 \mathrm{H}), 7.08(\mathrm{t}, J=7.8 \mathrm{~Hz}, 1 \mathrm{H})$. Spectral data matched those previously reported. ${ }^{10}$<smiles>CC(=O)c1ccc(C(=O)O)cc1</smiles>

$2 m$

2m was obtained as a white solid starting from methyl 4-hydroxybenzoate (117 mg, $0.5 \mathrm{mmol}$ ) according to the General Procedure IV in $69 \%$ yield $(62 \mathrm{mg}) .{ }^{1} \mathrm{H}$ NMR $\left(400 \mathrm{MHz}, \mathrm{DMSO}_{6}\right) \delta 13.43(\mathrm{~s}, 1 \mathrm{H}), 8.09(\mathrm{~s}, 4 \mathrm{H}), 3.92(\mathrm{~s}, 3 \mathrm{H})$. Spectral data matched those previously reported. ${ }^{6}$<smiles>N#Cc1cccc(C(=O)O)c1</smiles>

$2 p$

2p was obtained as a white solid starting from 3-hydroxybenzonitrile (100.5 mg, 0.5 mmol) according to the General Procedure IV in $62 \%$ yield $(45.5 \mathrm{mg}) .{ }^{1} \mathrm{H}$ NMR $(400$ MHz, DMSO) $\delta 8.31-8.26(\mathrm{~m}, 1 \mathrm{H}), 8.26-8.21(\mathrm{~m}, 1 \mathrm{H}), 8.12-8.06(\mathrm{~m}, 1 \mathrm{H}), 7.73(\mathrm{t}$, $J=7.8 \mathrm{~Hz}, 1 \mathrm{H})$. Spectral data matched those previously reported. ${ }^{9}$<smiles>Cc1ccccc1C(=O)O</smiles>

$2 \mathbf{v}$ was obtained as a white solid starting from $o$-cresol $(95 \mathrm{mg}, 0.5 \mathrm{mmol}$ ) according to the General Procedure IV in $69 \%$ yield $(46.8 \mathrm{mg}) .{ }^{1} \mathrm{H}$ NMR $\left(400 \mathrm{MHz}, \mathrm{CDCl}_{3}\right) \delta$ 8.09 (d, $J=7.9 \mathrm{~Hz}, 1 \mathrm{H}), 7.47$ (t, $J=7.4 \mathrm{~Hz}, 1 \mathrm{H}), 7.30$ (t, $J=7.4 \mathrm{~Hz}, 2 \mathrm{H}), 2.68$ (s, 3H). Spectral data matched those previously reported. ${ }^{11}$<smiles>O=C(O)c1ccccc1F</smiles>

2w

2w was obtained as a white solid starting from 2-fluorophenol $(97 \mathrm{mg}, 0.5 \mathrm{mmol}$ ) according to the General Procedure IV in $72 \%$ yield $(54 \mathrm{mg}) .{ }^{1} \mathrm{H}$ NMR $(400 \mathrm{MHz}$, $\left.\mathrm{CDCl}_{3}\right) \delta 11.95(\mathrm{~s}, 1 \mathrm{H}), 8.05(\mathrm{t}, J=7.6 \mathrm{~Hz}, 1 \mathrm{H}), 7.60(\mathrm{dd}, J=12.7,7.4 \mathrm{~Hz}, 1 \mathrm{H}), 7.25$ $(\mathrm{t}, J=7.5 \mathrm{~Hz}, 1 \mathrm{H}), 7.22-7.15(\mathrm{~m}, 1 \mathrm{H})$. Spectral data matched those previously 
reported. ${ }^{9}$<smiles>O=C(O)c1ccccc1-c1ccccc1</smiles>

2x

$\mathbf{2 x}$ was obtained as a white solid starting from [1,1'-biphenyl]-2-ol (95 mg, $0.5 \mathrm{mmol})$ according to the General Procedure IV in $60 \%$ yield $(59 \mathrm{mg}) .{ }^{1} \mathrm{H}$ NMR (400 MHz, $\left.\mathrm{CDCl}_{3}\right) \delta 7.96(\mathrm{~d}, J=7.7 \mathrm{~Hz}, 1 \mathrm{H}), 7.57(\mathrm{t}, J=7.5 \mathrm{~Hz}, 1 \mathrm{H}), 7.47-7.30(\mathrm{~m}, 7 \mathrm{H})$. Spectral data matched those previously reported. ${ }^{13}$

\section{General Procedure for the Carboxylation of Pyridinyl}

\section{Fluorosulfates}

General Procedure V: All operations were carried out under standard Schlenk and glovebox techniques. In a $\mathrm{N}_{2}$-filled glovebox, to a $50 \mathrm{~mL}$ Schlenk tube with a stir bar was added the $\mathrm{Ni}\left(\mathrm{PPh}_{3}\right)_{2} \mathrm{Cl}_{2}$ (16.3 $\mathrm{mg}, 0.025 \mathrm{mmol}, 0.05$ equiv), Neocuproine (10.4 $\mathrm{mg}, 0.05 \mathrm{mmol}, 0.1$ equiv), and $\mathrm{Mn}$ powder ( $82.3 \mathrm{mg}, 1.5 \mathrm{mmol}, 3$ equiv). Then the Schlenk tube was stirred and evacuated under the vacuum pump for 30 minutes. Then $\mathrm{CO}_{2}$ gas was introduced into the stirring reaction mixture for three times. After that DMF $(0.5 \mathrm{~mL})$ was injected into the tube under the $\mathrm{CO}_{2}$ atmosphere. Then the reaction mixture was stirred for 10 minutes and the mixture was gradually turned into dark blue. Then pyridinyl fluorosulfates $(0.5 \mathrm{mmol})$ and another DMF $(0.5 \mathrm{~mL})$ were introduced into the Schlenk tube under the $\mathrm{CO}_{2}$ atmosphere. Then the resulting solution was stirred at room temperature for $20 \mathrm{~h}$. After completion of the reaction, the reaction mixture was quenched with $6 \mathrm{M} \mathrm{NaOH}$. The solution was filtered. The filtrate was neutralized with $6 \mathrm{M} \mathrm{HCl}$ to $\mathrm{PH}=2$. Then the solution was evaporated to dryness and $\mathrm{MeOH}(15 \mathrm{~mL})$ was added. Then excess $\mathrm{CH}_{2} \mathrm{~N}_{2}$ in ether was slowly dropped into the solution until the solution turn into light yellow. Caution: $\mathrm{CH}_{2} \mathrm{~N}_{2}$ was poisonous. Then the solution was evaporated and extracted with EtOAc (30 mL x 5). The organic layer was collected, dried over $\mathrm{Na}_{2} \mathrm{SO}_{4}$, filtered and concentrated under reduced pressure. The products were purified by column chromatography on silica gel using hexanes/ethyl acetate as the eluent.

\section{Characterization Data for the Carboxylative Products of Pyridinyl}

\section{Fluorosulfates}<smiles>COc1cc(C(C)=O)ccn1</smiles>

$5 a$

5a was obtained as a colorless oil starting from 4a (104 $\mathrm{mg}, 0.5 \mathrm{mmol})$ according to 
the General Procedure V in 67\% yield (55.8 mg). ${ }^{1} \mathrm{H}$ NMR $\left(400 \mathrm{MHz}, \mathrm{CDCl}_{3}\right) \delta 8.26$ $(\mathrm{d}, J=5.1 \mathrm{~Hz}, 1 \mathrm{H}), 7.38(\mathrm{~d}, J=4.9 \mathrm{~Hz}, 1 \mathrm{H}), 7.29(\mathrm{~s}, 1 \mathrm{H}), 3.95(\mathrm{~s}, 3 \mathrm{H}), 3.92(\mathrm{~s}, 3 \mathrm{H})$. ${ }^{13} \mathrm{C}$ NMR $\left(101 \mathrm{MHz}, \mathrm{CDCl}_{3}\right) \delta 165.49,164.76,147.65,140.01,115.65,111.18,77.32$, $77.00,76.68,53.79,52.59$. Spectral data matched those previously reported. ${ }^{21}$<smiles>COc1ccc(C(C)=O)cn1</smiles>

5b

5b was obtained as a colorless oil starting from $4 \mathbf{b}$ (104 $\mathrm{mg}, 0.5 \mathrm{mmol})$ according to the General Procedure V in $79 \%$ yield $(66.1 \mathrm{mg}) .{ }^{1} \mathrm{H}$ NMR $\left(400 \mathrm{MHz}, \mathrm{CDCl}_{3}\right) \delta 8.80$ $(\mathrm{s}, 1 \mathrm{H}), 8.15-8.07(\mathrm{~m}, 1 \mathrm{H}), 6.73(\mathrm{~d}, J=8.7 \mathrm{~Hz}, 1 \mathrm{H}), 3.96(\mathrm{~s}, 3 \mathrm{H}), 3.88(\mathrm{~s}, 3 \mathrm{H}) .{ }^{13} \mathrm{C}$ NMR $\left(101 \mathrm{MHz}, \mathrm{CDCl}_{3}\right) \delta 166.76,165.87,149.97,139.43,119.56,110.60,53.95$, 51.97. Spectral data matched those previously reported. ${ }^{18}$<smiles>COc1cncc(C(C)=O)c1</smiles>

$5 c$

5c was obtained as a colorless oil starting from $4 \mathbf{c}(104 \mathrm{mg}, 0.5 \mathrm{mmol})$ according to the General Procedure V in $83 \%$ yield $(69 \mathrm{mg}) .{ }^{1} \mathrm{H}$ NMR $\left(400 \mathrm{MHz}, \mathrm{CDCl}_{3}\right) \delta 8.78(\mathrm{~s}$, $1 \mathrm{H}), 8.43(\mathrm{~s}, 1 \mathrm{H}), 7.72(\mathrm{~s}, 1 \mathrm{H}), 3.91(\mathrm{~s}, 3 \mathrm{H}), 3.86$ (s, 3H). ${ }^{13} \mathrm{C} \mathrm{NMR}\left(101 \mathrm{MHz}, \mathrm{CDCl}_{3}\right)$ $\delta 165.79,155.42,142.90,142.41,126.36,119.88,55.70,52.47$. Spectral data matched those previously reported. ${ }^{17}$<smiles>COC(=O)c1ccc(C)nc1</smiles>

$5 d$

5d was obtained as a colorless oil starting from $4 \mathbf{d}(96 \mathrm{mg}, 0.5 \mathrm{mmol})$ according to the General Procedure V in $72 \%$ yield $(54.2 \mathrm{mg}) .{ }^{1} \mathrm{H} \mathrm{NMR}\left(400 \mathrm{MHz}, \mathrm{CDCl}_{3}\right) \delta 9.04$ (s, 1H), 8.12 (dd, $J=8.0,1.6 \mathrm{~Hz}, 1 \mathrm{H}), 7.19(\mathrm{~d}, J=8.1 \mathrm{~Hz}, 1 \mathrm{H}), 3.88(\mathrm{~s}, 3 \mathrm{H}), 2.57$ (s, $3 \mathrm{H}) .{ }^{13} \mathrm{C} \mathrm{NMR}\left(101 \mathrm{MHz}, \mathrm{CDCl}_{3}\right) \delta 165.88,163.05,150.36,137.27,123.27,122.89$, $52.19,24.65$. Spectral data matched those previously reported. ${ }^{19}$<smiles>COC(=O)c1cccnc1C</smiles>

5 e

5e was obtained as a colorless oil starting from 4 e $(96 \mathrm{mg}, 0.5 \mathrm{mmol})$ according to the General Procedure V in $71 \%$ yield $(53.3 \mathrm{mg}) .{ }^{1} \mathrm{H} \mathrm{NMR}\left(400 \mathrm{MHz}, \mathrm{CDCl}_{3}\right) \delta 8.59(\mathrm{~d}, J$ $=3.6 \mathrm{~Hz}, 1 \mathrm{H}), 8.17(\mathrm{~d}, J=7.5 \mathrm{~Hz}, 1 \mathrm{H}), 7.19(\mathrm{dd}, J=7.5,5.0 \mathrm{~Hz}, 1 \mathrm{H}), 3.90(\mathrm{~s}, 3 \mathrm{H})$, $2.82(\mathrm{~s}, 3 \mathrm{H}) .{ }^{13} \mathrm{C}$ NMR $\left(101 \mathrm{MHz}, \mathrm{CDCl}_{3}\right) \delta 166.92,159.84,151.77,138.40,125.29$, $120.85,52.26,24.77$. Spectral data matched those previously reported. ${ }^{15}$ 
<smiles>COC(=O)c1ccc(C)nc1C</smiles>

$5 \mathbf{f}$

5f was obtained as a colorless oil starting from $4 \mathbf{f}(103 \mathrm{mg}, 0.5 \mathrm{mmol})$ according to the General Procedure V in $82 \%$ yield $(67.5 \mathrm{mg}) .{ }^{1} \mathrm{H} \mathrm{NMR}\left(400 \mathrm{MHz}, \mathrm{CDCl}_{3}\right) \delta 7.97$ $(\mathrm{d}, J=7.8 \mathrm{~Hz}, 1 \mathrm{H}), 6.94(\mathrm{~d}, J=7.7 \mathrm{~Hz}, 1 \mathrm{H}), 3.79(\mathrm{~s}, 3 \mathrm{H}), 2.70(\mathrm{~s}, 3 \mathrm{H}), 2.45(\mathrm{~s}, 3 \mathrm{H})$. ${ }^{13} \mathrm{C}$ NMR $\left(101 \mathrm{MHz}, \mathrm{CDCl}_{3}\right) \delta 166.87,161.19,159.38,138.73,122.19,120.40,51.96$, 24.64, 24.50. Spectral data matched those previously reported. ${ }^{15}$<smiles>CCc1nc(C)ccc1C(C)=O</smiles>

$5 \mathrm{~g}$

$5 \mathrm{~g}$ was obtained as a colorless oil starting from $4 \mathrm{~g}(118 \mathrm{mg}, 0.5 \mathrm{mmol})$ according to the General Procedure V in $85 \%$ yield $(75.9 \mathrm{mg}) .{ }^{1} \mathrm{H}$ NMR $\left(400 \mathrm{MHz}, \mathrm{CDCl}_{3}\right) \delta 8.00$ $(\mathrm{d}, J=8.0 \mathrm{~Hz}, 1 \mathrm{H}), 6.99(\mathrm{~d}, J=8.0 \mathrm{~Hz}, 1 \mathrm{H}), 3.85(\mathrm{~s}, 3 \mathrm{H}), 3.09(\mathrm{q}, J=7.5 \mathrm{~Hz}, 2 \mathrm{H})$, $2.52(\mathrm{~s}, 3 \mathrm{H}), 1.23(\mathrm{t}, J=7.5 \mathrm{~Hz}, 3 \mathrm{H}) .{ }^{13} \mathrm{C} \mathrm{NMR}\left(101 \mathrm{MHz}, \mathrm{CDCl}_{3}\right) \delta 167.06,164.37$, 161.38, 138.87, 121.84, 120.37, 52.08, 30.42, 24.68, 14.38. HRMS (ESI-TOF) m/z Calcd for $\mathrm{C}_{10} \mathrm{H}_{14} \mathrm{NO}_{2}[\mathrm{M}+\mathrm{H}]^{+} 180.1019$, found 180.1020 .<smiles>CC(=O)c1cnc2ccccc2c1</smiles>

$5 \mathrm{~h}$

5h was obtained as a white solid starting from $\mathbf{4 h}(114 \mathrm{mg}, 0.5 \mathrm{mmol})$ according to the General Procedure V in $74 \%$ yield $(69.1 \mathrm{mg}) .{ }^{1} \mathrm{H}$ NMR $\left(400 \mathrm{MHz}, \mathrm{CDCl}_{3}\right) \delta 9.37$ $(\mathrm{s}, 1 \mathrm{H}), 8.75(\mathrm{~s}, 1 \mathrm{H}), 8.09(\mathrm{~d}, J=8.4 \mathrm{~Hz}, 1 \mathrm{H}), 7.84(\mathrm{~d}, J=8.0 \mathrm{~Hz}, 1 \mathrm{H}), 7.76(\mathrm{t}, J=7.5$ $\mathrm{Hz}, 1 \mathrm{H}), 7.54(\mathrm{t}, J=7.4 \mathrm{~Hz}, 1 \mathrm{H}), 3.95(\mathrm{~s}, 3 \mathrm{H}) .{ }^{13} \mathrm{C} \mathrm{NMR}\left(101 \mathrm{MHz}, \mathrm{CDCl}_{3}\right) \delta 165.72$, $149.88,149.68,138.67,131.78,129.35,129.03,127.36,126.69,122.83,52.41$. Spectral data matched those previously reported. ${ }^{19}$<smiles>COC(=O)c1cccnc1</smiles>

$5 \mathbf{i}$

$5 \mathbf{i}$ was obtained as a colorless oil starting from $4 \mathbf{i}(88.6 \mathrm{mg}, 0.5 \mathrm{mmol})$ according to the General Procedure V in 63\% yield $(43 \mathrm{mg}) .{ }^{1} \mathrm{H}$ NMR $\left(400 \mathrm{MHz}, \mathrm{CDCl}_{3}\right) \delta 9.15(\mathrm{~d}$, $J=1.0 \mathrm{~Hz}, 1 \mathrm{H}), 8.71(\mathrm{~d}, J=4.7 \mathrm{~Hz}, 1 \mathrm{H}), 8.23(\mathrm{~d}, J=8.0 \mathrm{~Hz}, 1 \mathrm{H}), 7.33(\mathrm{dd}, J=7.9$, $4.9 \mathrm{~Hz}, 1 \mathrm{H}), 3.89$ (s, 3H). ${ }^{13} \mathrm{C}$ NMR $\left(101 \mathrm{MHz}, \mathrm{CDCl}_{3}\right) \delta 165.67,153.36,150.82$, $136.99,125.92,123.26,52.39$. Spectral data matched those previously reported. ${ }^{16}$ 
<smiles>CC(=O)c1cc(C)nc(C)c1</smiles>

$5 \mathbf{j}$

$\mathbf{5 j}$ was obtained as a colorless oil starting from $\mathbf{4 j}$ (103 $\mathrm{mg}, 0.5 \mathrm{mmol}$ ) according to the General Procedure V in $61 \%$ yield $(50.1 \mathrm{mg}) .{ }^{1} \mathrm{H}$ NMR $\left(400 \mathrm{MHz}, \mathrm{CDCl}_{3}\right) \delta 7.50$ $(\mathrm{s}, 2 \mathrm{H}), 3.92(\mathrm{~s}, 3 \mathrm{H}), 2.58(\mathrm{~s}, 6 \mathrm{H}) .{ }^{13} \mathrm{C} \mathrm{NMR}\left(101 \mathrm{MHz}, \mathrm{CDCl}_{3}\right) \delta 166.11,158.81$, $137.80,119.43,52.51,24.45$. Spectral data matched those previously reported. ${ }^{20}$<smiles>COC(=O)c1cccc(C)n1</smiles>

$5 \mathbf{k}$

5k was obtained as a colorless oil starting from $4 \mathbf{k}$ (96 $\mathrm{mg}, 0.5 \mathrm{mmol}$ ) according to the General Procedure V in 53\% yield $(40 \mathrm{mg}) .{ }^{1} \mathrm{H}$ NMR $\left(400 \mathrm{MHz}, \mathrm{CDCl}_{3}\right) \delta 7.79$ (d, $J=7.7 \mathrm{~Hz}, 1 \mathrm{H}), 7.56(\mathrm{t}, J=4.0 \mathrm{~Hz}, 1 \mathrm{H}), 7.19(\mathrm{~d}, J=8.2 \mathrm{~Hz}, 1 \mathrm{H}), 3.91(\mathrm{~s}, 3 \mathrm{H}), 2.49$ $(\mathrm{s}, 3 \mathrm{H}) .{ }^{13} \mathrm{C}$ NMR $\left(101 \mathrm{MHz}, \mathrm{CDCl}_{3}\right) \delta 165.74,158.77,147.23,136.98,126.71$, $122.24,52.70,24.48$. Spectral data matched those previously reported. ${ }^{20}$<smiles>COC(=O)c1ccccn1</smiles>

$5 \mathbf{I}$

5l was obtained as a colorless oil starting from $4 \mathbf{l}(89 \mathrm{mg}, 0.5 \mathrm{mmol})$ according to the General Procedure V in $46 \%$ yield $(29.4 \mathrm{mg}) .{ }^{1} \mathrm{H} \mathrm{NMR}\left(400 \mathrm{MHz}, \mathrm{CDCl}_{3}\right) \delta 8.70(\mathrm{~d}, J$ $=3.7 \mathrm{~Hz}, 1 \mathrm{H}), 8.10(\mathrm{~d}, J=7.8 \mathrm{~Hz}, 1 \mathrm{H}), 7.81(\mathrm{t}, J=7.2 \mathrm{~Hz}, 1 \mathrm{H}), 7.54-7.34(\mathrm{~m}, 1 \mathrm{H})$, $3.96(\mathrm{~s}, 3 \mathrm{H}) .{ }^{13} \mathrm{C} \mathrm{NMR}\left(101 \mathrm{MHz}, \mathrm{CDCl}_{3}\right) \delta 165.62,149.74,147.81,137.01,126.93$, $125.08,52.84$. Spectral data matched those previously reported. ${ }^{16}$

\section{1 mmol Scale Synthesis}<smiles>CC(C)COC(=O)c1ccc(OS(=O)(=O)Oc2ccc(C(C)(C)C)cc2)cc1</smiles>

The operation was carried out under standard Schlenk and glovebox techniques. In a $\mathrm{N}_{2}$-filled glovebox, to a $100 \mathrm{~mL}$ Schlenk tube with a stir bar was added the $\mathrm{Ni}\left(\mathrm{PPh}_{3}\right)_{2} \mathrm{Cl}_{2}$ (32.6 mg, $0.05 \mathrm{mmol}, 0.05$ equiv), Neocuproine (20.8 mg, $0.1 \mathrm{mmol}, 0.1$ equiv), and Mn powder (164 mg, $3 \mathrm{mmol}, 3$ equiv). Then the Schlenk tube was stirred and evacuated under the vacuum pump for 30 minutes. Then $\mathrm{CO}_{2}$ gas was introduced into the stirring reaction mixture for three times. After that DMF $(1 \mathrm{~mL})$ was injected into the tube under the $\mathrm{CO}_{2}$ atmosphere. Then the reaction mixture was stirred for 10 minutes and the mixture was gradually turned into dark blue. Then aryl fluorosulfates 
$1 \mathbf{a}(232 \mathrm{mg}, 1 \mathrm{mmol})$ and another DMF (1 mL) were introduced into the Schlenk tube under the $\mathrm{CO}_{2}$ atmosphere. Then the resulting solution was stirred at room temperature for $20 \mathrm{~h}$. After completion of the reaction, the reaction mixture was quenched with $6 \mathrm{M} \mathrm{HCl}$ and extracted with EtOAc (30 mL x 5). The organic layer was collected, dried over $\mathrm{Na}_{2} \mathrm{SO}_{4}$, filtered and concentrated under reduced pressure. The products were purified by column chromatography on silica gel using hexanes/ethyl acetate as the eluent to afford 2a as a white solid in 93\% yield (165.5 mg).

\section{Hammett plot Analysis}

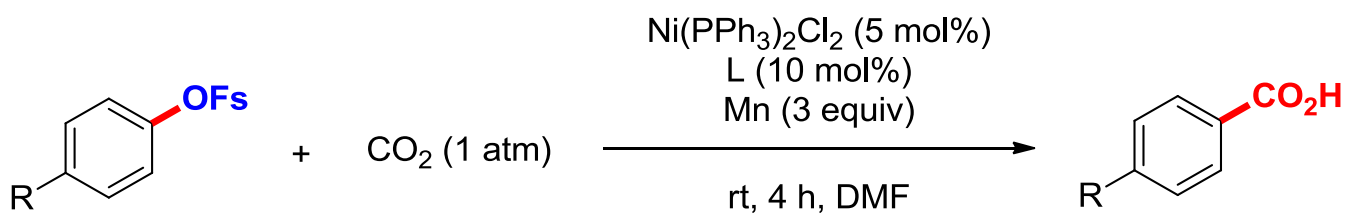

All operations were carried out under standard Schlenk and glovebox techniques. In a $\mathrm{N}_{2}$-filled glovebox, to a $50 \mathrm{~mL}$ Schlenk tube with a stir bar was added the $\mathrm{Ni}\left(\mathrm{PPh}_{3}\right)_{2} \mathrm{Cl}_{2}$ (16.3 mg, $0.025 \mathrm{mmol}, 0.05$ equiv), Neocuproine (10.4 mg, $0.05 \mathrm{mmol}$, 0.1 equiv), and Mn powder ( $82.3 \mathrm{mg}, 1.5 \mathrm{mmol}, 3$ equiv). Then the Schlenk tube was stirred and evacuated under the vacuum pump for 30 minutes. Then $\mathrm{CO}_{2}$ gas was introduced into the stirring reaction mixture for three times. After that DMF $(0.5 \mathrm{~mL})$ was injected into the tube under the $\mathrm{CO}_{2}$ atmosphere. Then the reaction mixture was stirred for 10 minutes and the mixture was gradually turned into dark blue. Then aryl fluorosulfates $(0.5 \mathrm{~mol})$ and another DMF $(0.5 \mathrm{~mL})$ were introduced into the Schlenk tube under the $\mathrm{CO}_{2}$ atmosphere. Then the resulting solution was stirred at room temperature for $4 \mathrm{~h}$. After completion of the reaction, the reaction mixture was quenched with $6 \mathrm{M} \mathrm{HCl}$ and extracted with EtOAc (30 mL x 5). The organic layer was collected, dried over $\mathrm{Na}_{2} \mathrm{SO}_{4}$, filtered and concentrated under reduced pressure. The conversions were determined by ${ }^{1} \mathrm{H}$ NMR with $\mathrm{CH}_{2} \mathrm{Br}_{2}$ as an internal standard.

\begin{tabular}{|c|c|c|c|c|}
\hline entry & Substituent & Conv & $\boldsymbol{\sigma}_{\text {para }}$ & $\log$ (conv.R/conv.H) \\
\hline 1 & $\mathrm{Me}$ & 13.5 & -0.14 & -0.087 \\
\hline 2 & $\mathrm{OMe}$ & 14.5 & -0.12 & -0.056 \\
\hline 3 & $\mathrm{H}$ & 17 & 0 & 0.013 \\
\hline 4 & $\mathrm{Ph}$ & 18 & 0.05 & 0.038 \\
\hline 5 & $\mathrm{COOMe}$ & 23.5 & 0.45 & 0.154 \\
\hline 6 & $\mathrm{CN}$ & 29.5 & 0.70 & 0.252 \\
\hline
\end{tabular}




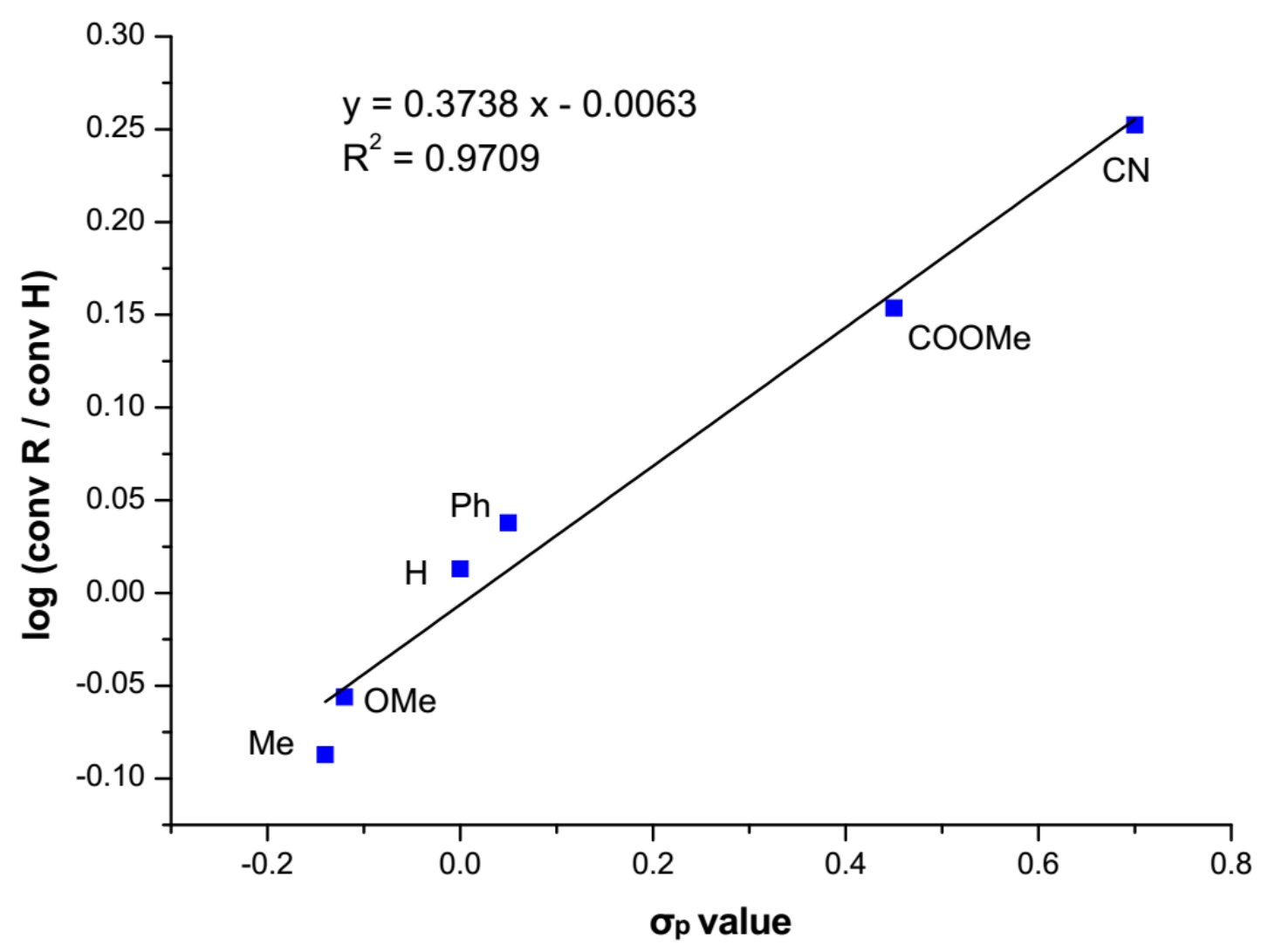

\section{General Procedure for Kinetic Experiments}

Kinetics experiments were run up to about 3-30\% conversion. All operations were carried out under standard Schlenk and glovebox techniques. In a $\mathrm{N}_{2}$-filled glovebox, to a $50 \mathrm{~mL}$ Schlenk tube with a stir bar was added the $\mathrm{Ni}\left(\mathrm{PPh}_{3}\right)_{2} \mathrm{Cl}_{2}(16.3 \mathrm{mg}, 0.025$ mmol, 0.05 equiv), Neocuproine (10.4 mg, $0.05 \mathrm{mmol}, 0.1$ equiv), and $\mathrm{Mn}$ powder ( $82.3 \mathrm{mg}, 1.5 \mathrm{mmol}, 3$ equiv). Then the Schlenk tube was stirred and evacuated under the vacuum pump for 30 minutes. Then $\mathrm{CO}_{2}$ gas was introduced into the stirring reaction mixture for three times. After that DMF $(0.5 \mathrm{~mL})$ was injected into the tube under the $\mathrm{CO}_{2}$ atmosphere. Then the reaction mixture was stirred for 10 minutes and the mixture was gradually turned into dark blue. Then aryl fluorosulfates $(0.5 \mathrm{mmol})$ and another DMF $(0.5 \mathrm{~mL})$ were introduced into the Schlenk tube under the $\mathrm{CO}_{2}$ atmosphere. Then the resulting solution was stirred at room temperature for $2 \mathrm{~h}$. After completion of the reaction, the reaction mixture was quenched with $6 \mathrm{M} \mathrm{HCl}$ and extracted with EtOAc (30 mL x 5). The organic layer was collected, dried over $\mathrm{Na}_{2} \mathrm{SO}_{4}$, filtered and concentrated under reduced pressure. The conversions were determined by ${ }^{1} \mathrm{H} \mathrm{NMR}$ with $\mathrm{CH}_{2} \mathrm{Br}_{2}$ as an internal standard.

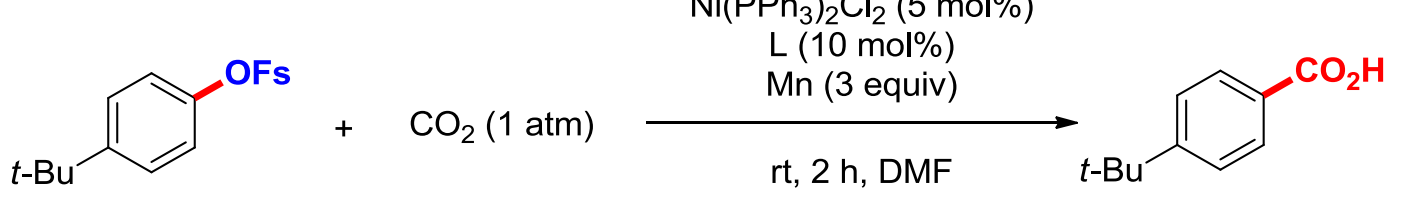




\begin{tabular}{|c|c|c|c|c|}
\hline entry & $\mathbf{1 a}(\mathbf{m m o l})$ & $[\mathbf{1 a}] \mathbf{( m M})$ & Conv 2a $(\boldsymbol{\%})$ & Initial rate $[\mathbf{m M} / \mathbf{h}]$ \\
\hline 1 & 0.1 & 100 & 24 & 12 \\
\hline 2 & 0.2 & 200 & 19 & 9.5 \\
\hline 3 & 0.3 & 300 & 15 & 7.5 \\
\hline 4 & 0.5 & 500 & 13 & 6.5 \\
\hline 5 & 1.5 & 1500 & 4 & 2 \\
\hline
\end{tabular}

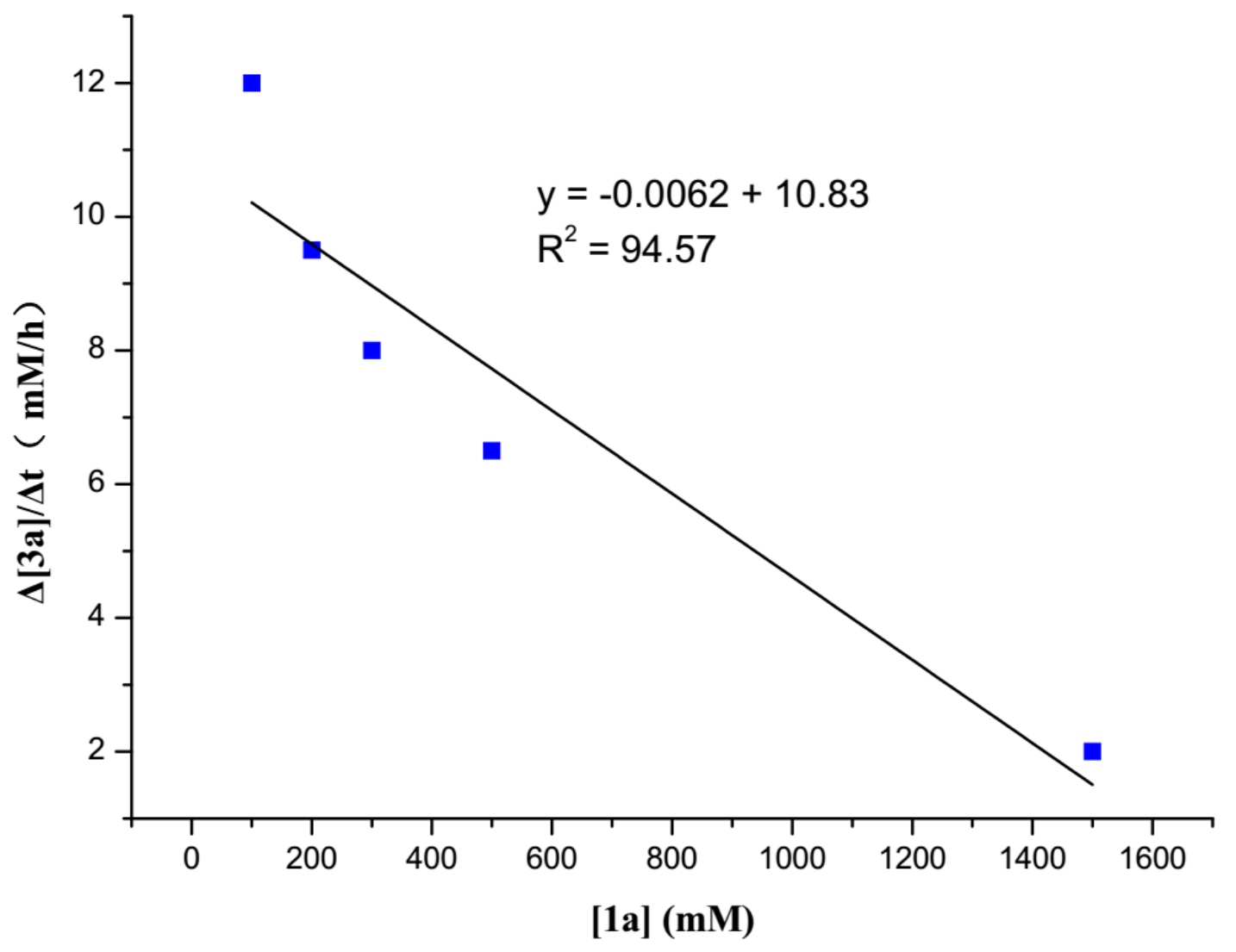




\section{References}

1. Schimler, S. D.; Cismesia, M. A.; Hanley, P. S.; Froese, R. D. J.; Jansma, M. J.; Bland, D. C.; Sanford, M. S. J. Am. Chem. Soc. 2017, 139, 1452.

2. Hanley, P. S.; Ober, M. S.; Krasovskiy, A. L.; Whiteker, G. T.; Kruper, W. J. ACS Catal.2015, 5, 5041.

3. Liang, Q.-B.; Xing, P.; Huang, Z.-G.; Dong, J.-J.; Sharpless, K. B.; Li, X.-X.; Jiang, B. Org. Lett. 2015, 17, 1942.

4. Veryser, C.; Demaerel, J.; Bieliūnas, V.; Gilles, P.; Borggraeve, W. M. D. Org. Lett. 2017, 19, 5244.

5. Zhang, E.; Tang, J.; Li, S.; Wu, P.; Moses, J. E.; Sharpless, K. B. Chem. Eur. J. 2016, 22, 5692.

6. Zhang, X.; Zhang, W.-Z.; Shi, L.-L.; Guo, C.-X.; Zhang, L.-L.; Lu, X.-B. Chem. Commun. 2012, 48, 6292.

7. Joseph, J. T.; Sajith, A. M.; Ningegowda, R. C.; Shashikanth, S. Adv. Synth. Catal. 2017, $359,419$.

8. Sun, S.; Yu, J.-T.; Jiang, Y.; Cheng, J. Adv. Synth. Catal. 2015, 357, 2022.

9. Rebih, F.; Andreini, M.; Moncomble, A.; Harrison-Marchand, A.; Maddaluno, J.; Durandetti, M. Chem. Eur. J. 2016, 22, 3758.

10. Meng, Q.-Y.; Wang, S.; König, B. Angew. Chem. Int. Ed. 2017, 56, 13426.

11. Tran-Vu, H.; Daugulis, O. ACS Catal. 2013, 3, 2417.

12. Aono, T., Araki, Y.; Tanaka, K.; Imanishi, M.; Noguchi, S. Chem. Pharm. Bull. 1978, 26, 1511.

13. Zhu, C.; Zhang, Y.; Kan, J.; Zhao, H.; Su, W.-P. Org. Lett. 2015, 17, 3418.

14. Kuo, C.-W.; Fang, J.-M. Synth. Commun. 2001, 31, 877.

15. Hirai, S.; Horikawa, Y.; Asahara, H.; Nishiwaki, N. Chem. Commun. 2017, 53, 2390.

16. Mane, R. S.; Sasaki, T.; Bhanage, B. M. RSC Adv., 2015, 5, 94776.

17. Buijnsters, P.; Angelis, M. D.; Langlois, X.; Rombouts, F. J. R.; Sanderson, W.; Tresadern, G.; Ritchie, A.; Trabanco, A. A.; Vanhoof, G.; Roosbroeck, Y. V.; Andrés, J.-I. ACS Med. Chem. Lett. 2014, 5, 1049.

18. Kaganovsky, L.; Gelman, D.; Rueck-Braun, K. J. Organomet. Chem. 2010, 695, 260.

19. Jagadeesh, R. V.; Junge, H.; Pohl, M.-M.; Radnik, J.; Brückner, A.; Beller, M. J. Am. Chem. Soc. 2013, 135, 10776.

20. Craig, D.; Henry, G. D. Tetrahedron Lett. 2005, 46, 2559.

21. Subramanyam, C.; Noguchi, M.; Weinreb, S. M. J. Org. Chem., 1989, 54, 5580. 


\section{Copies of NMR Spectrum}
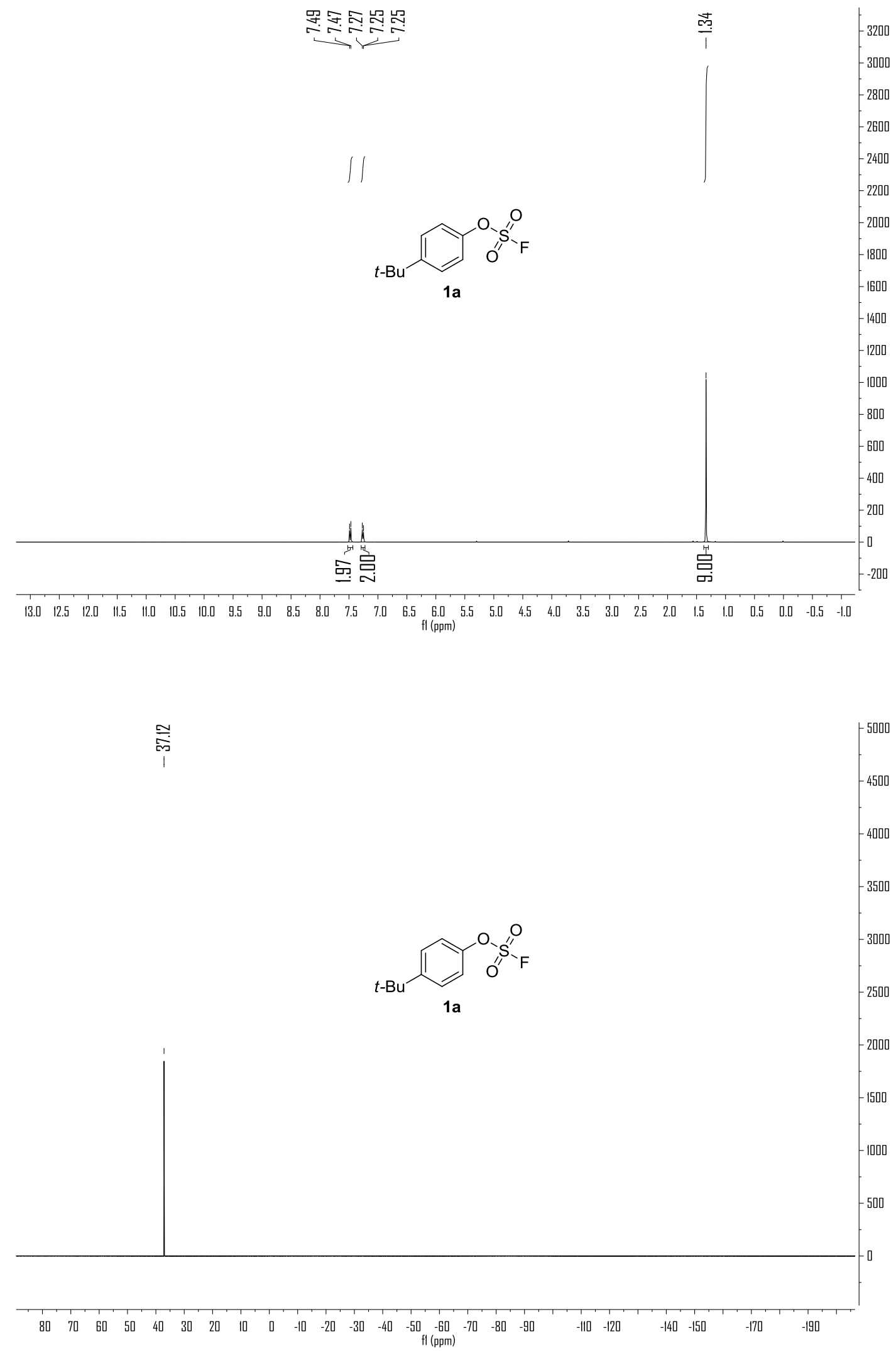

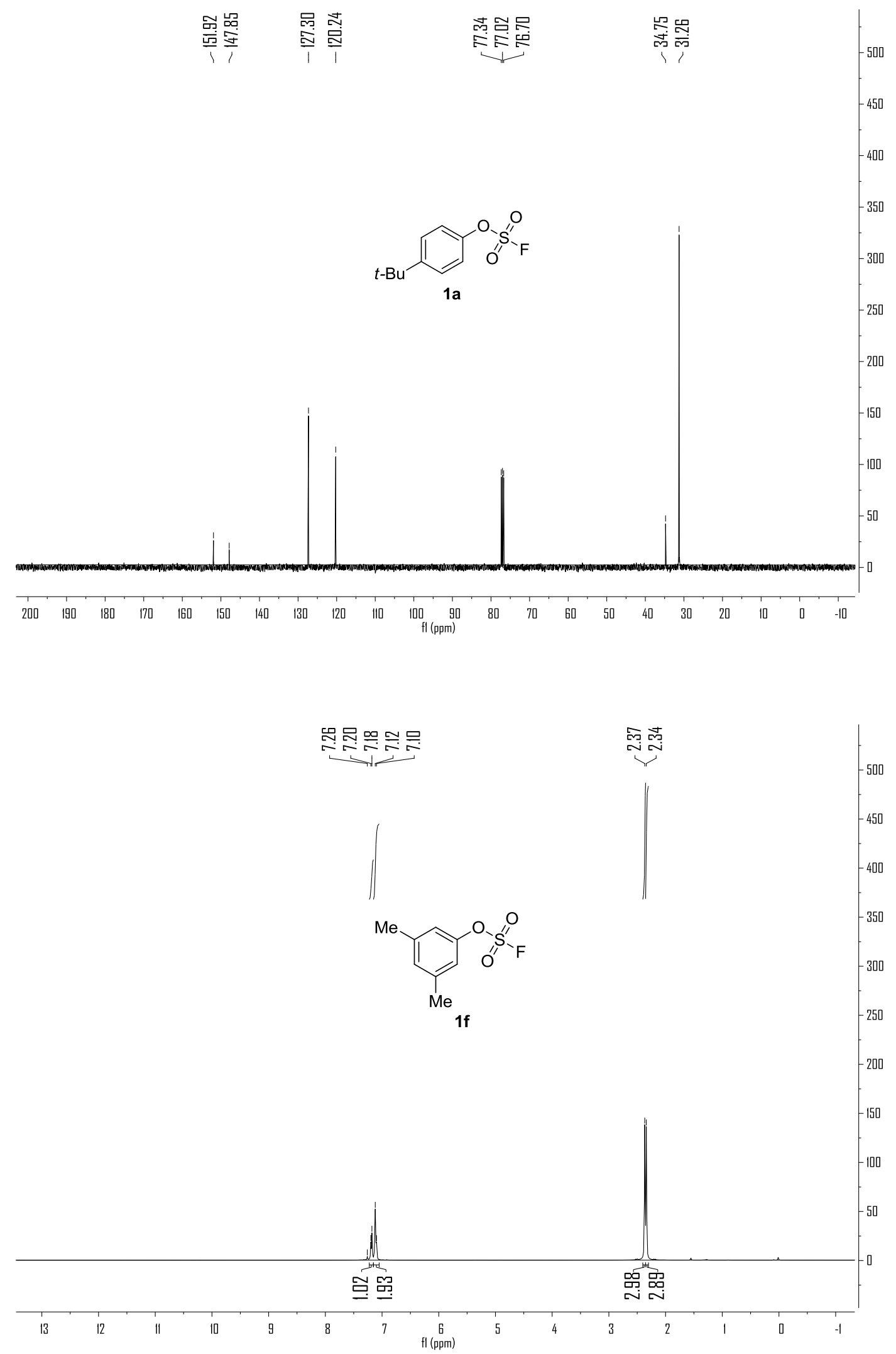

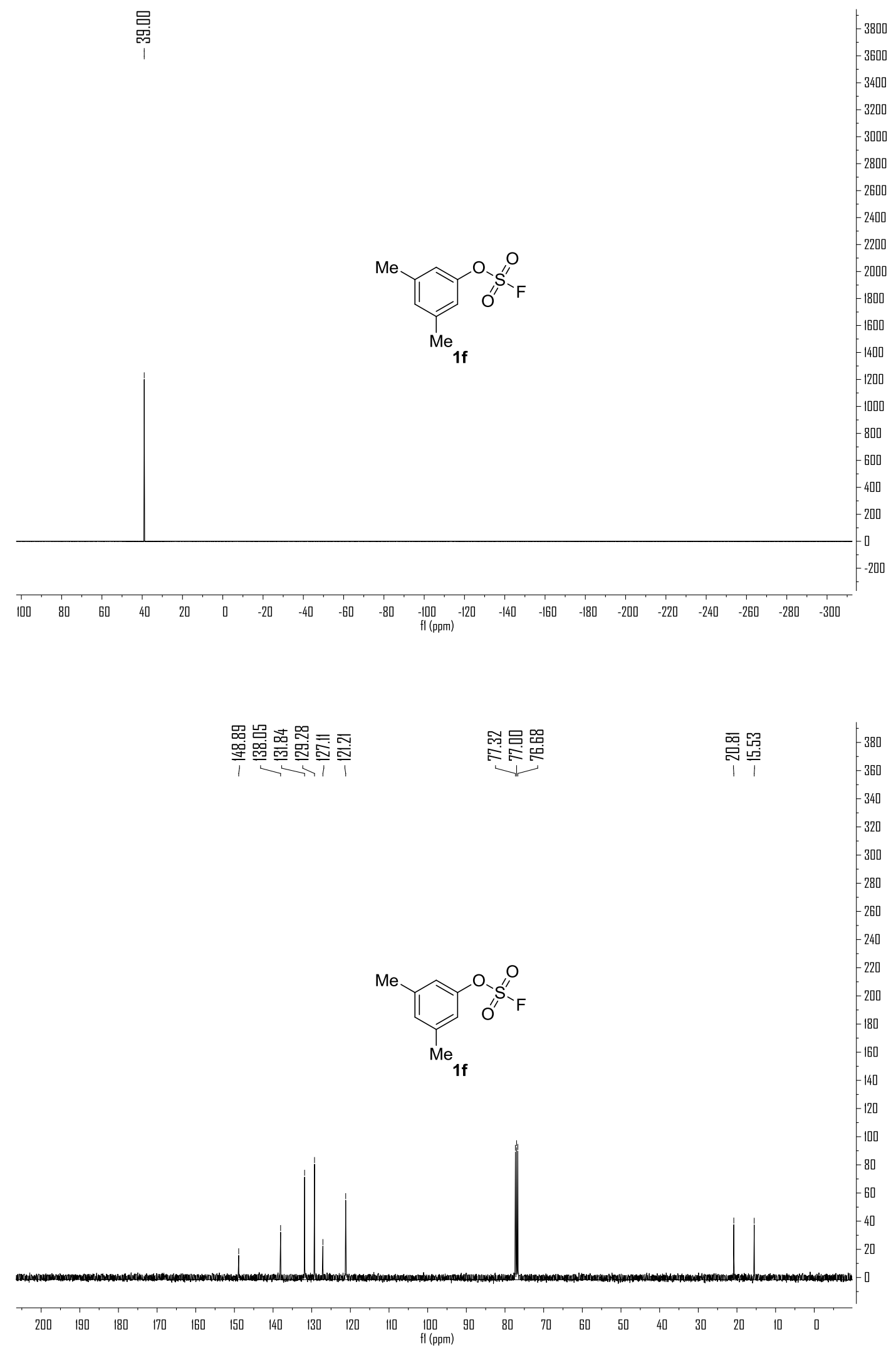
怘总品筥品
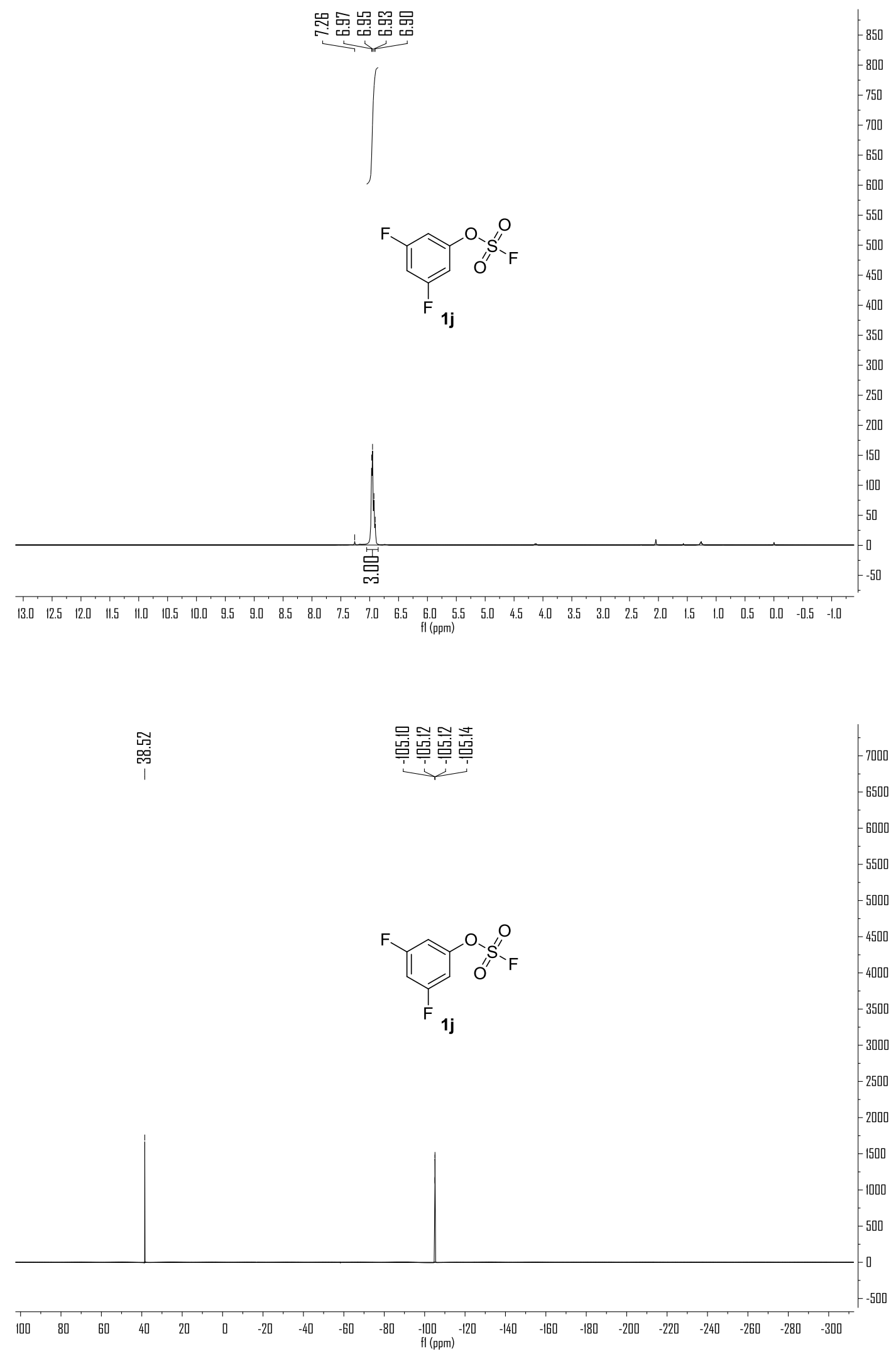

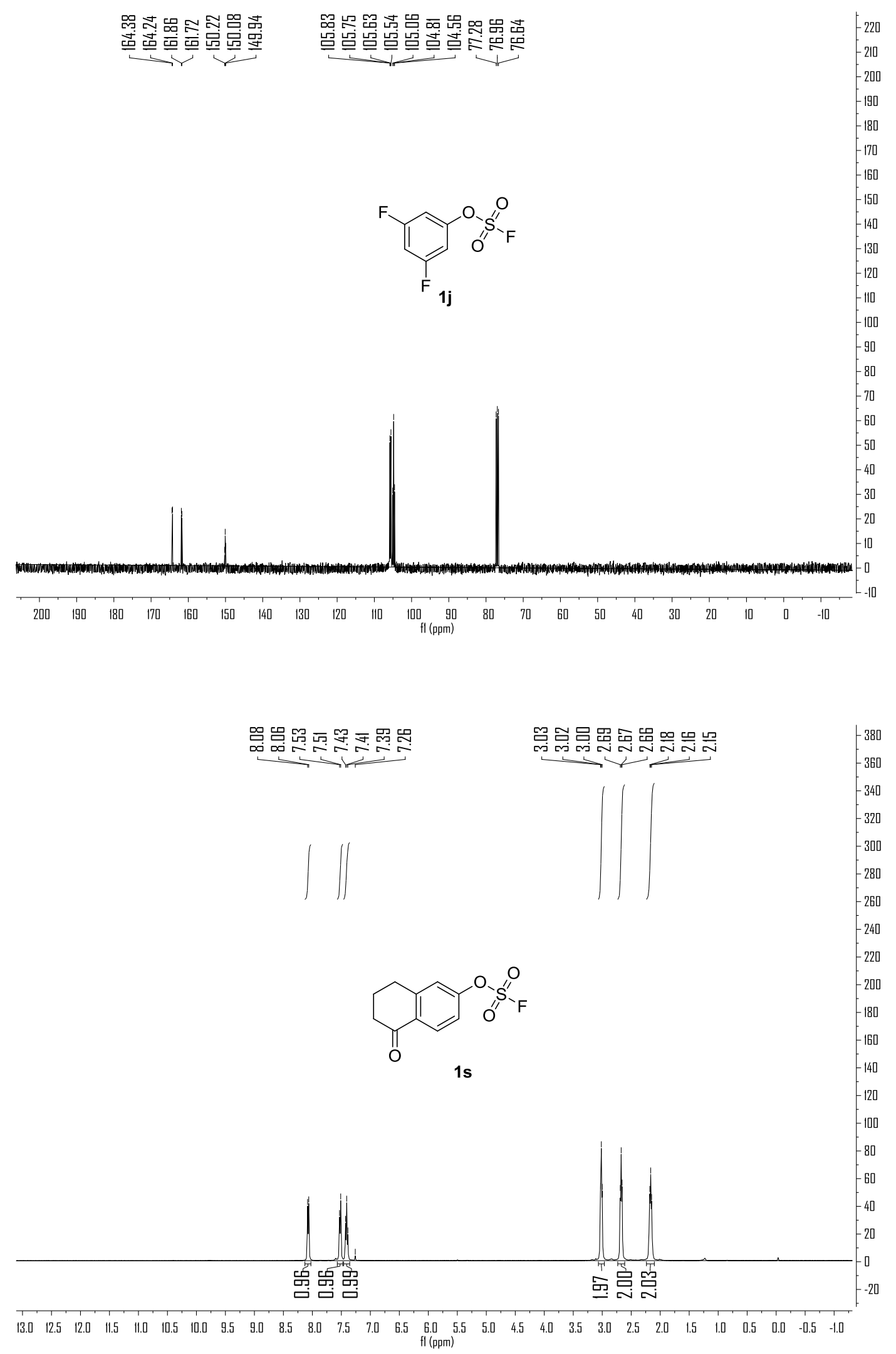

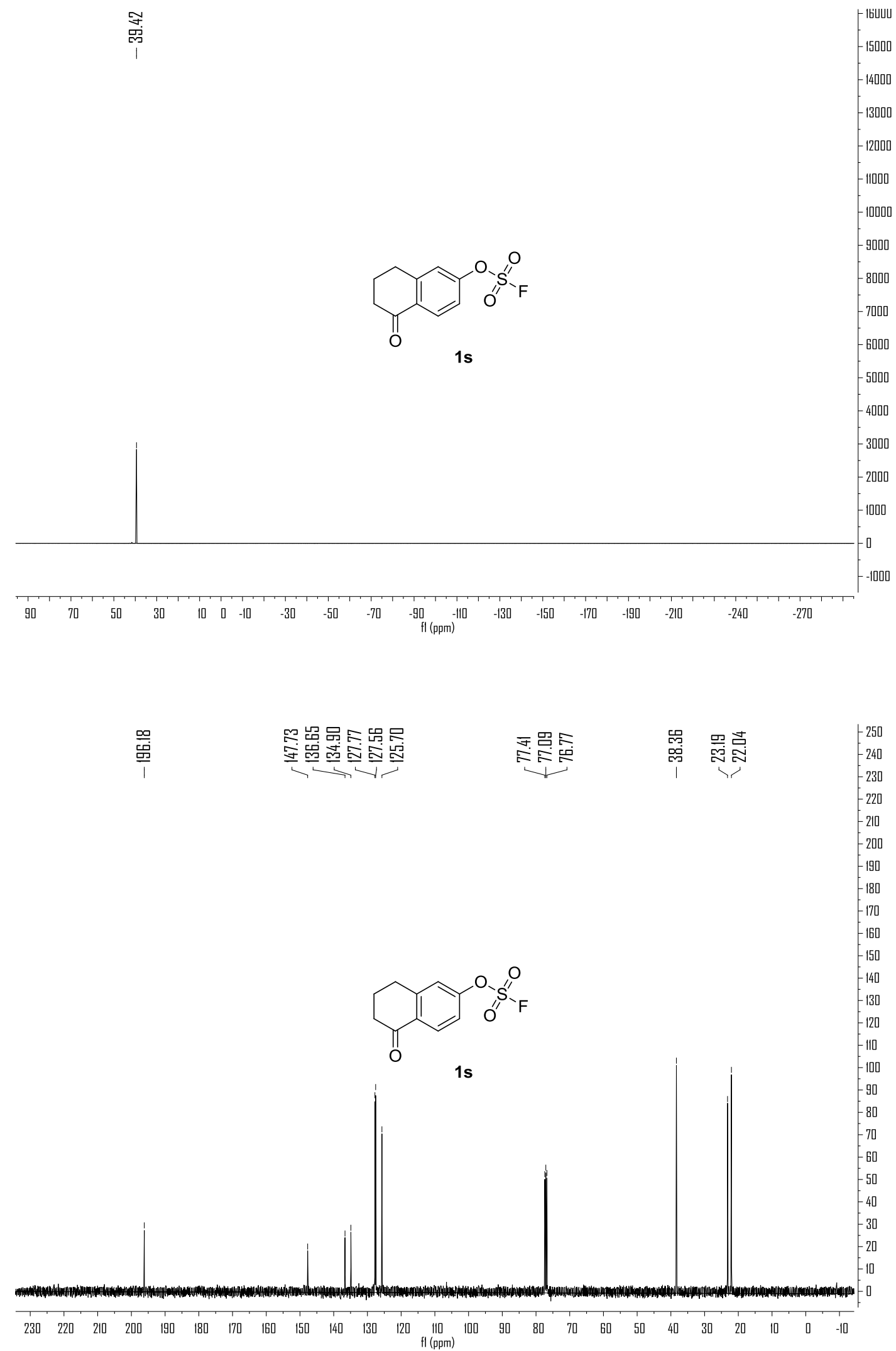


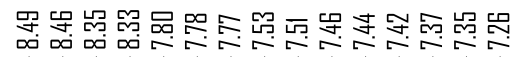
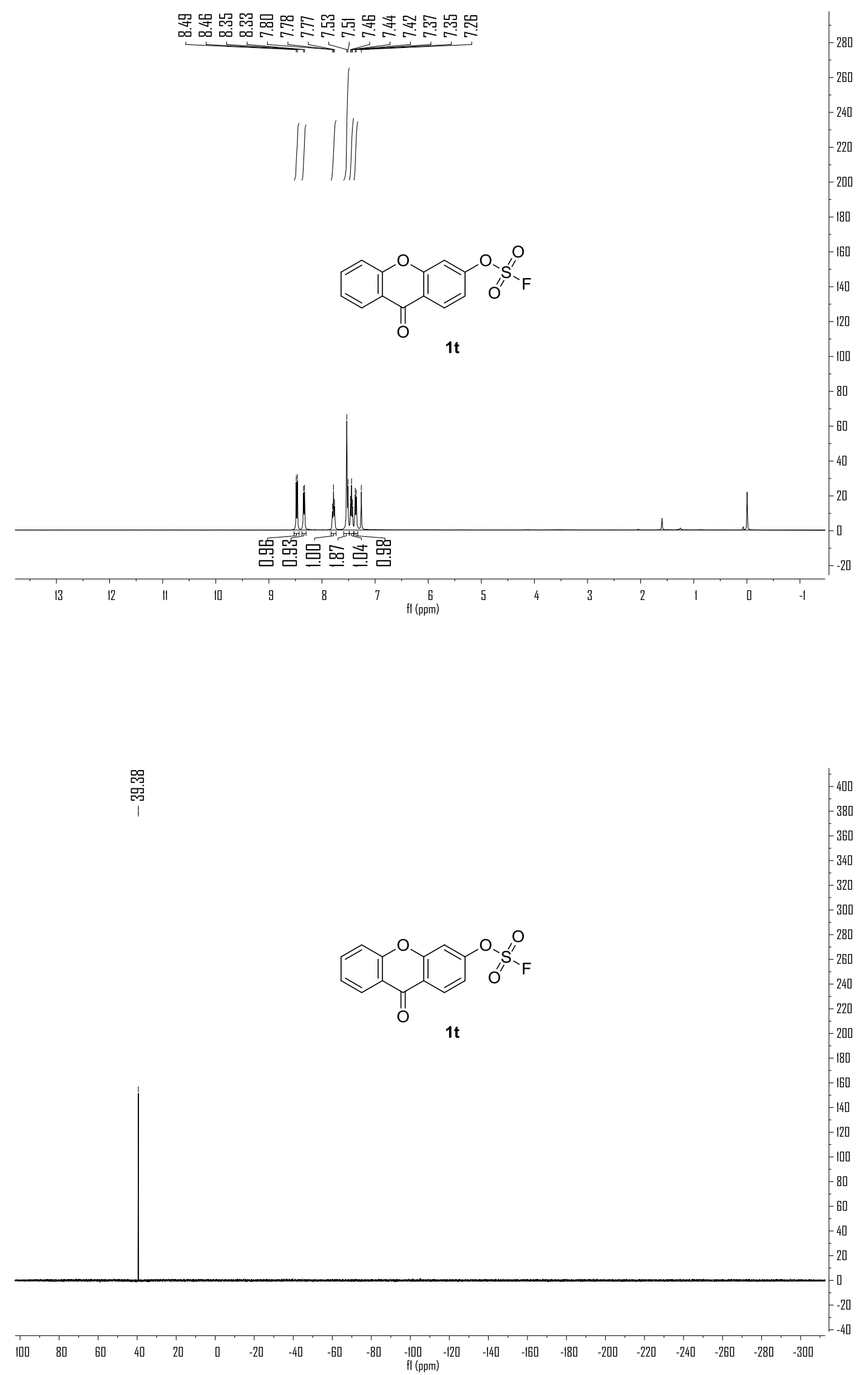

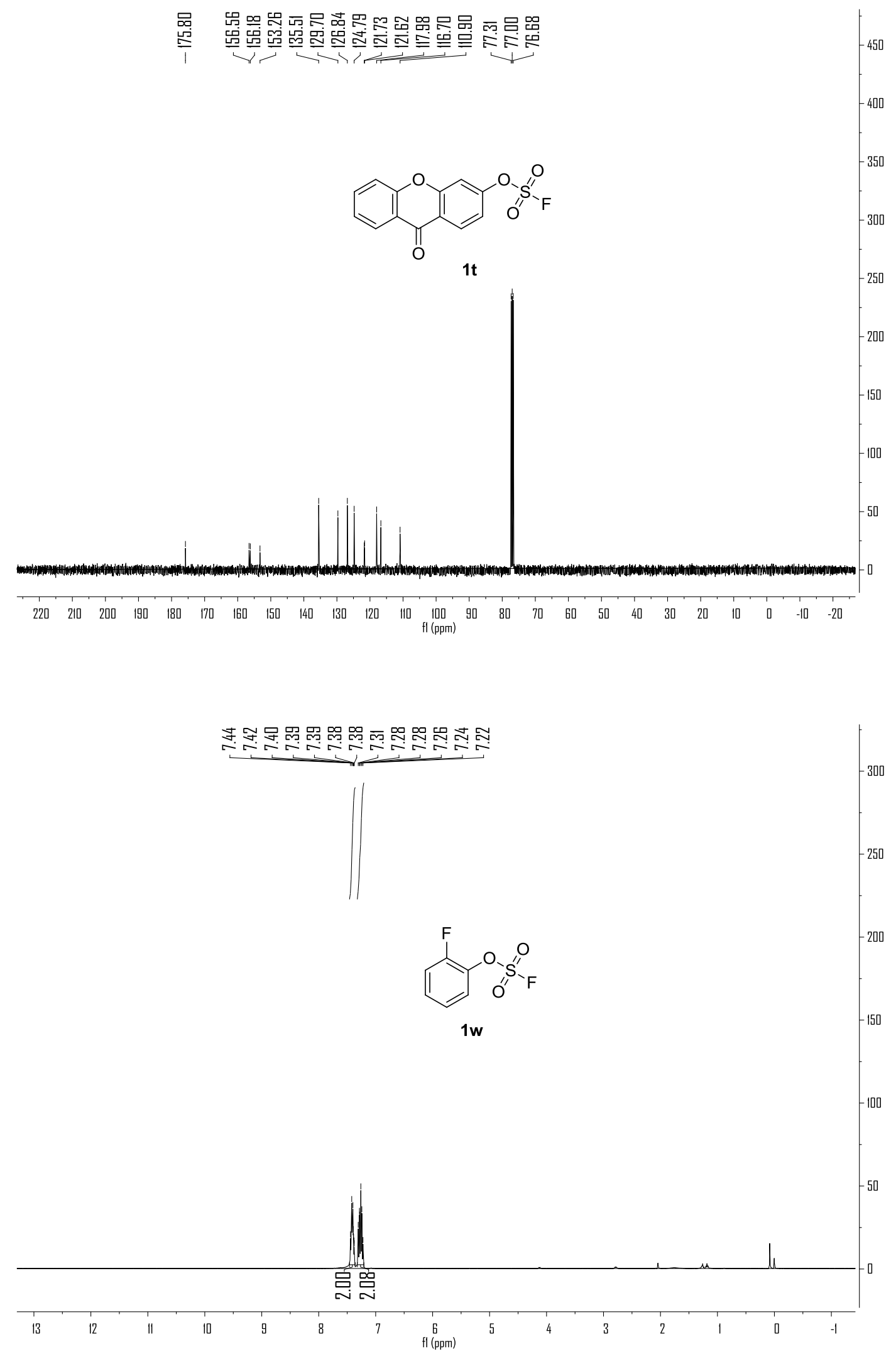

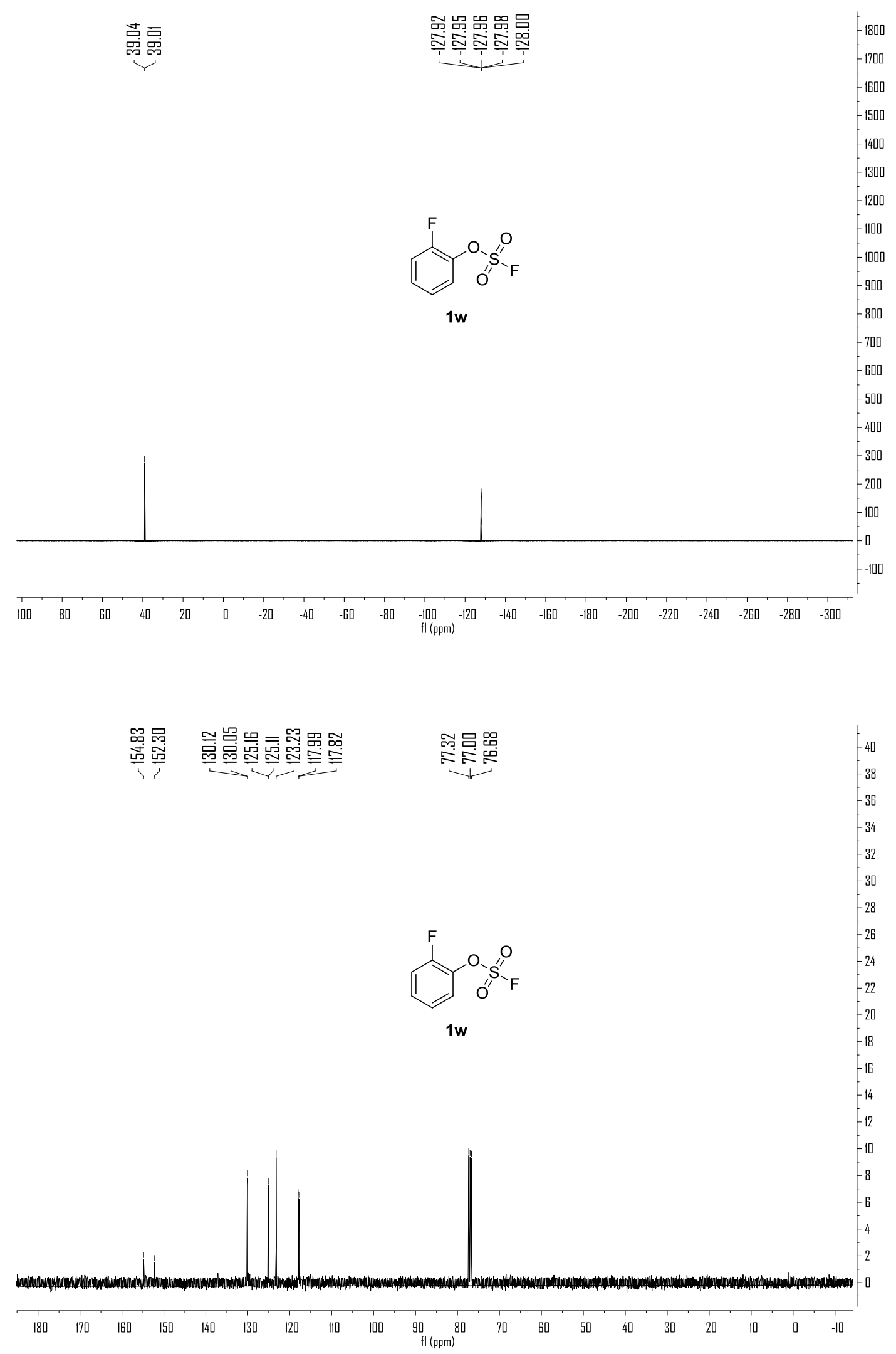

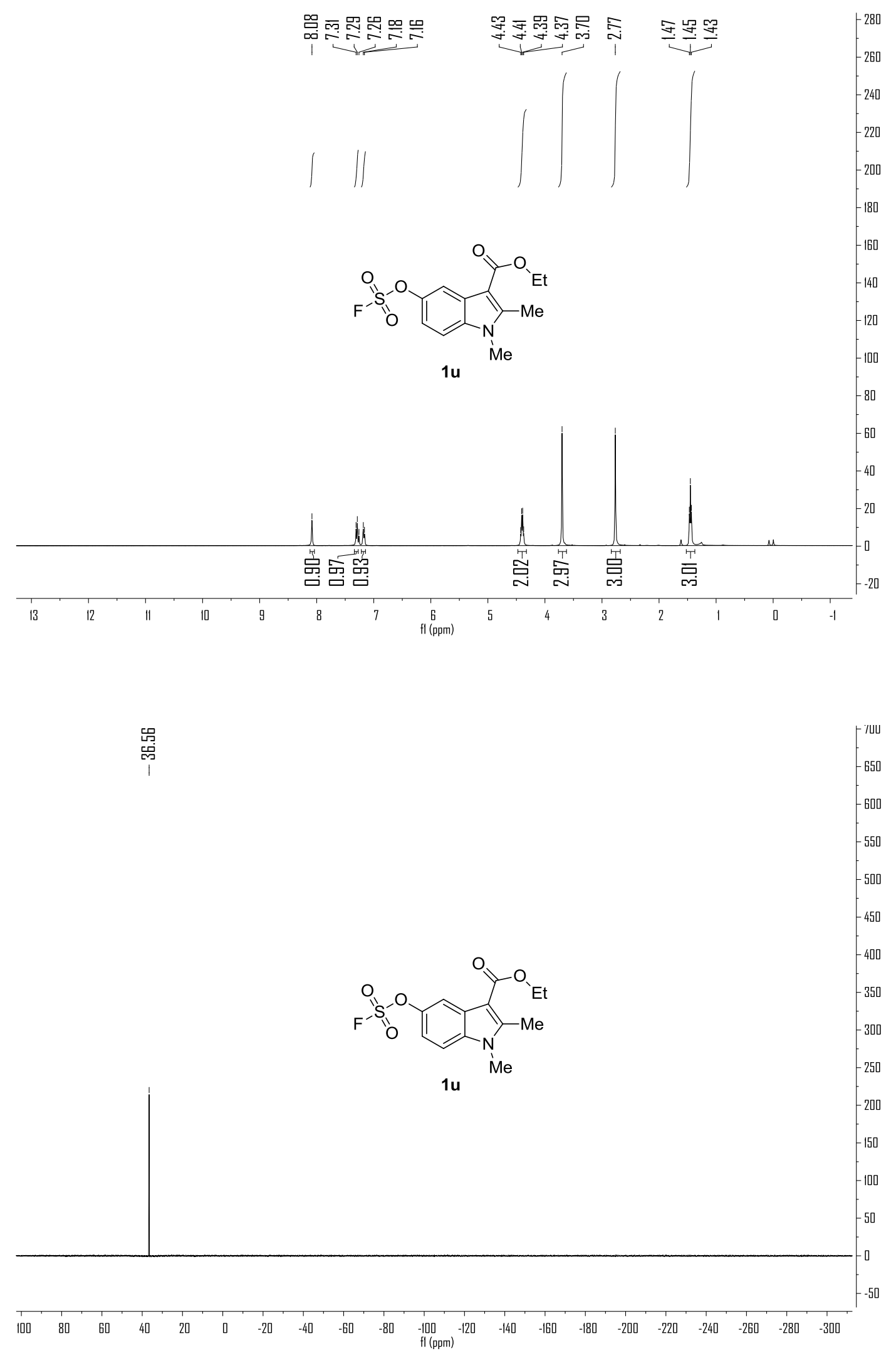


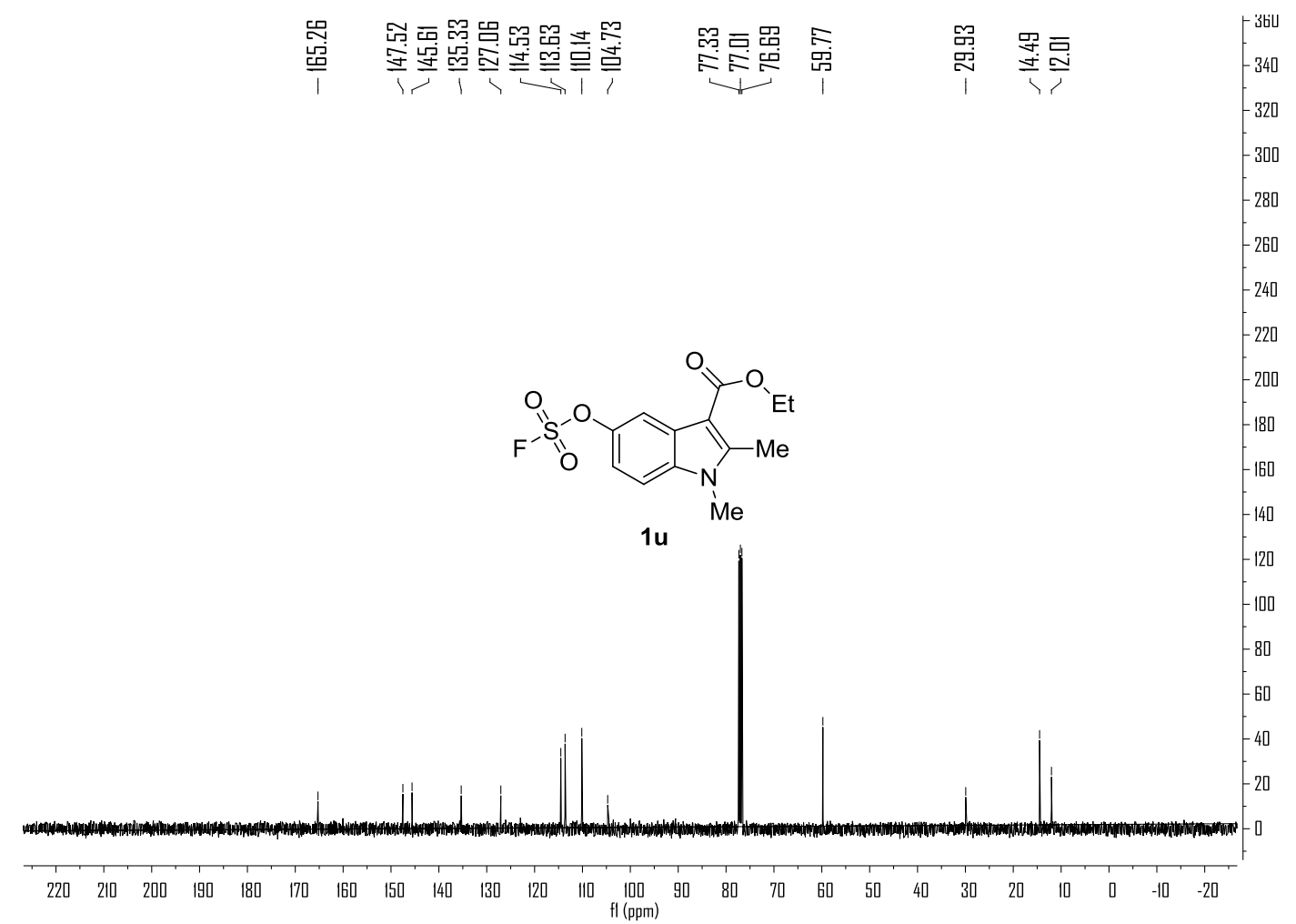

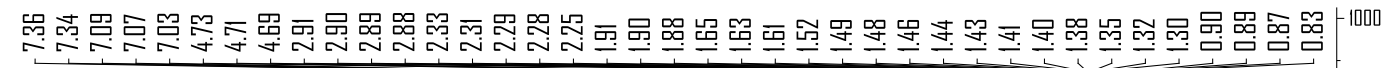

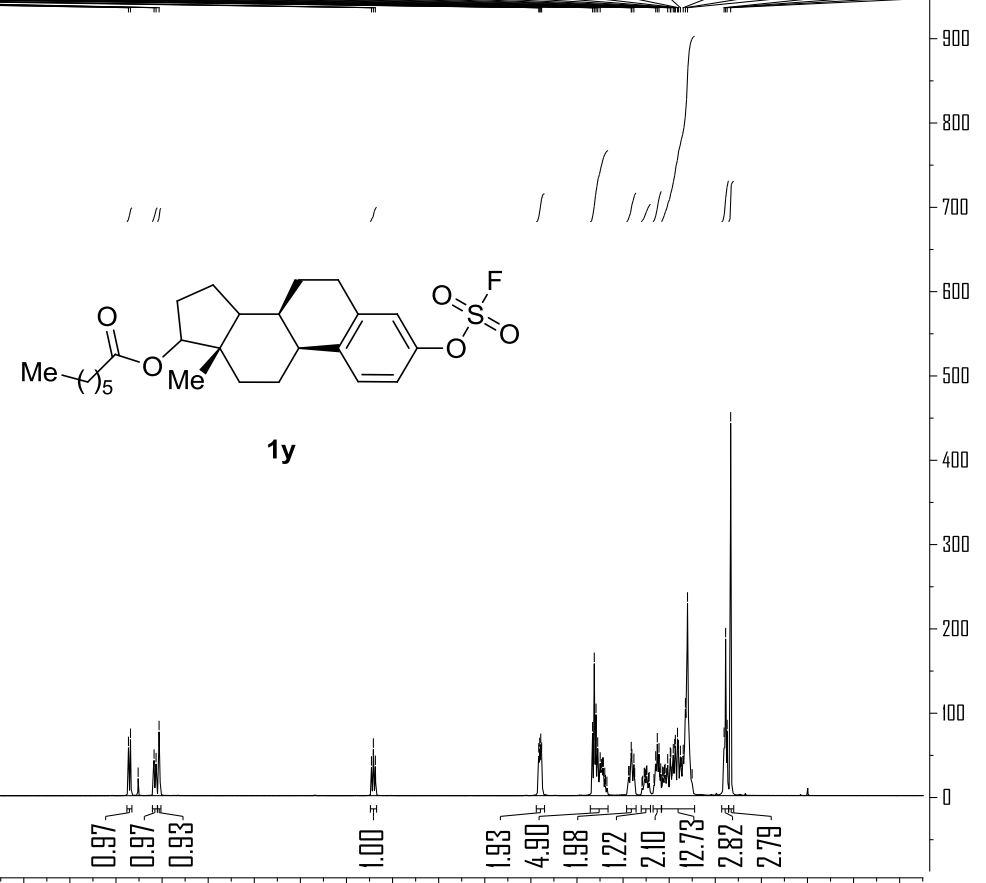

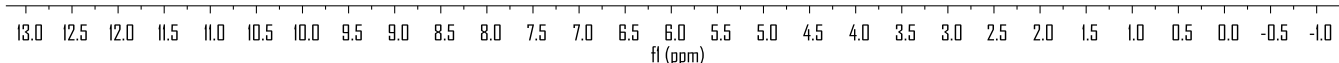



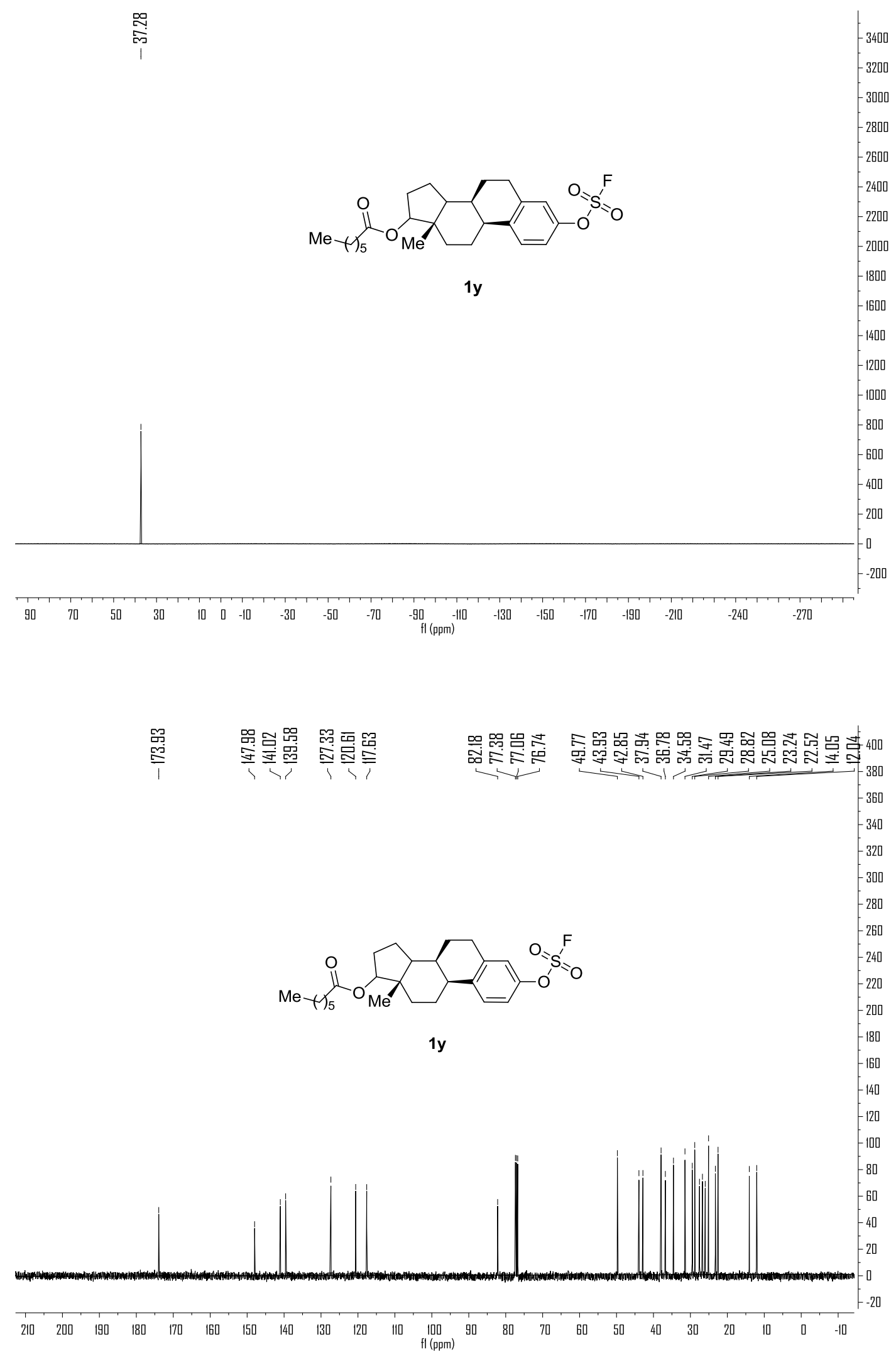

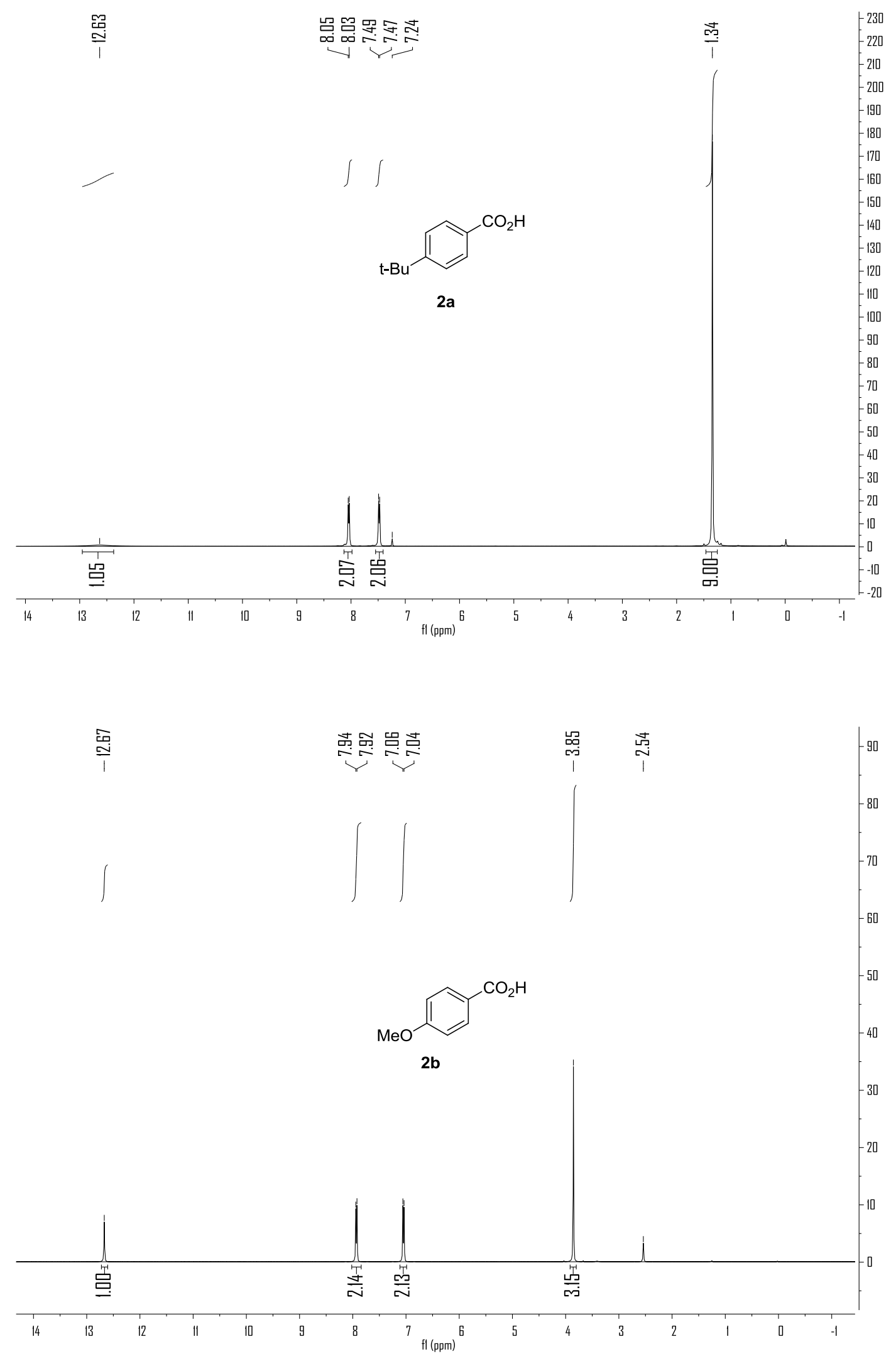


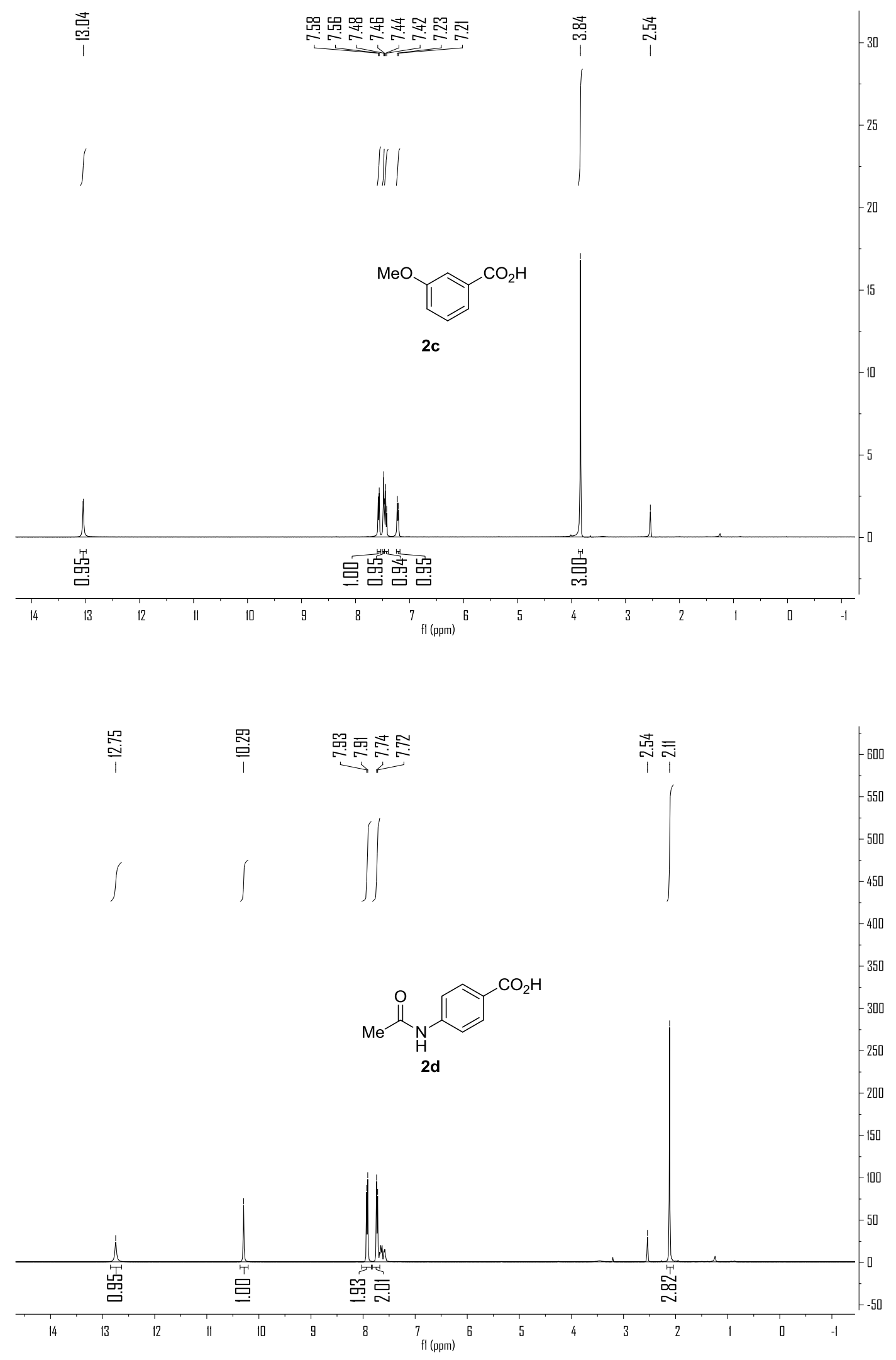



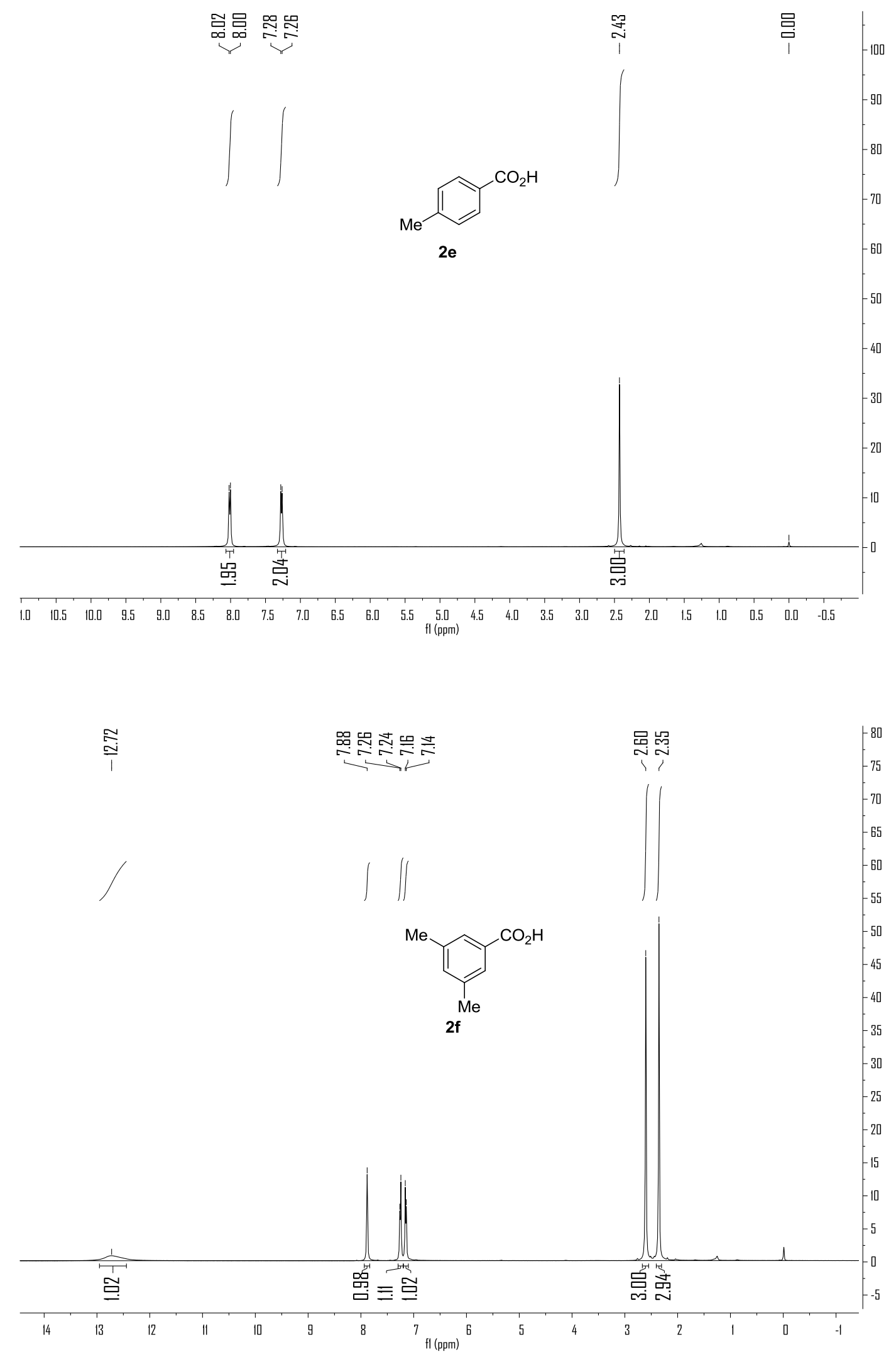

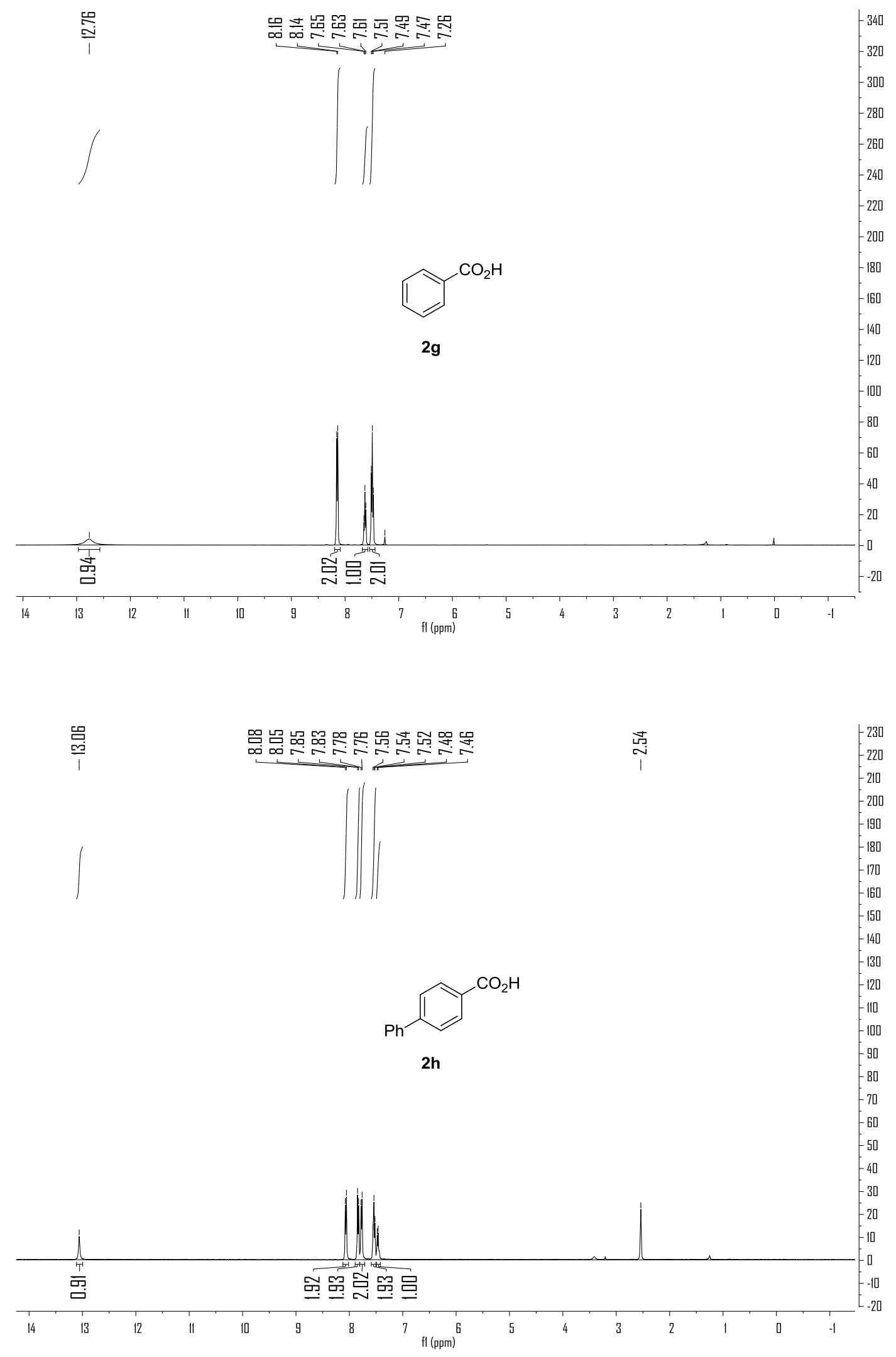


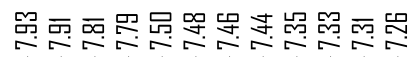
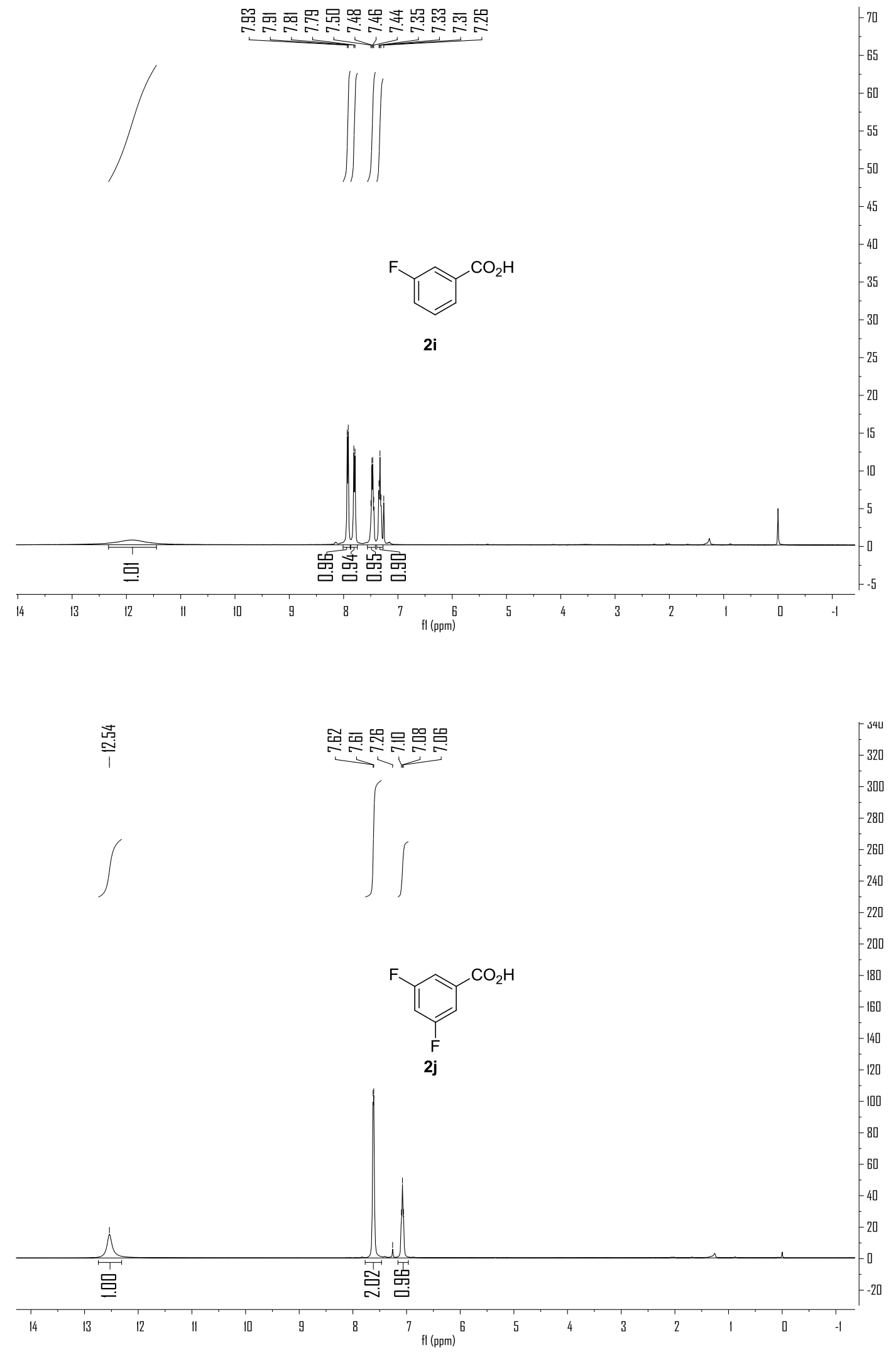

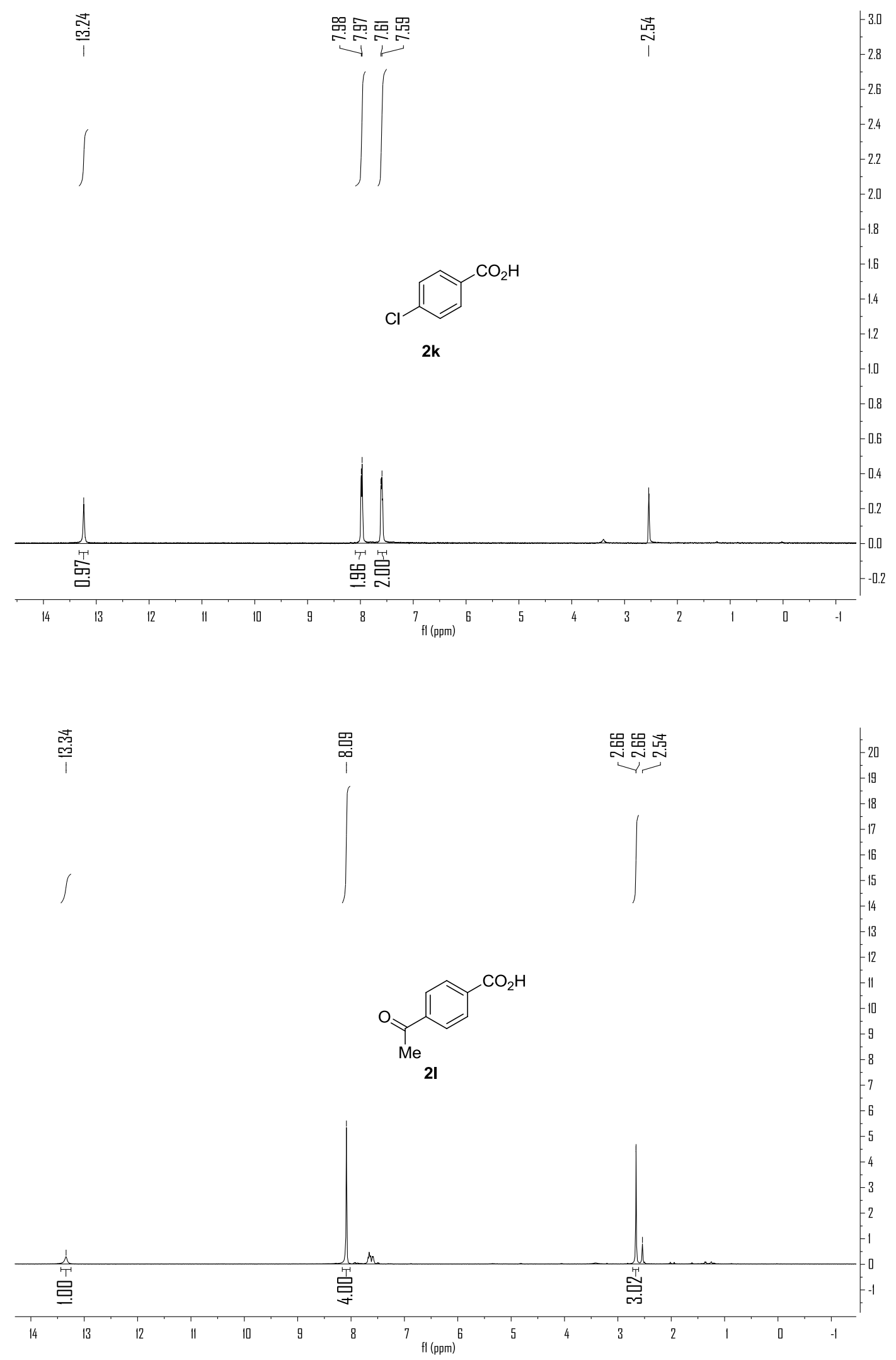

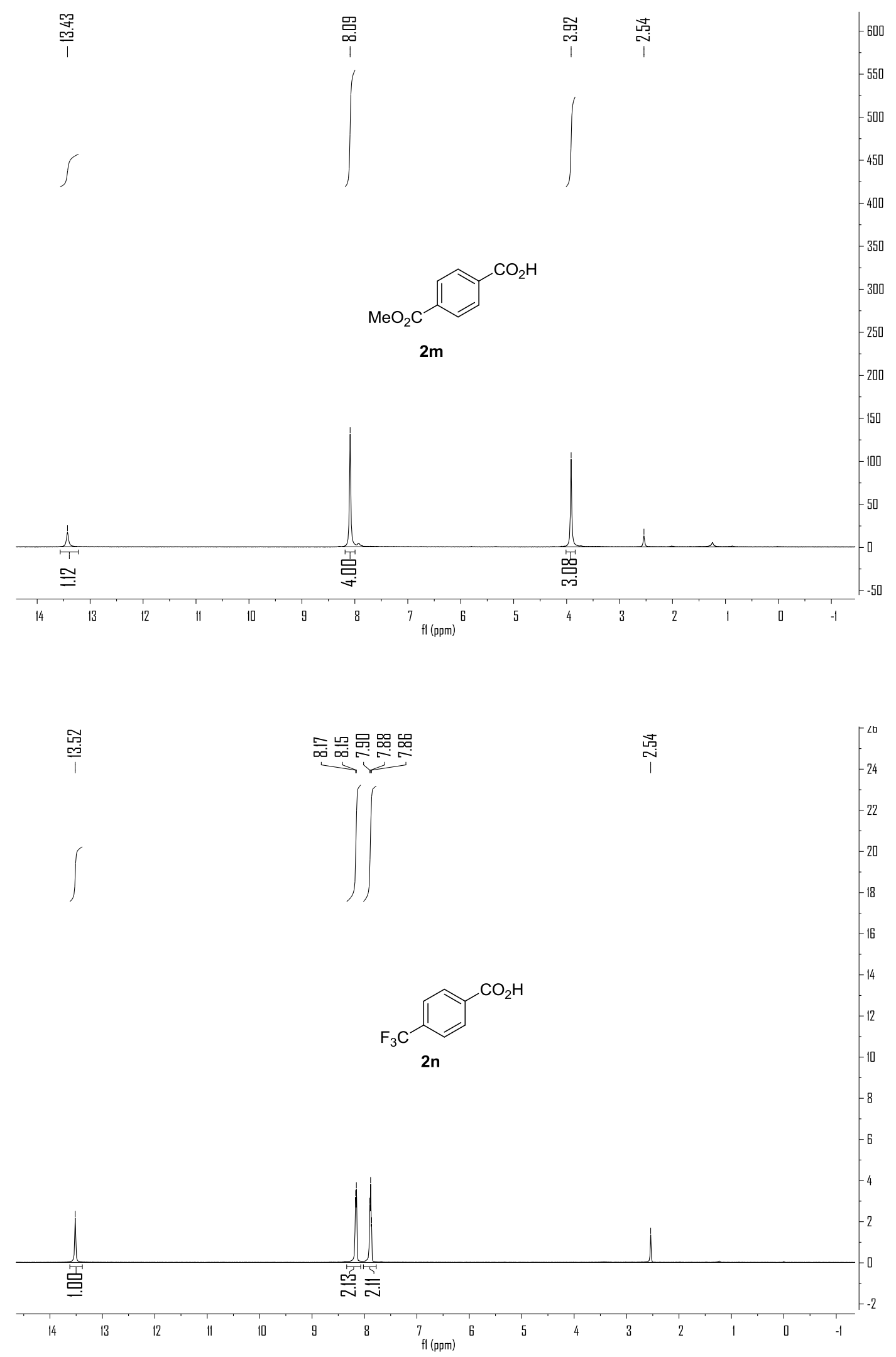

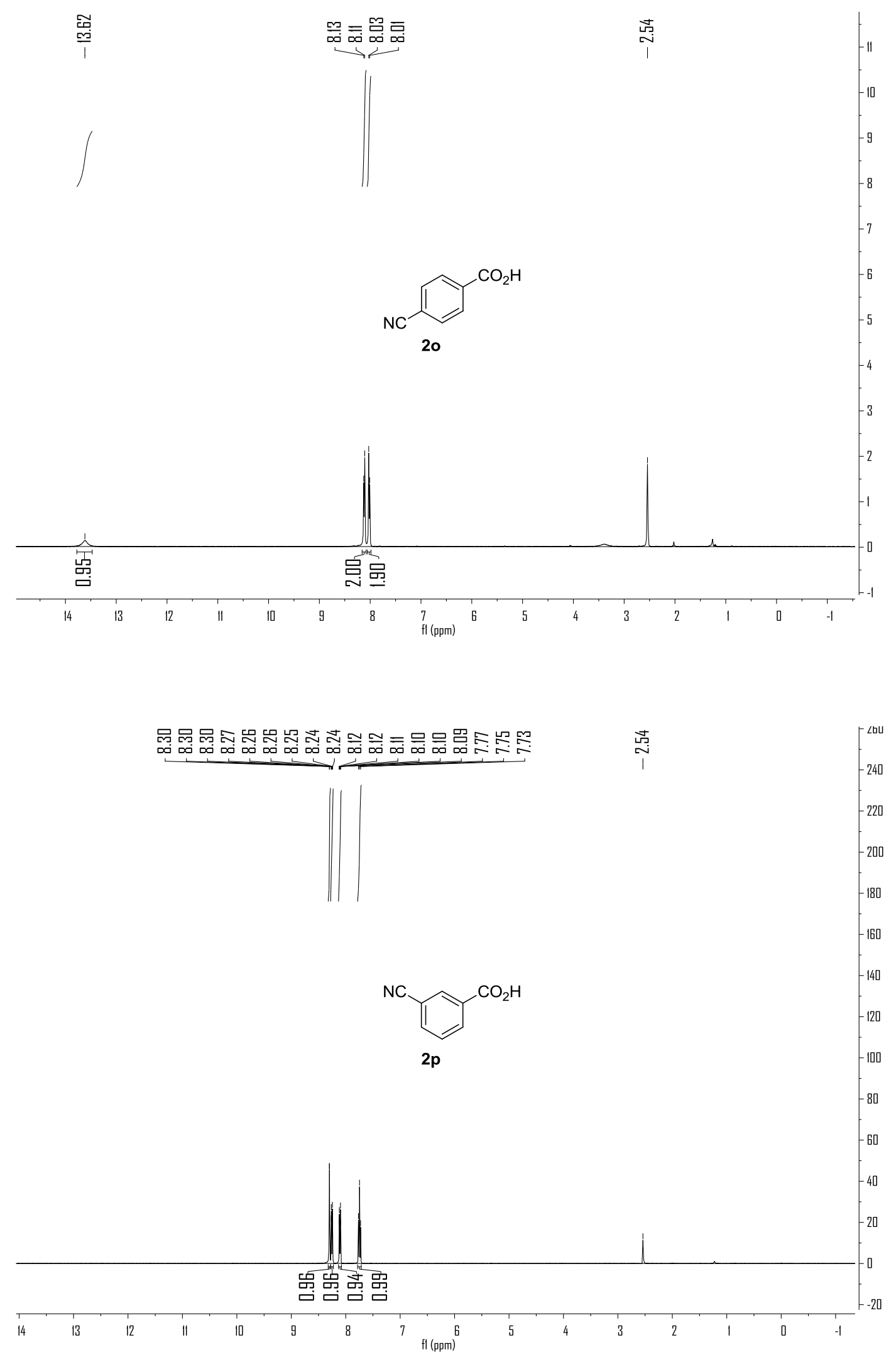

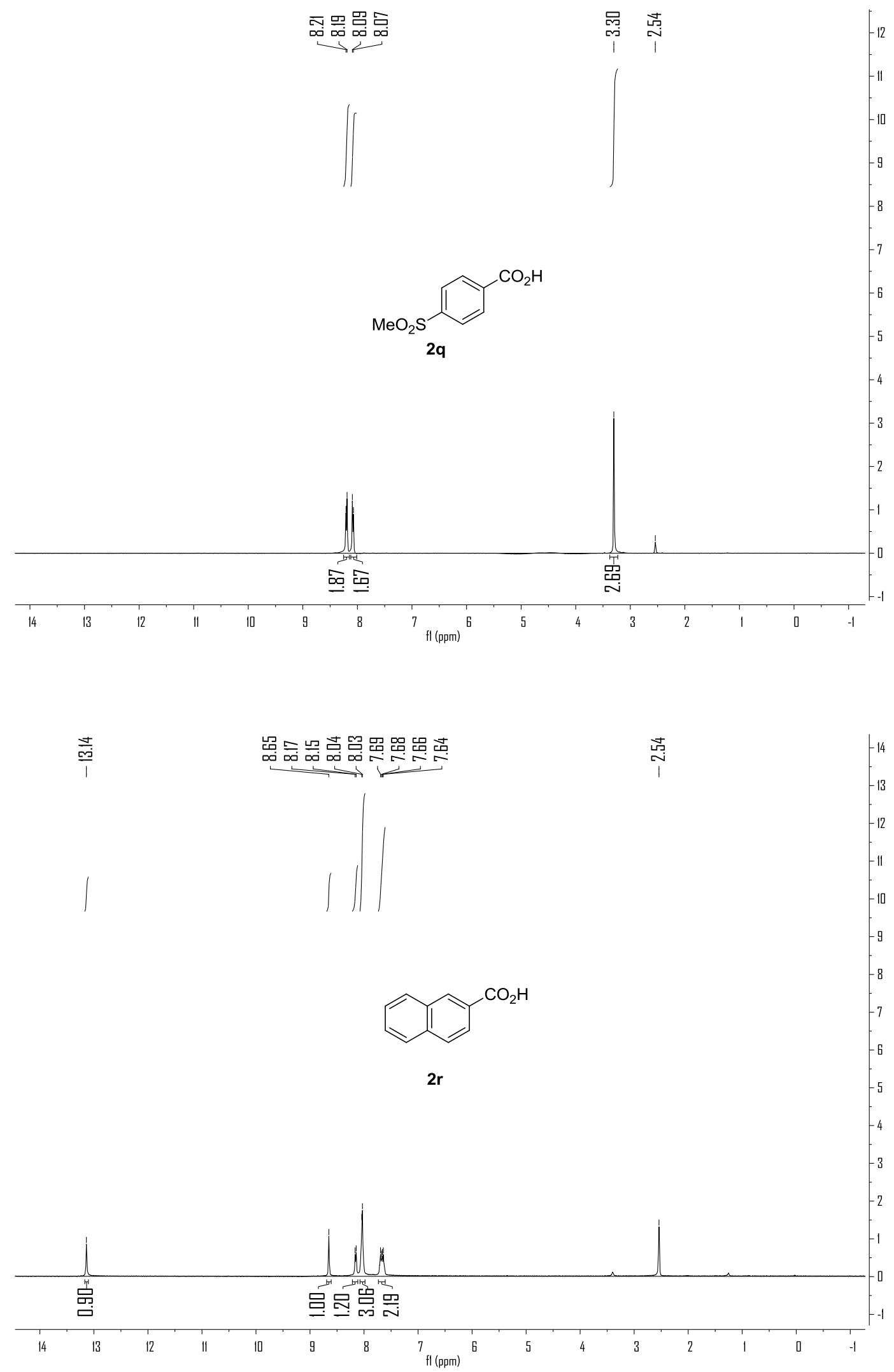

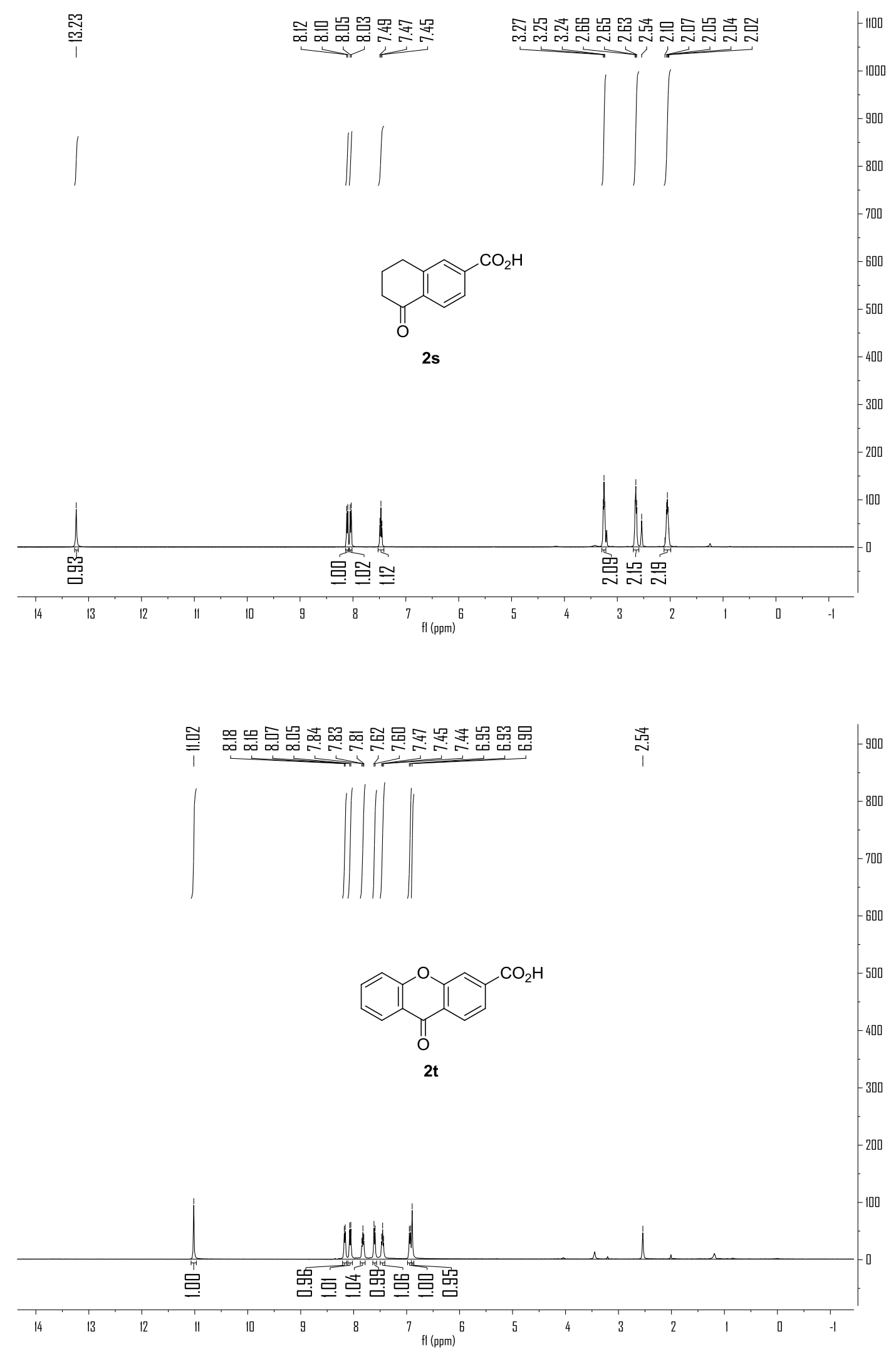

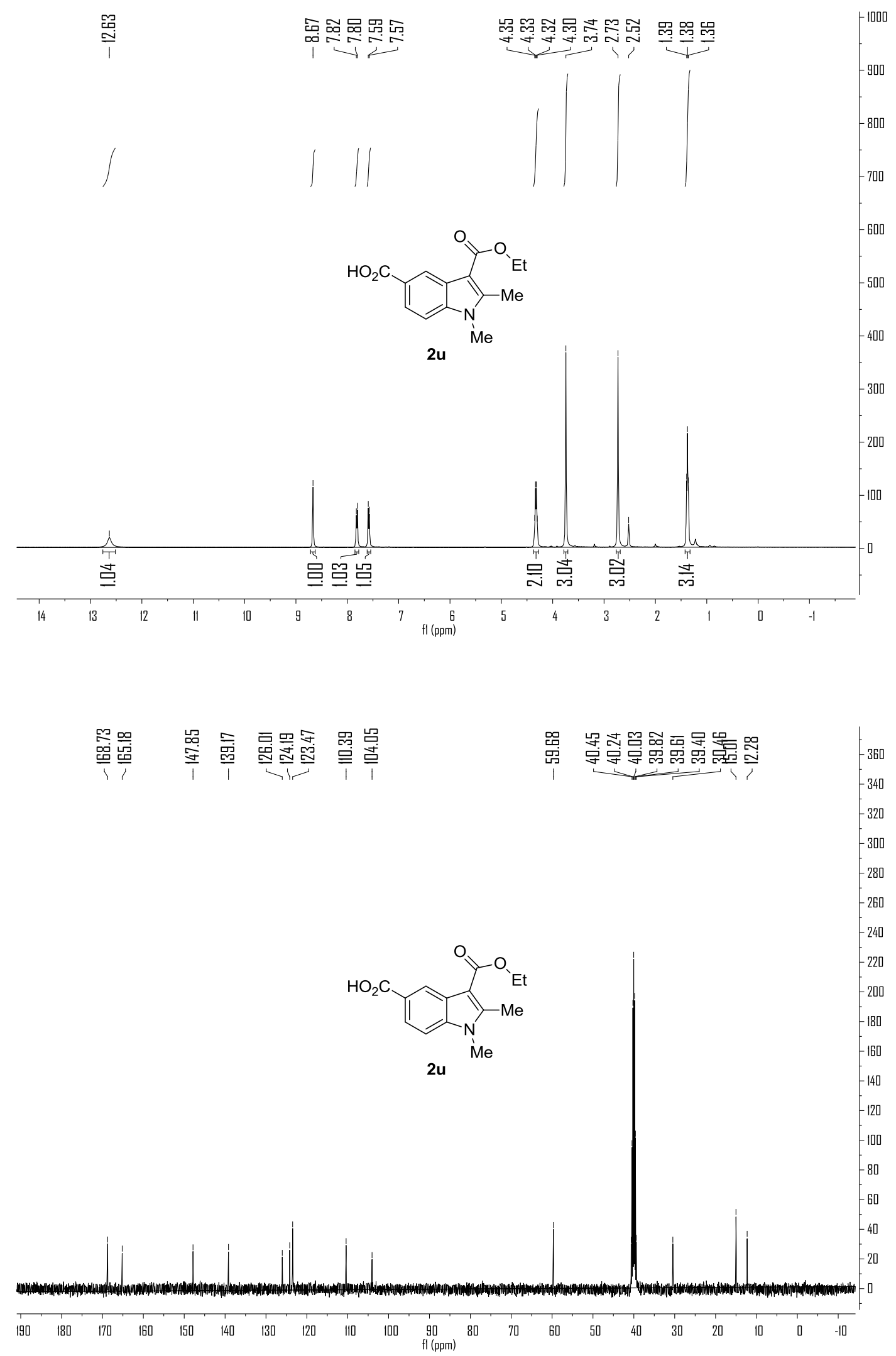

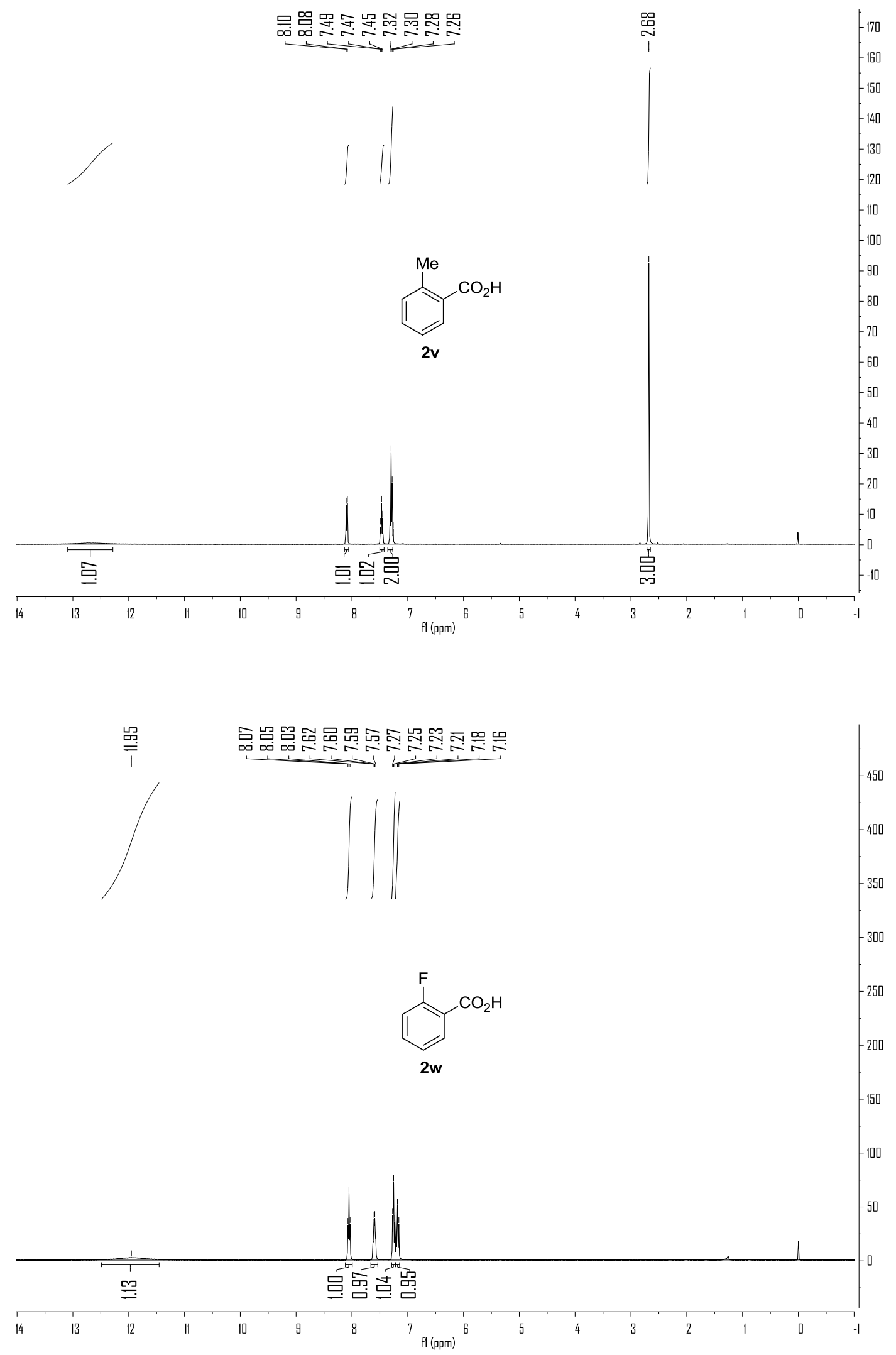


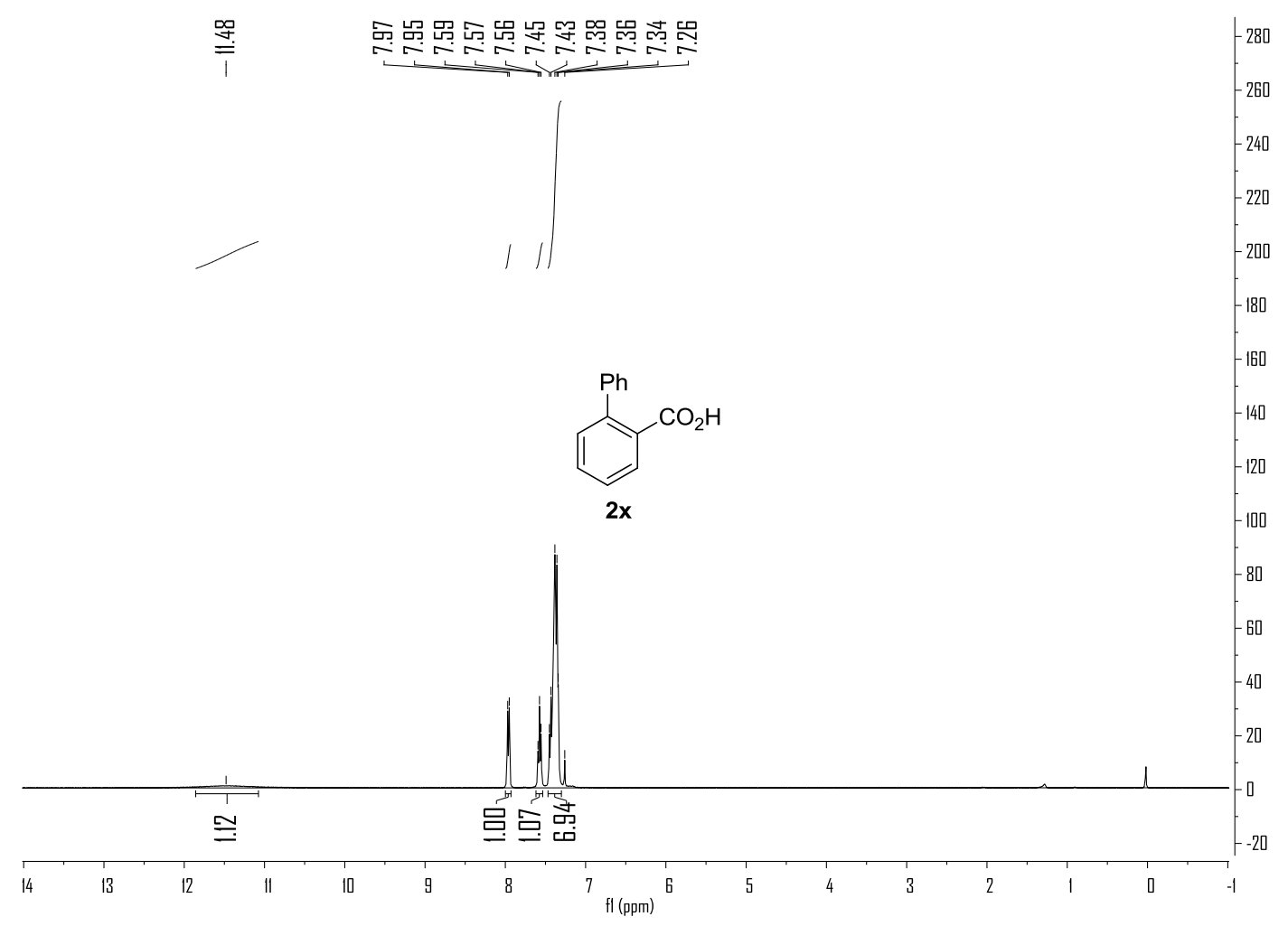

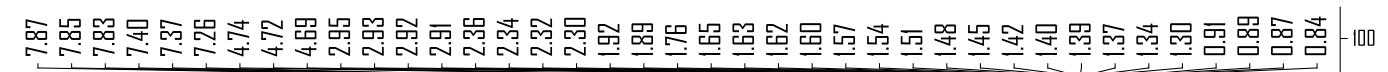

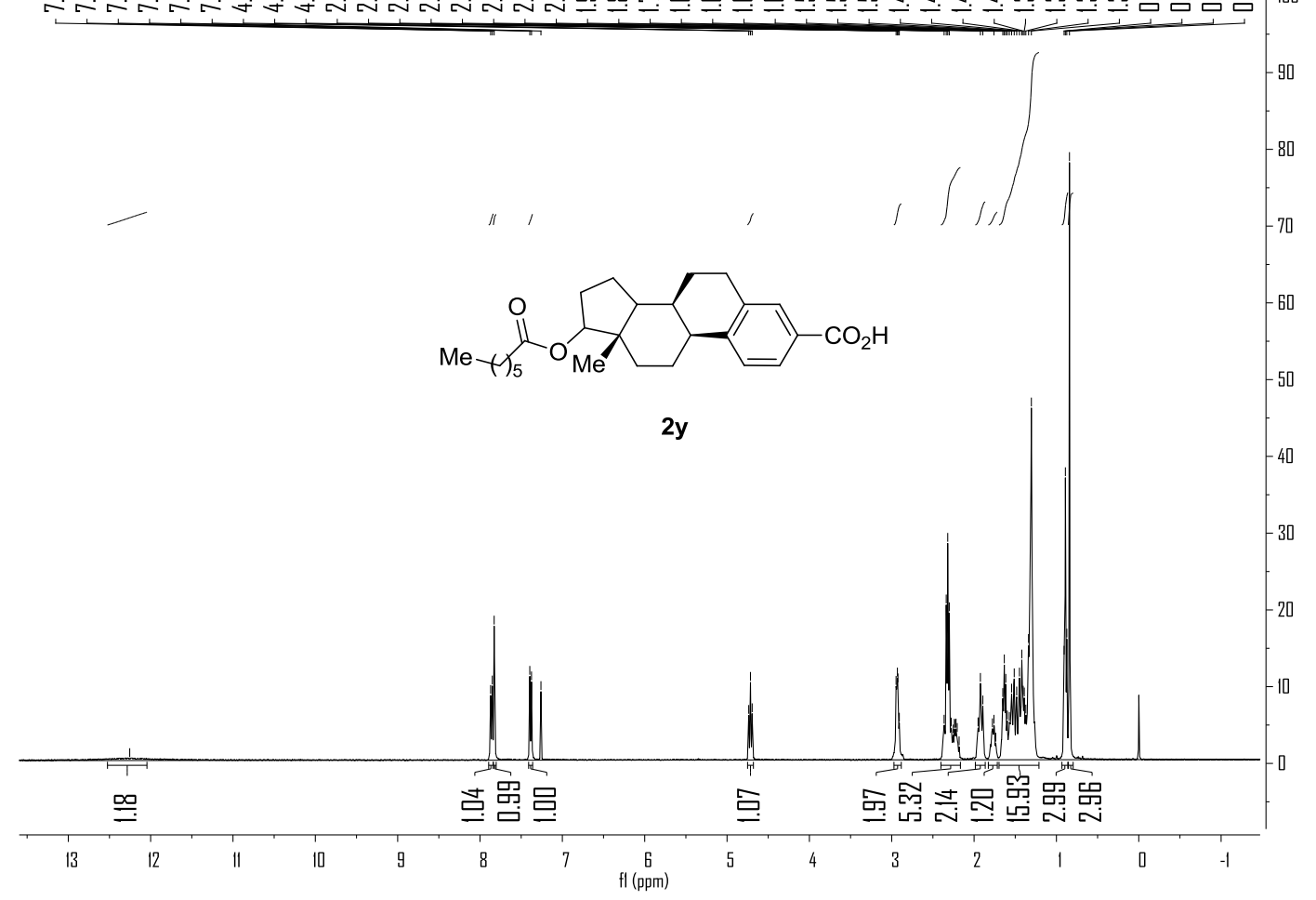



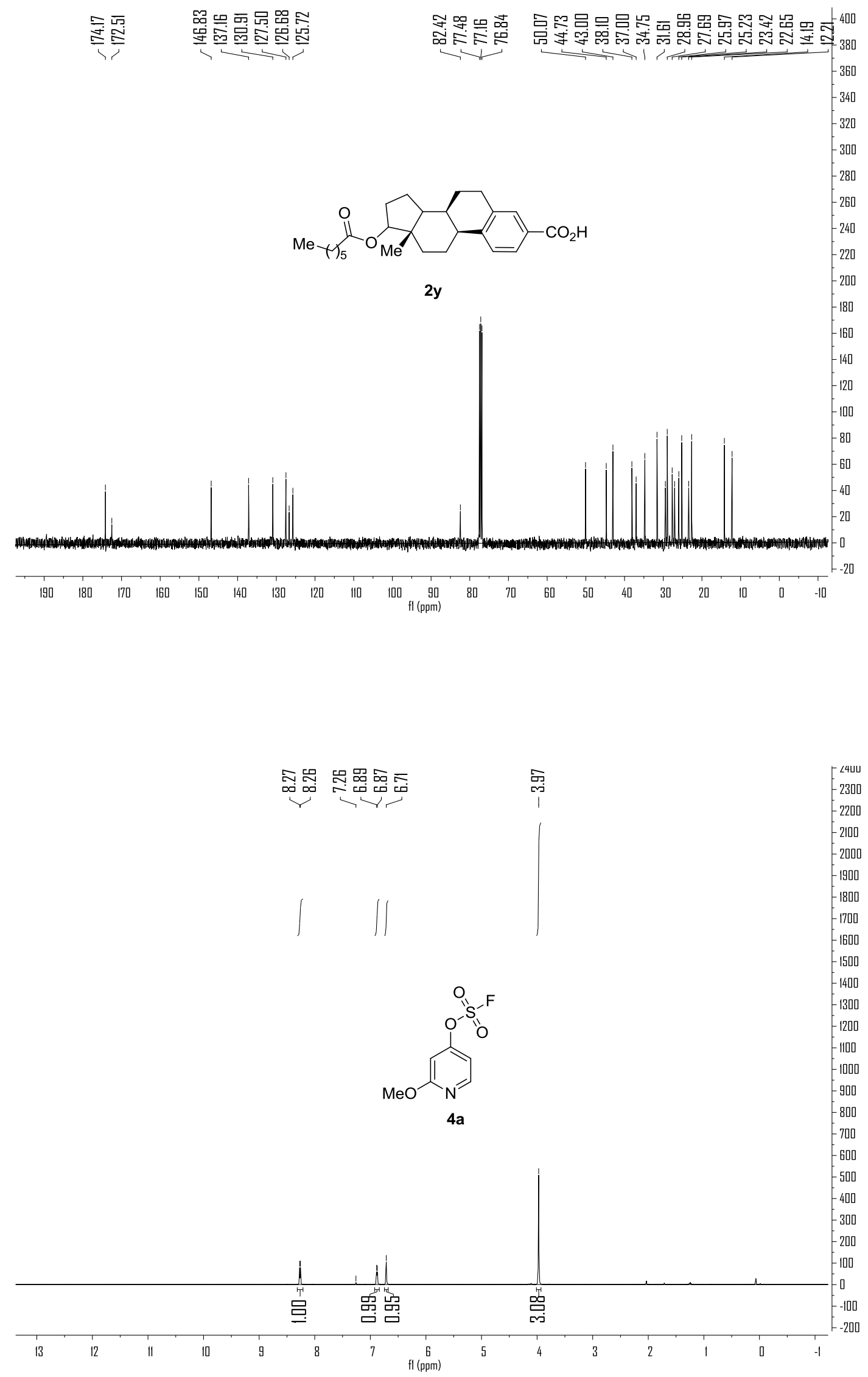

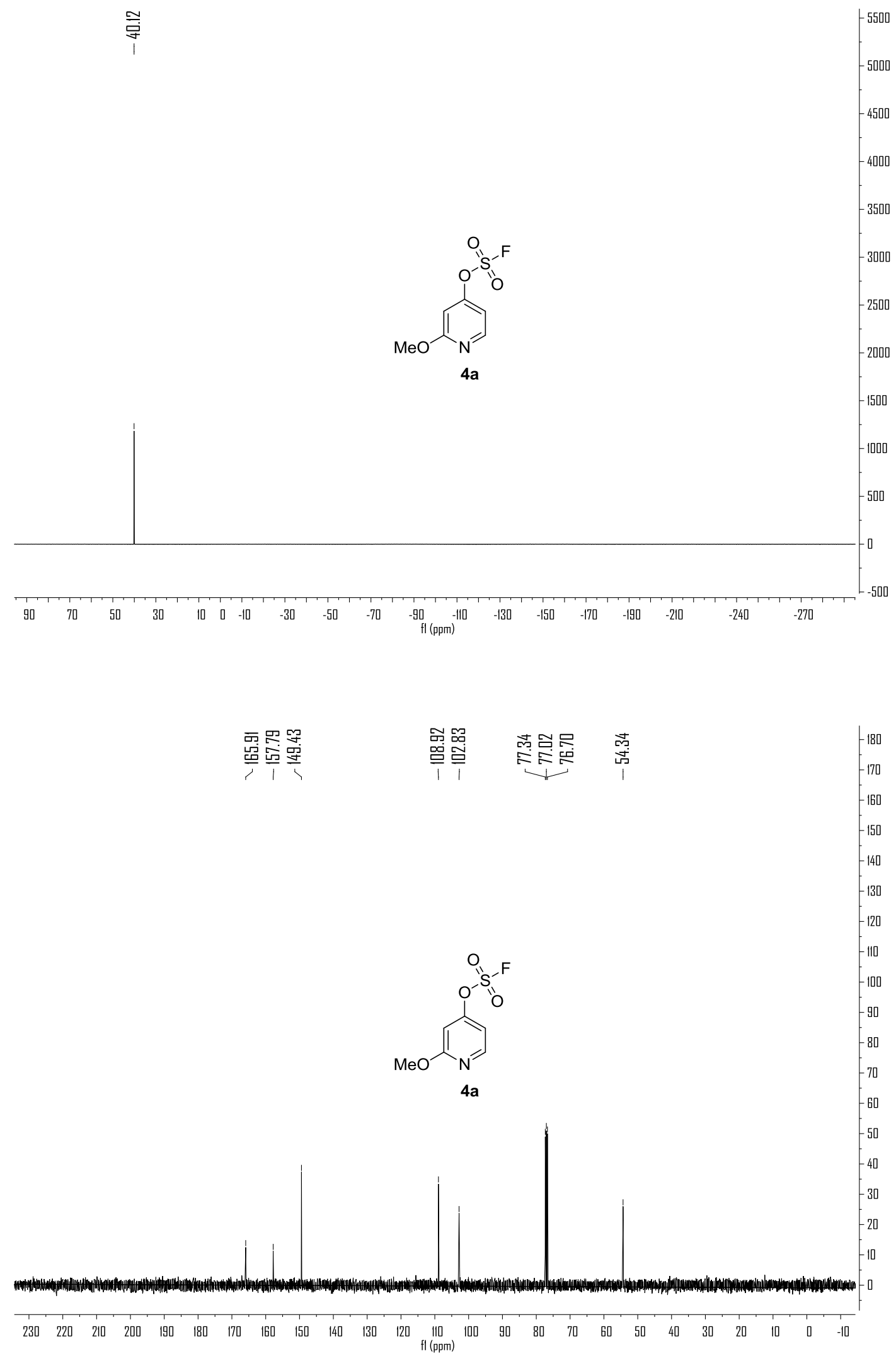

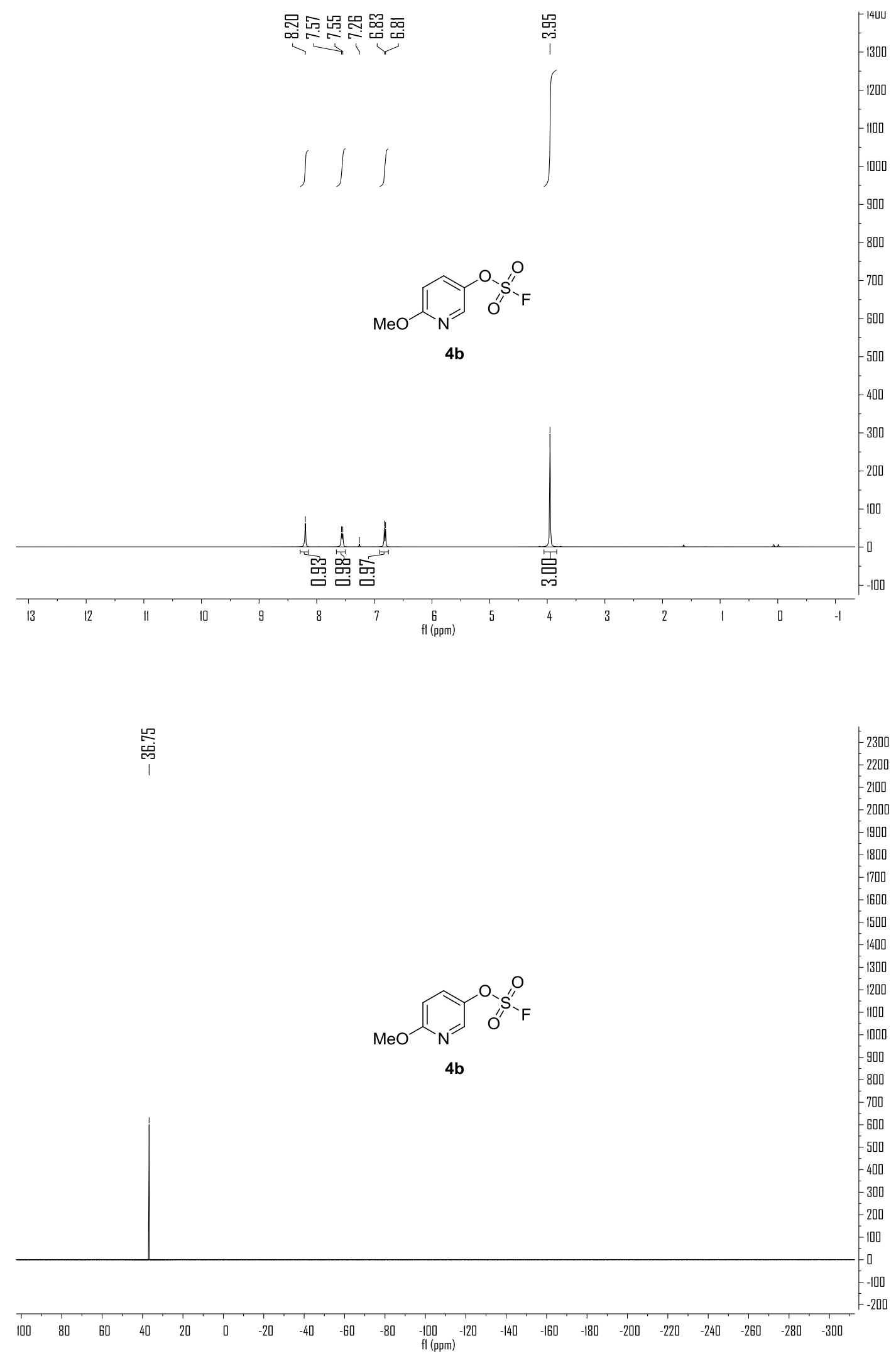

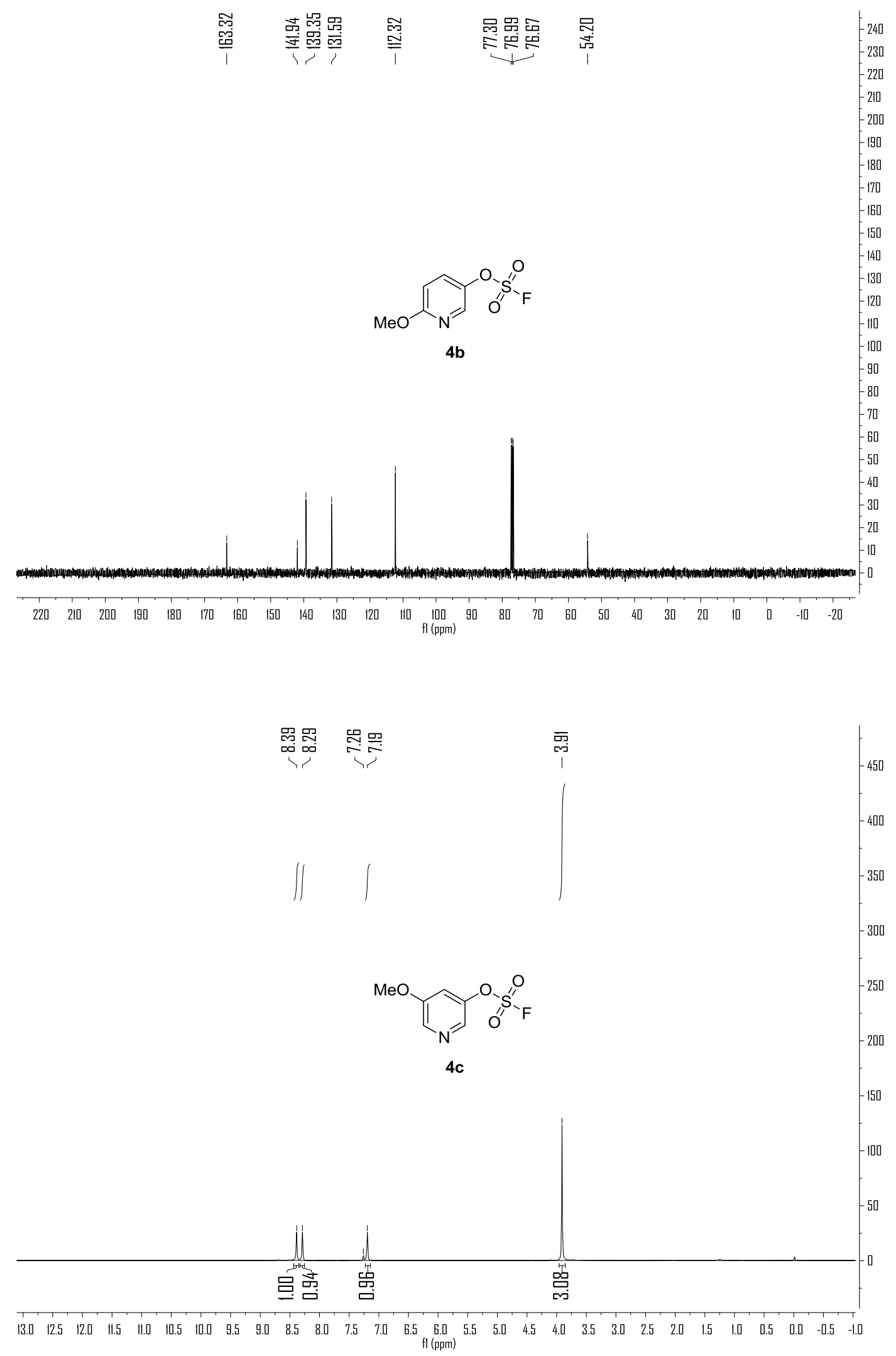


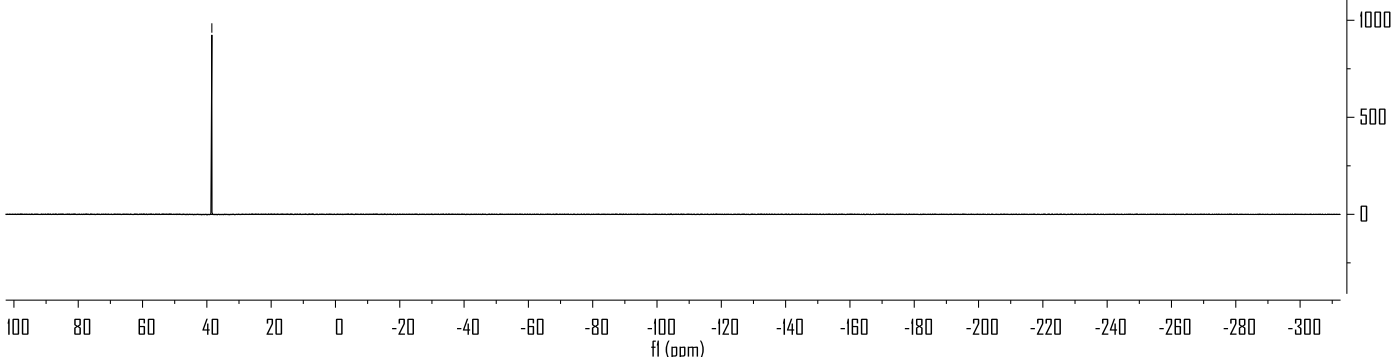

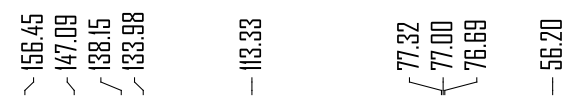

300

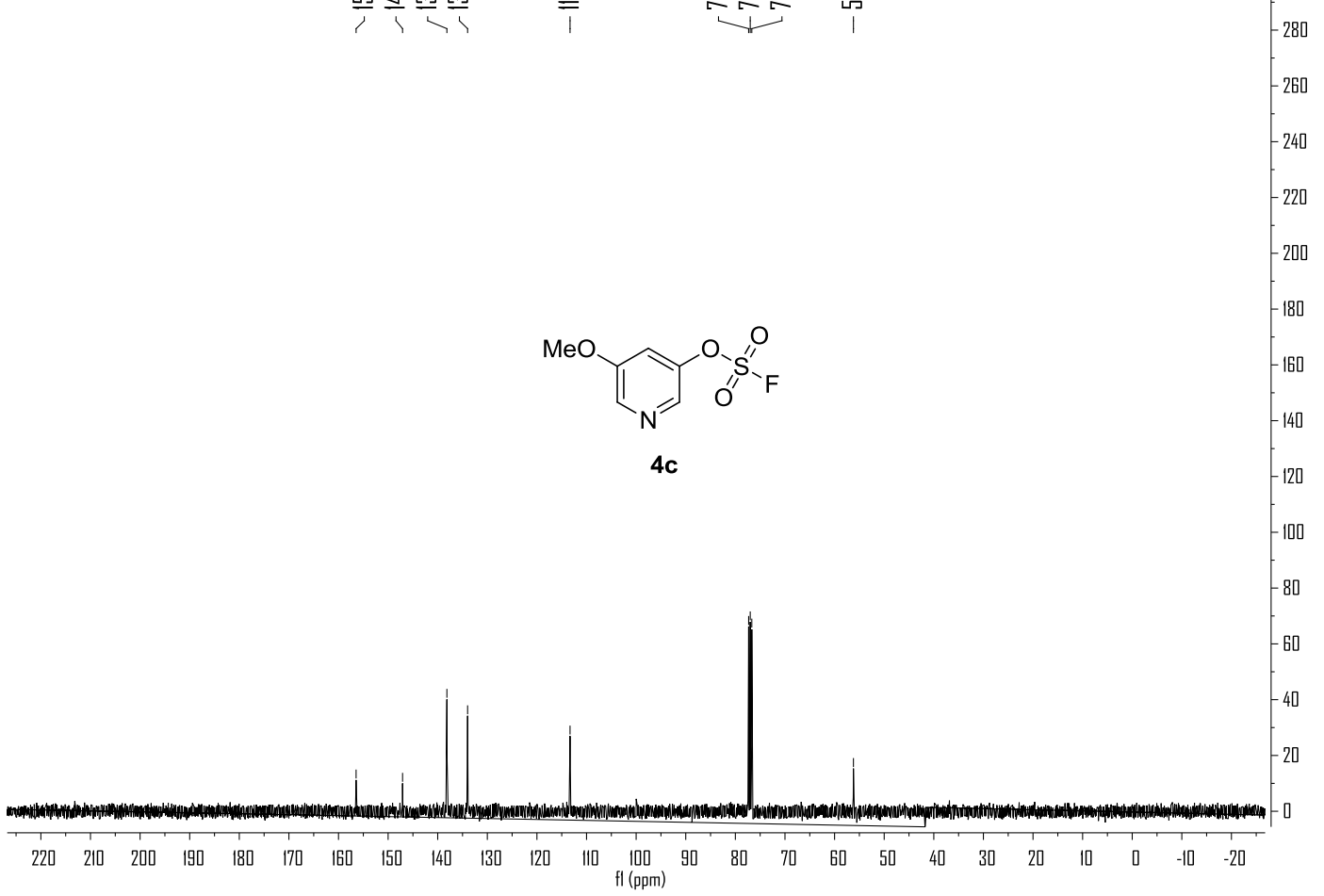



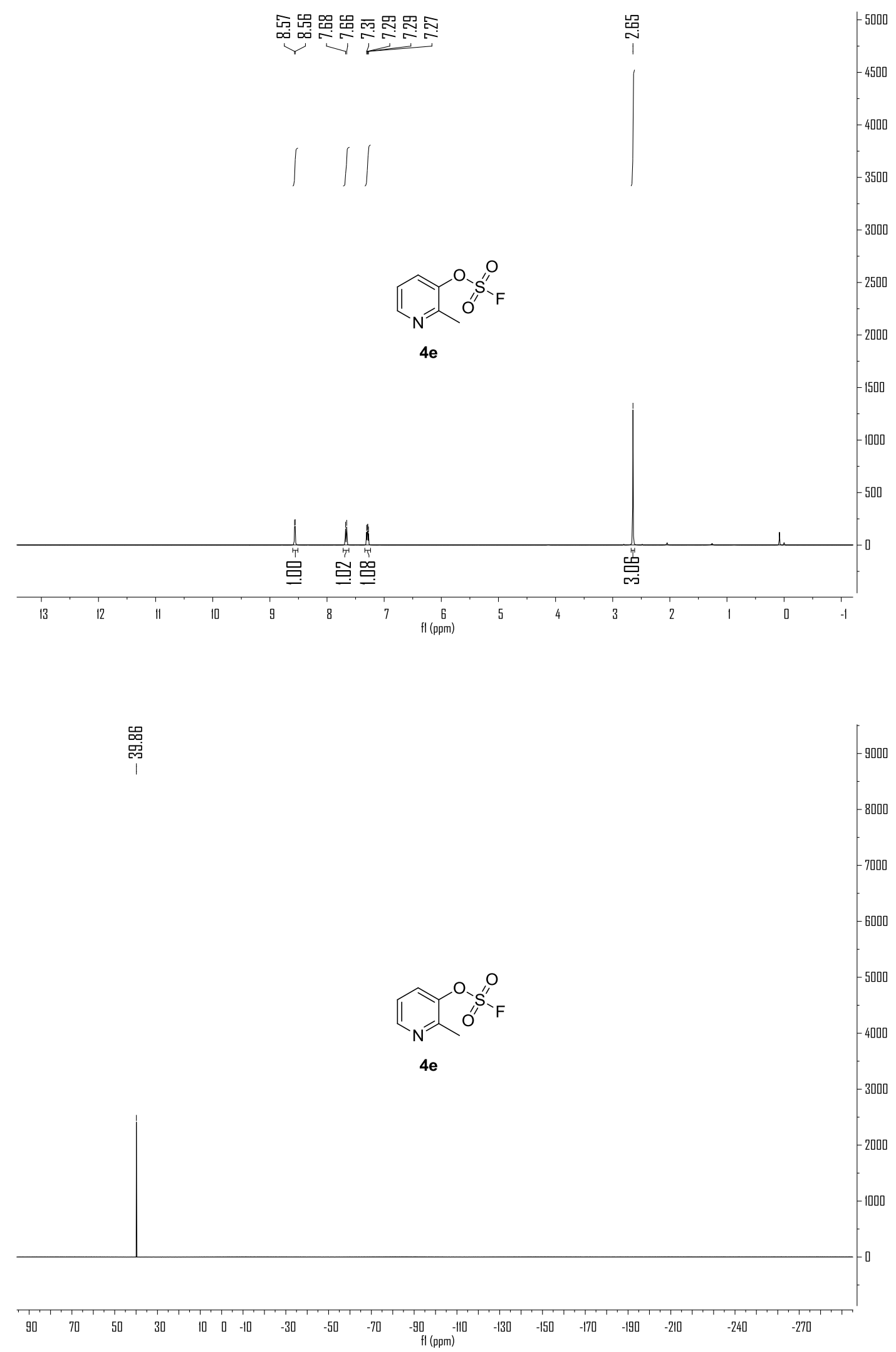

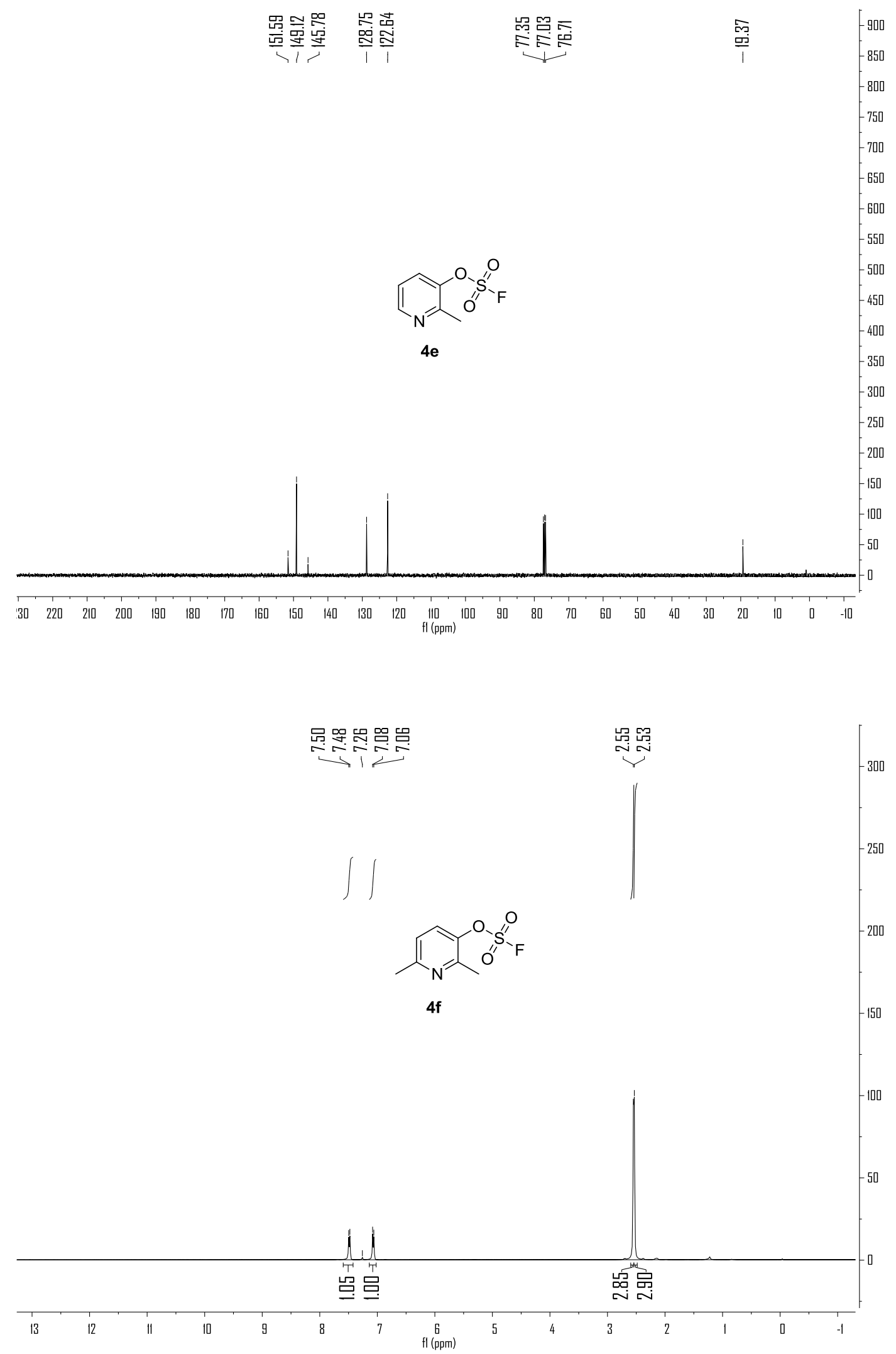

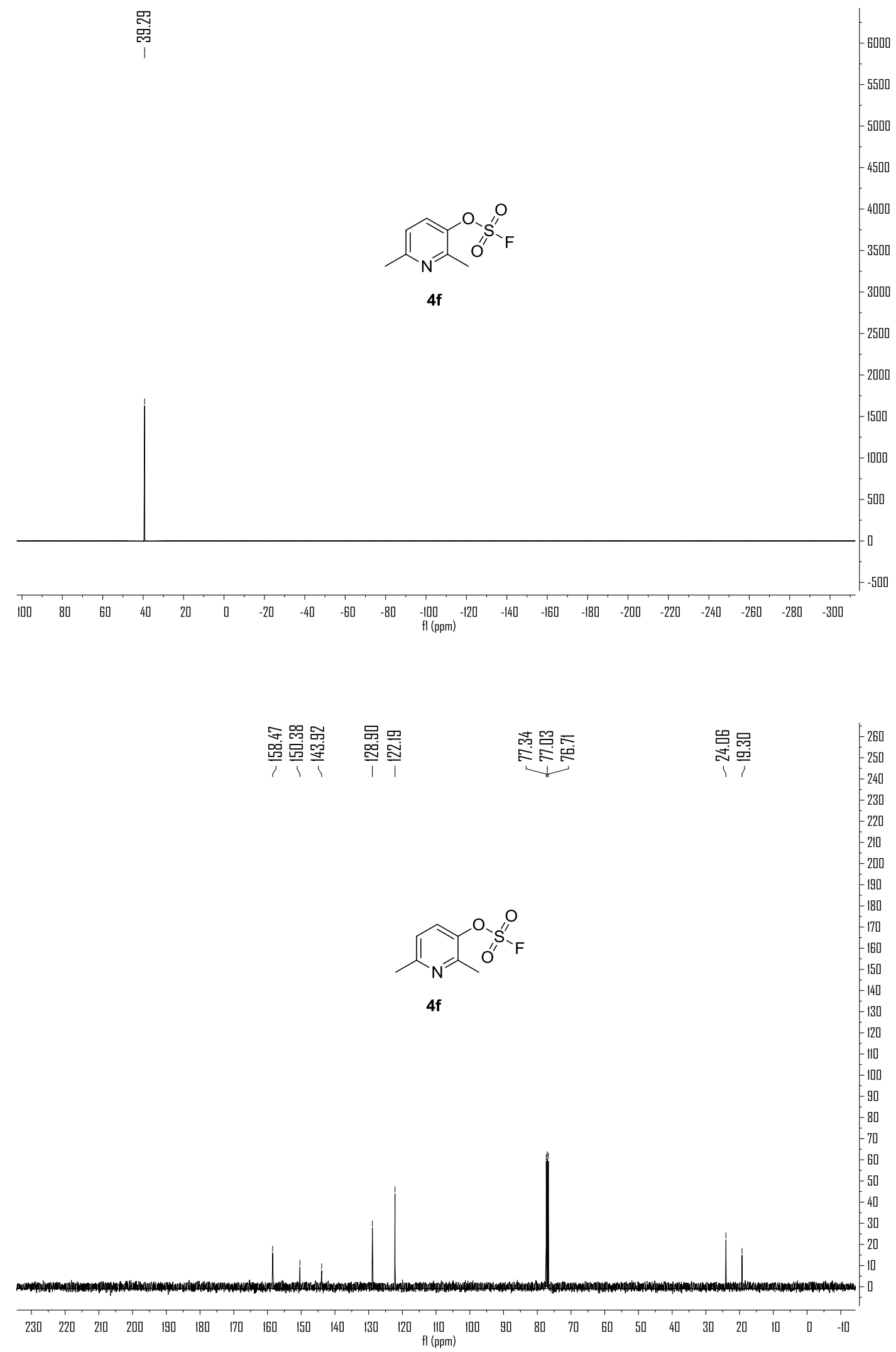

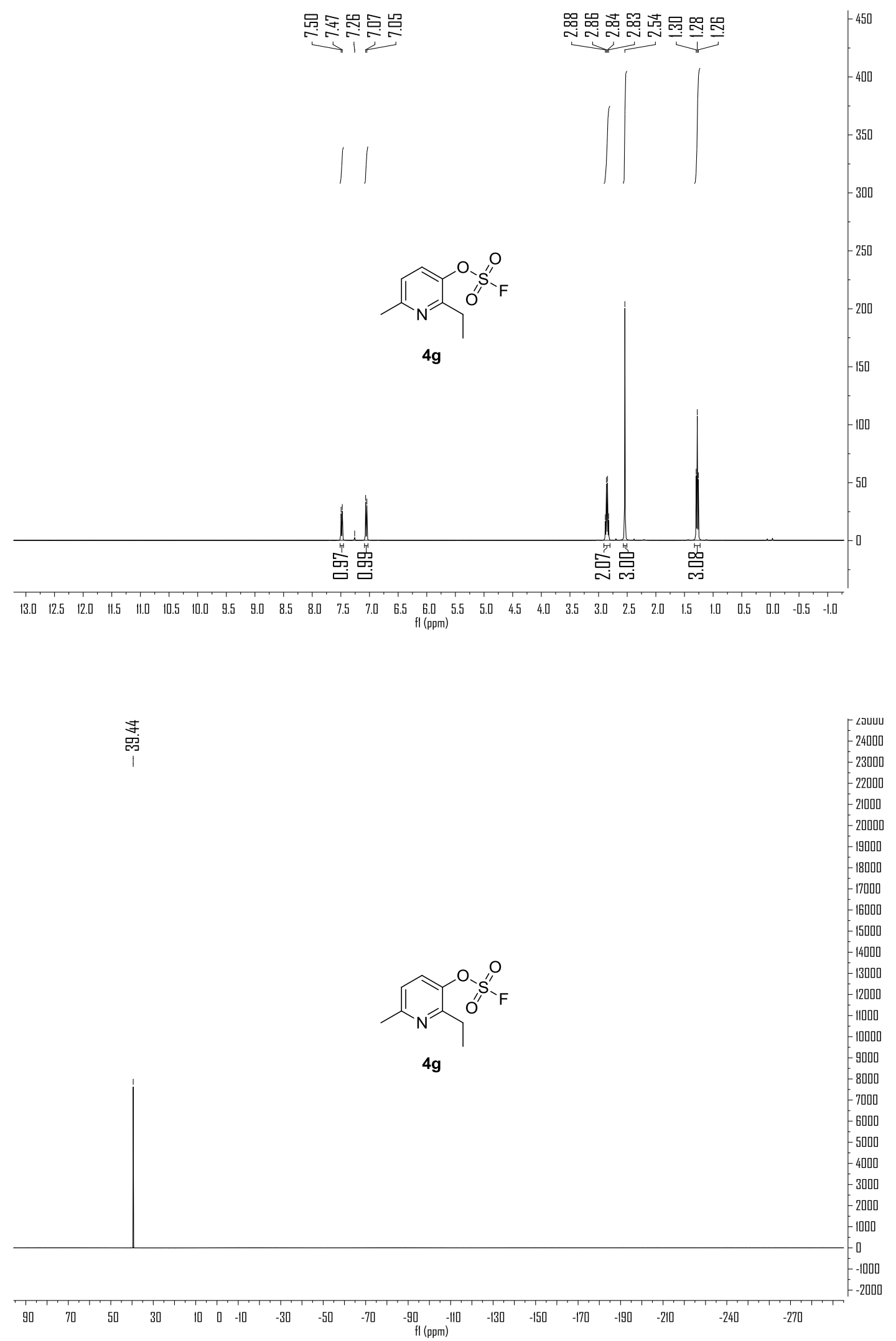

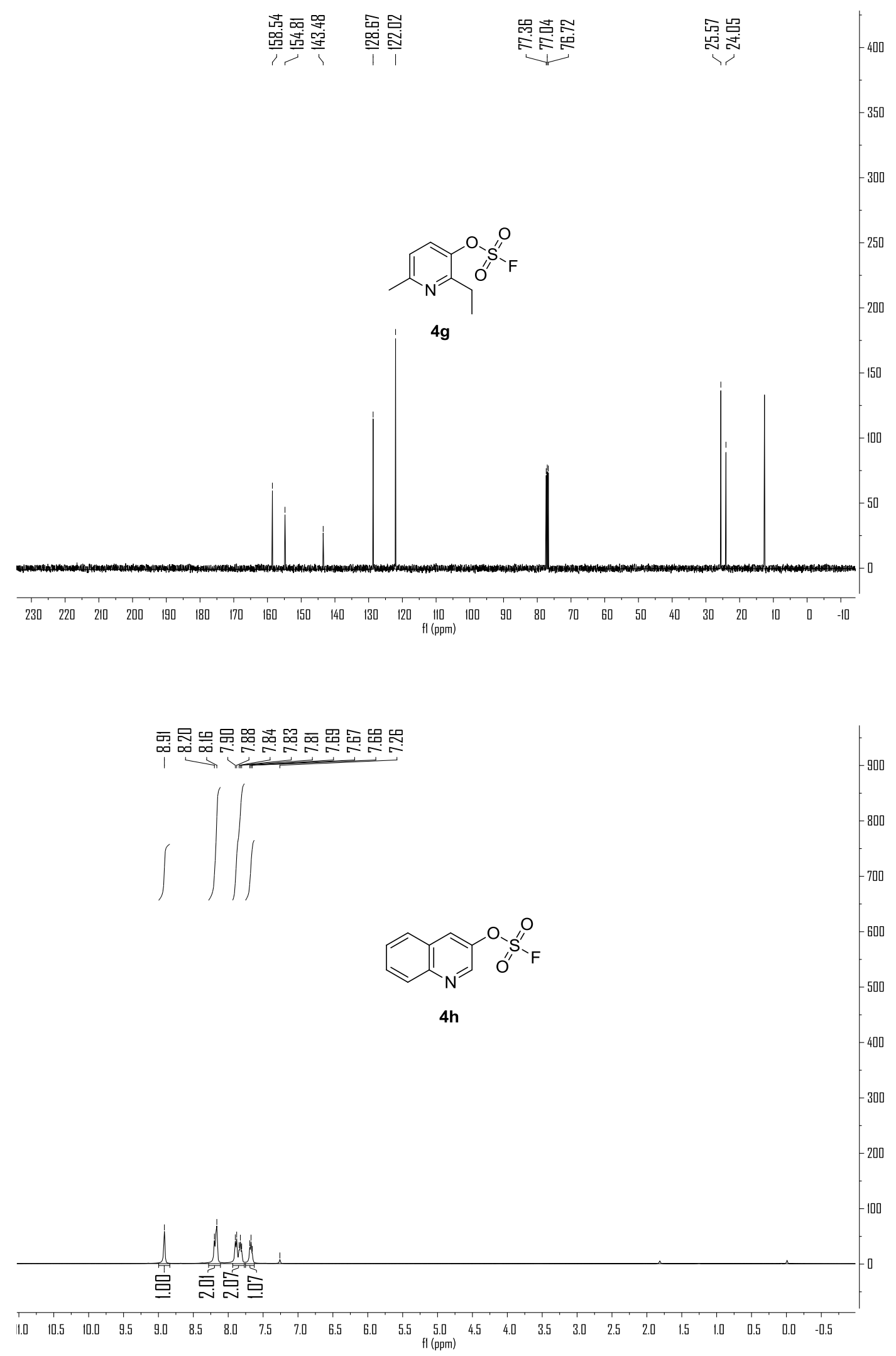


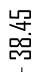
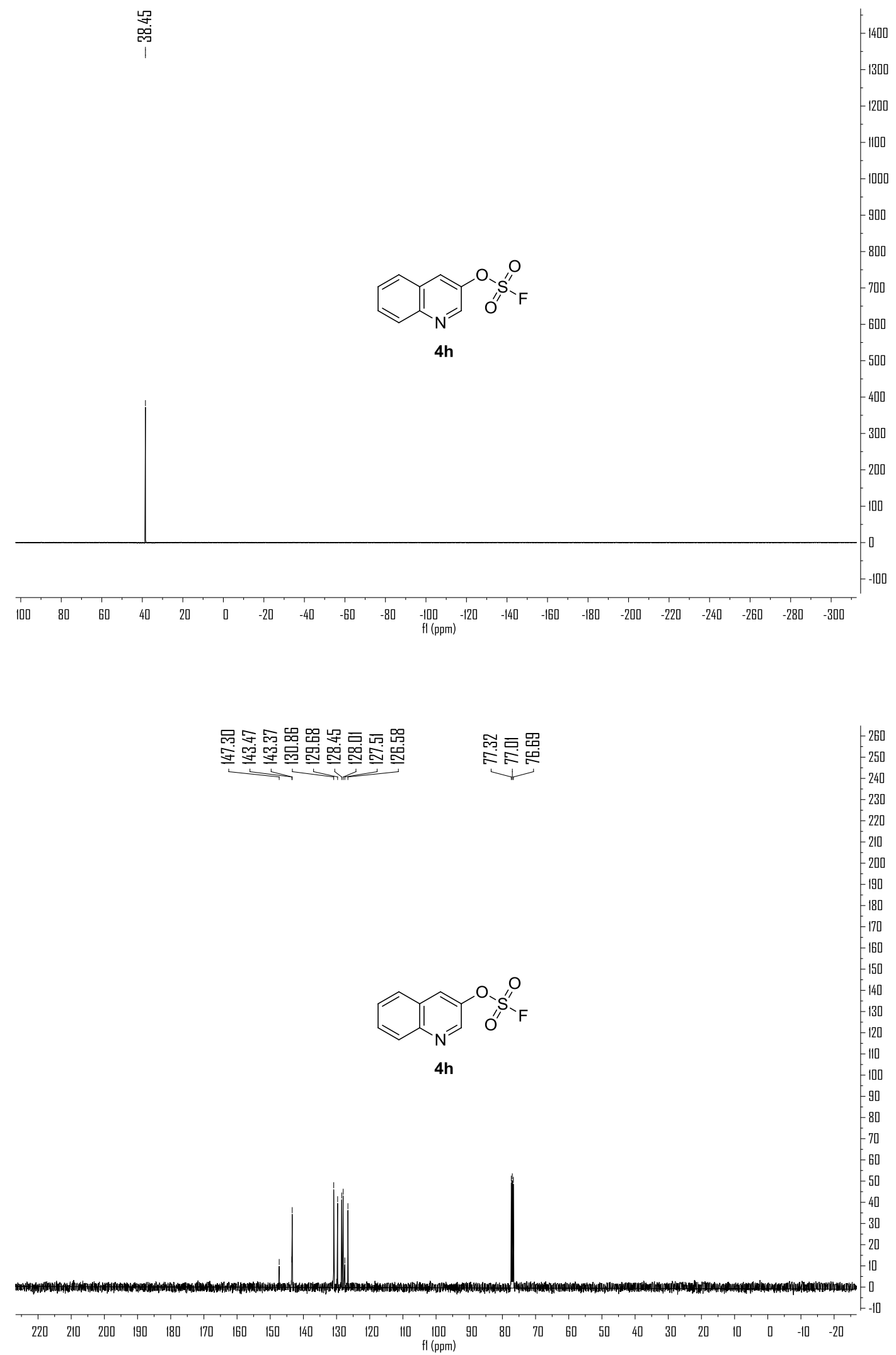

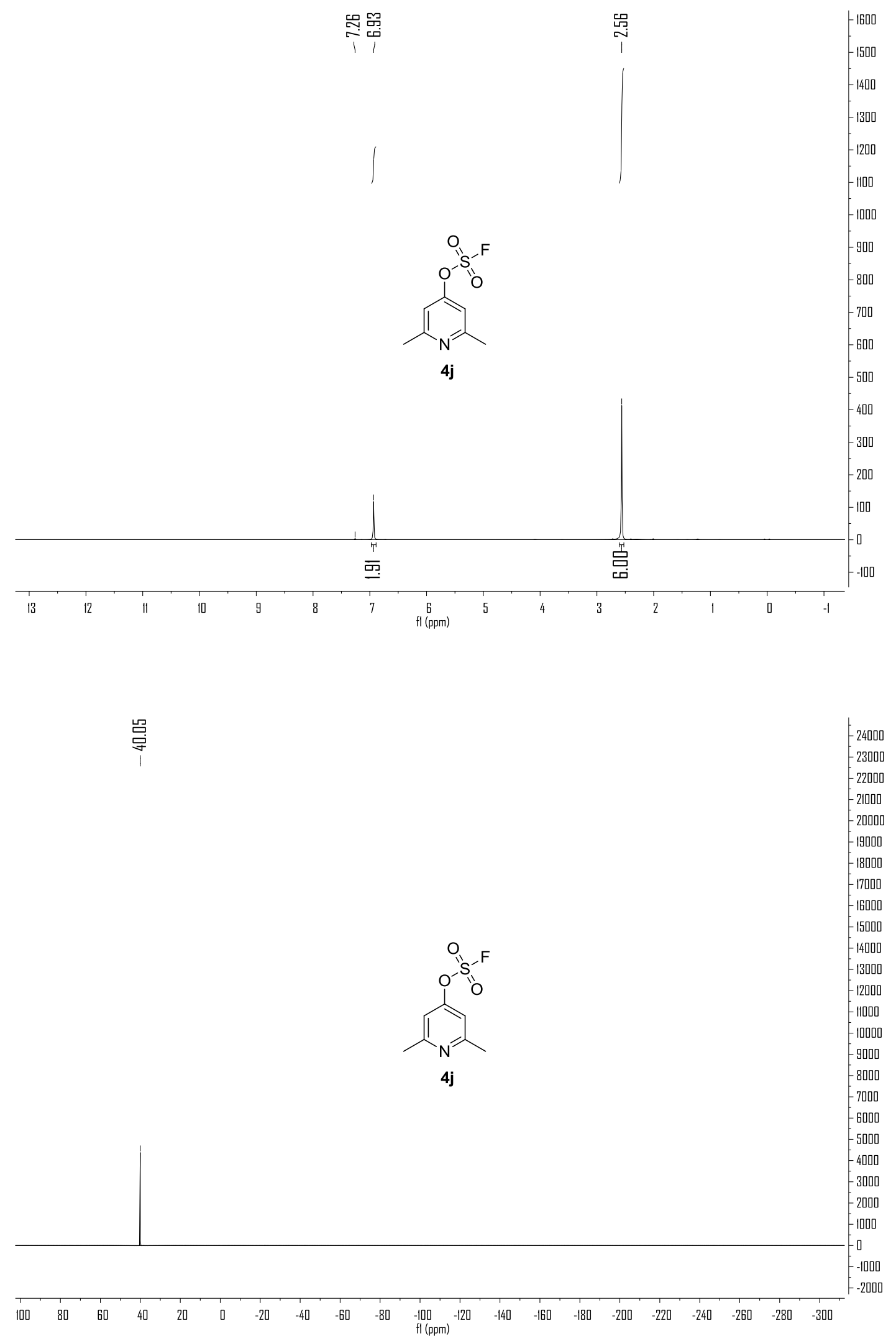

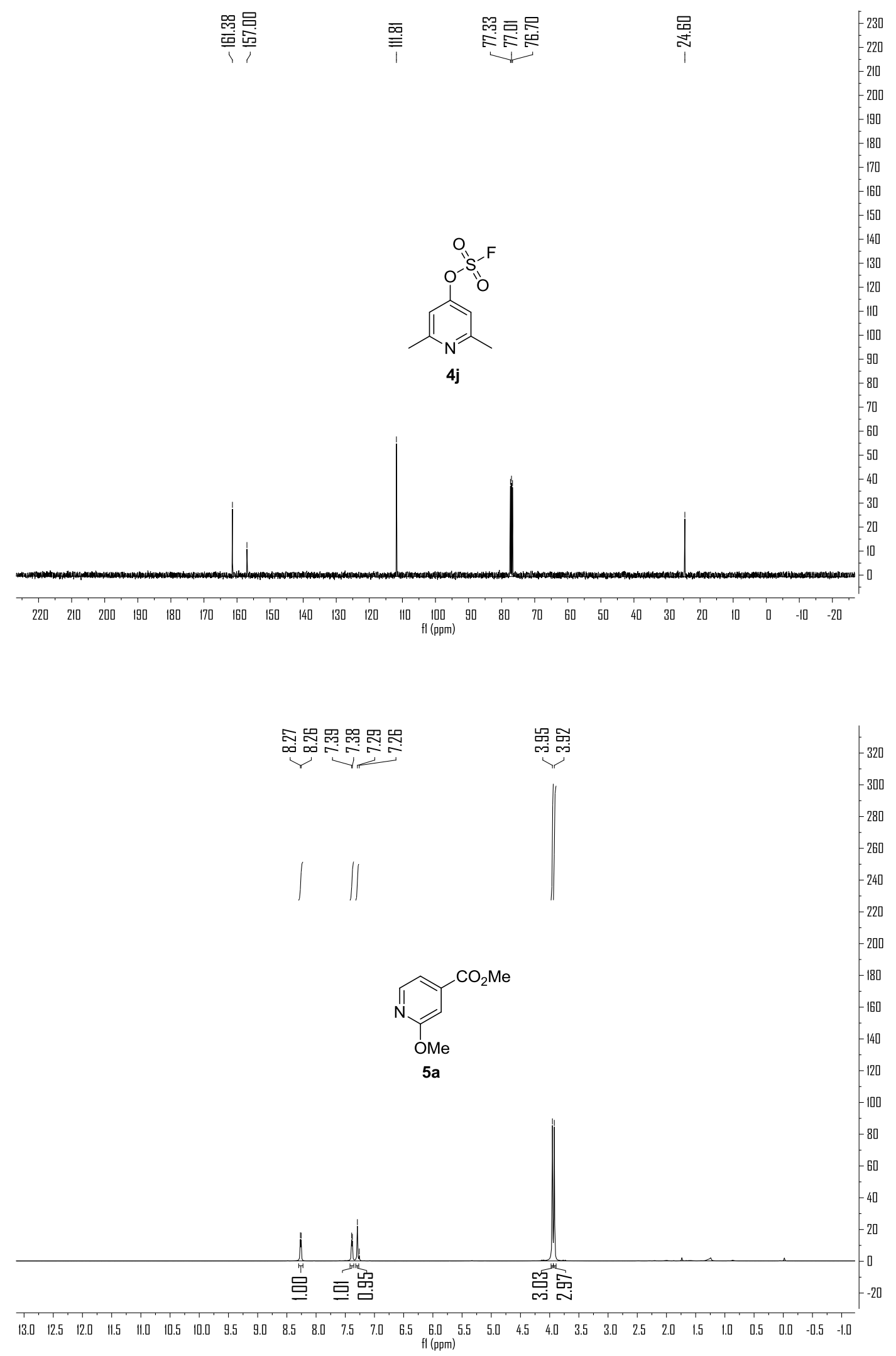

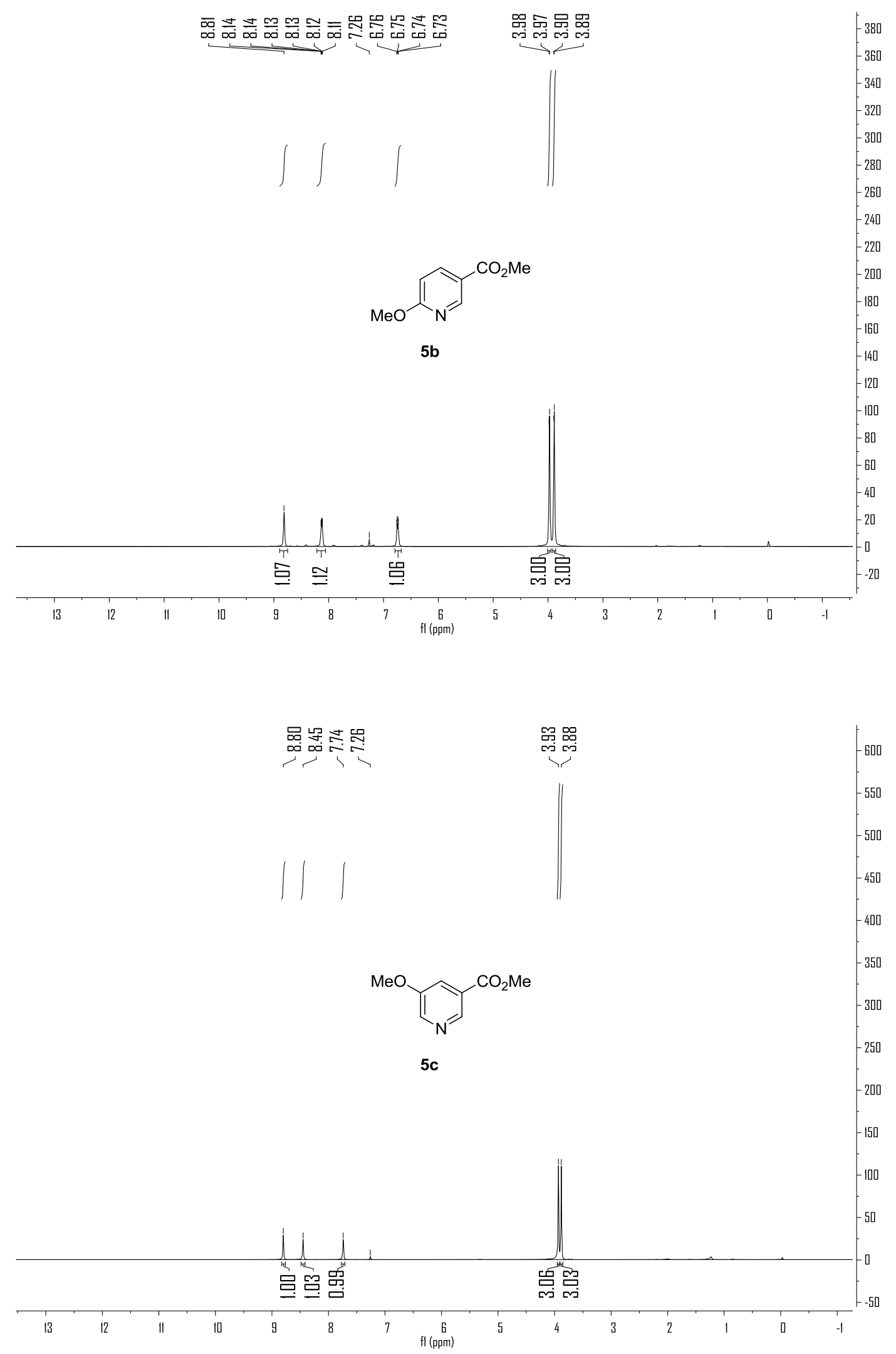

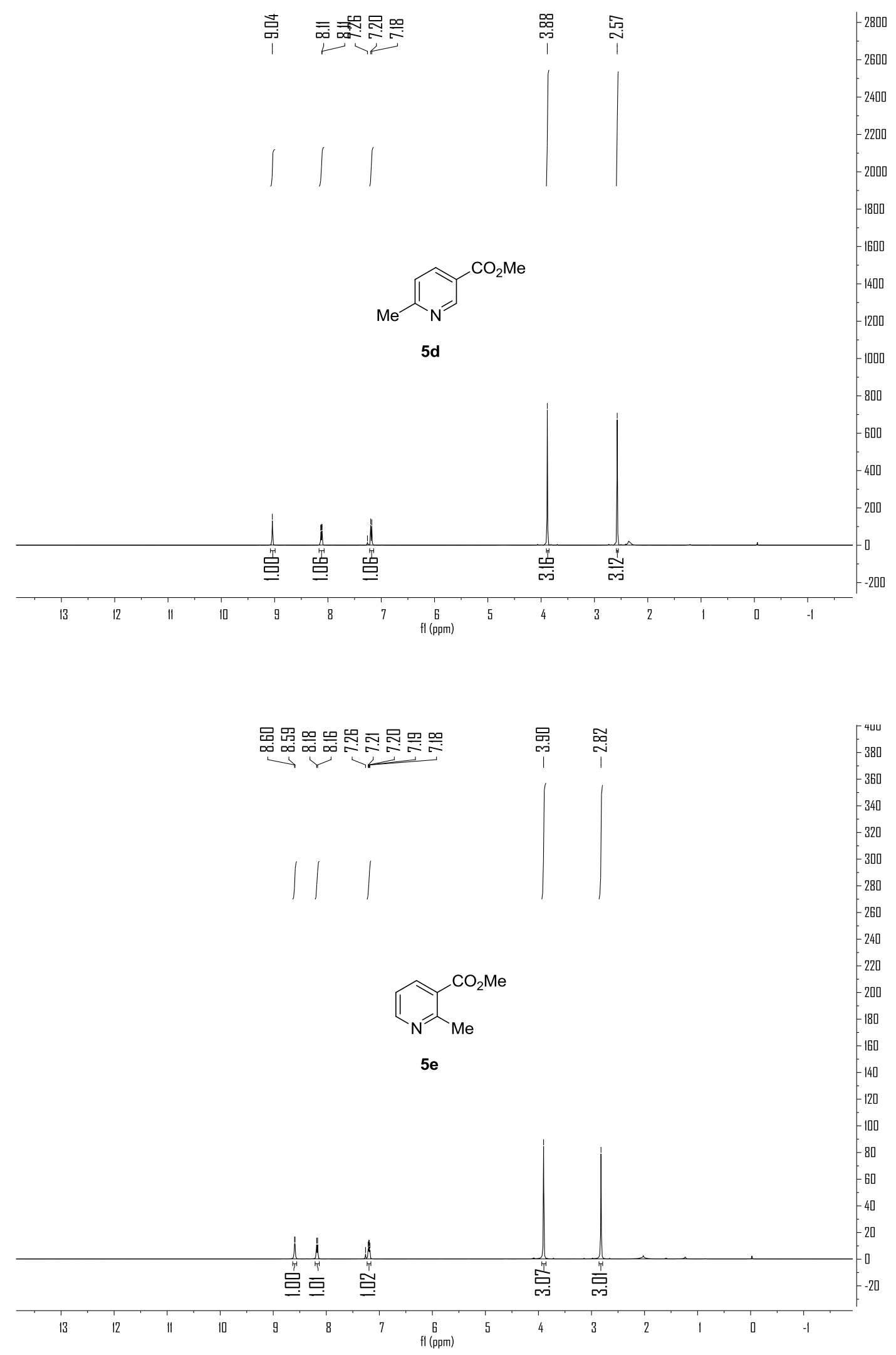

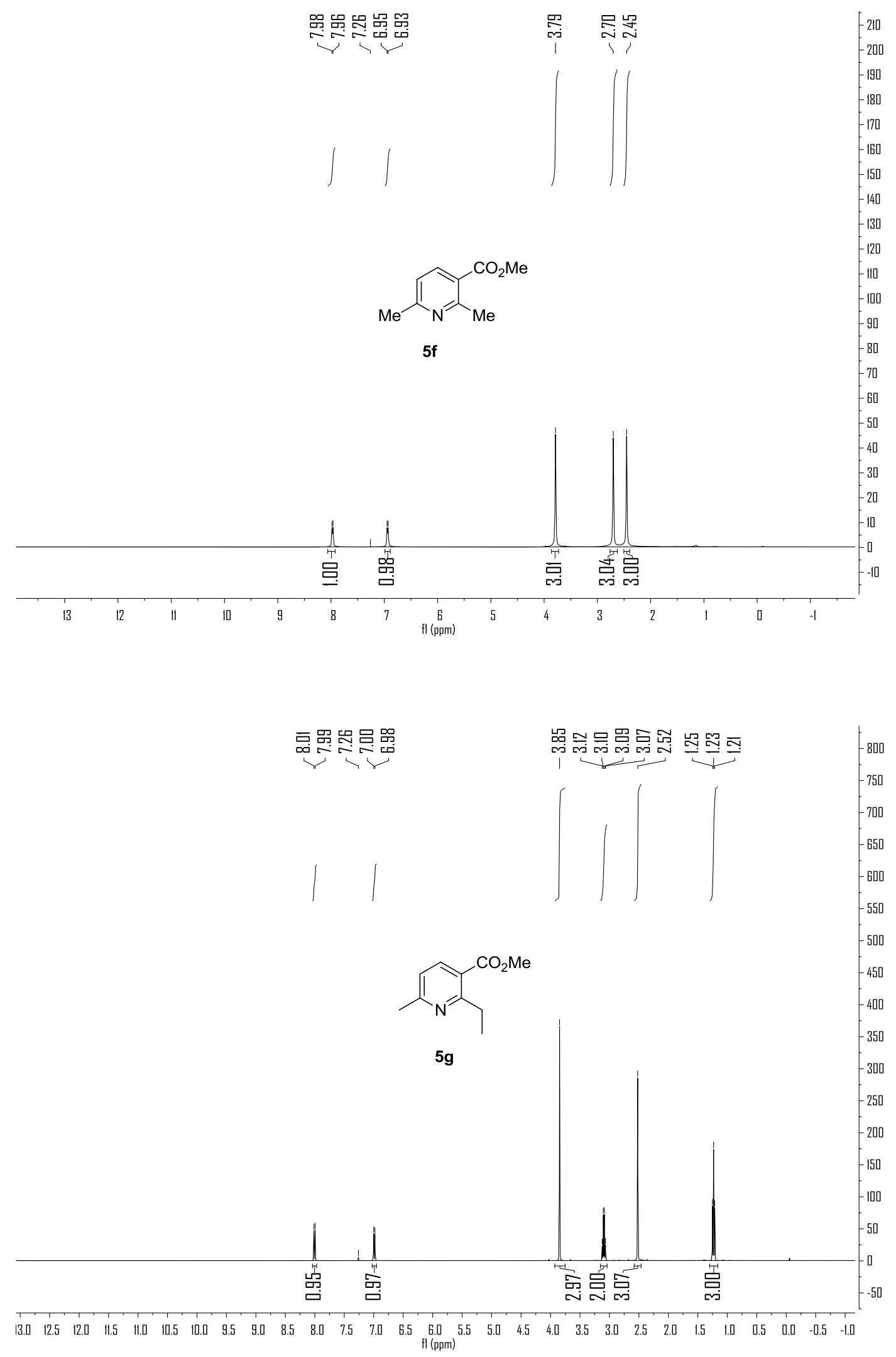

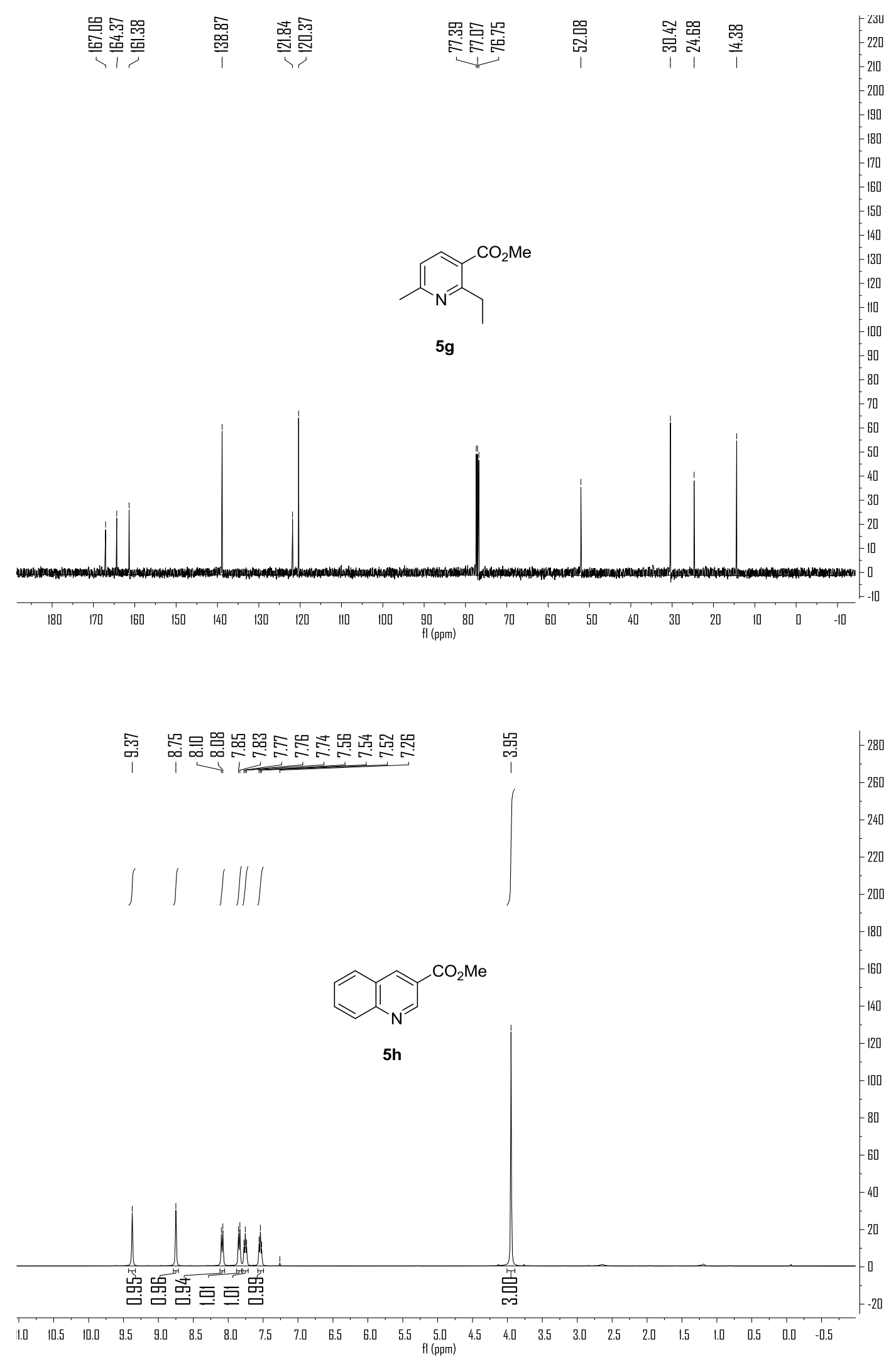

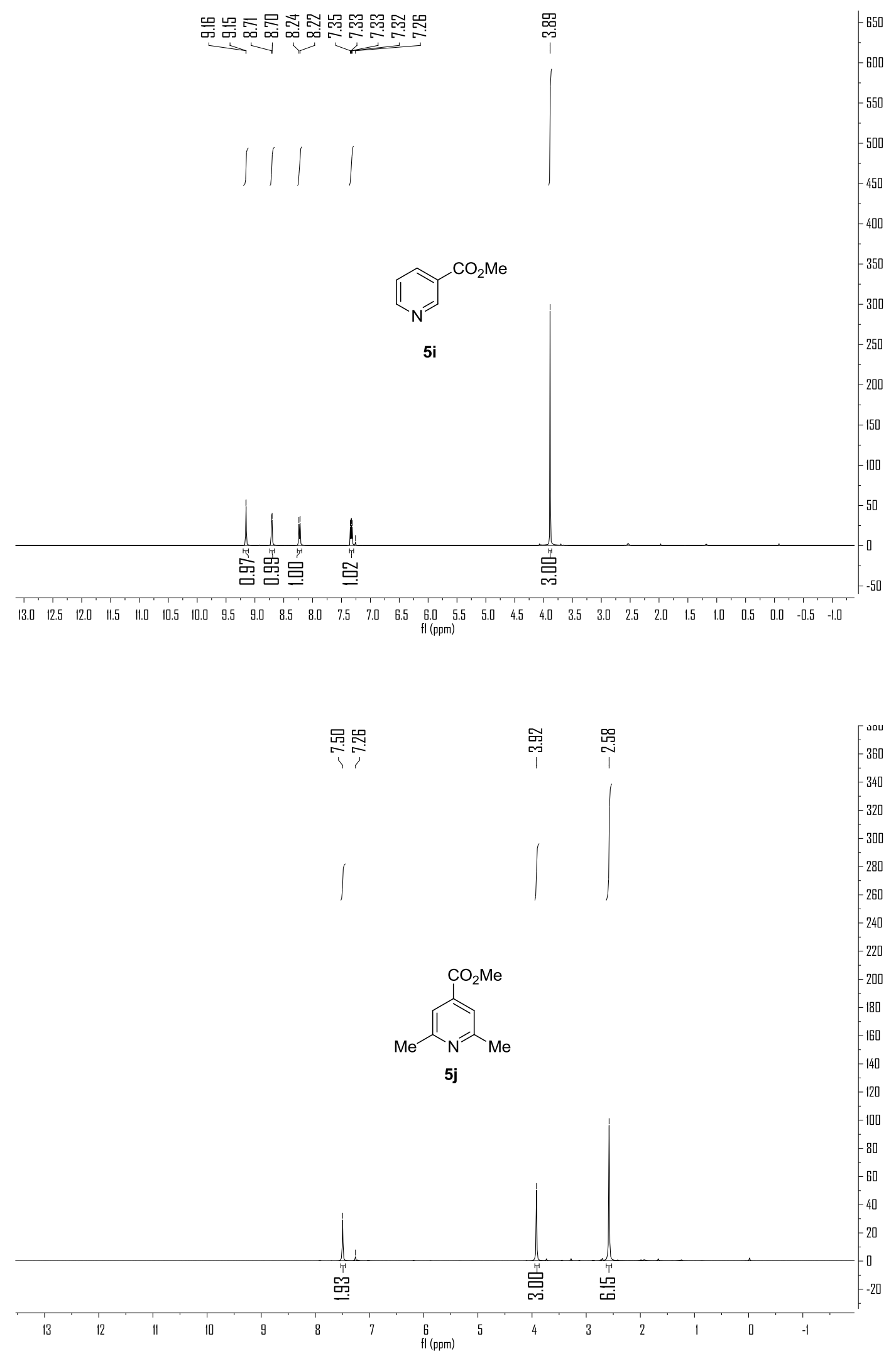

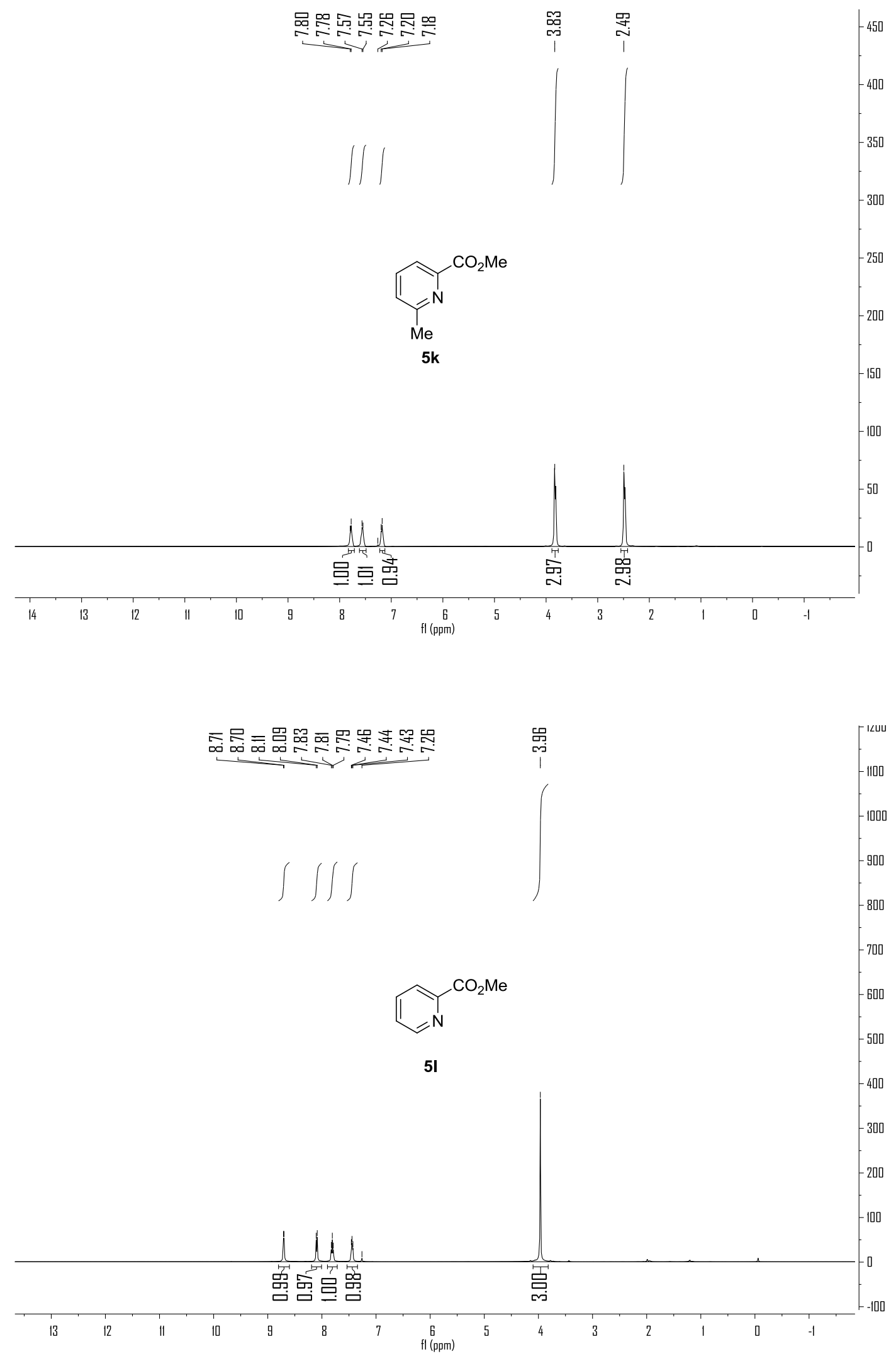